\title{
Contrast-enhanced spectral mammography in clinical practice
}

Citation for published version (APA):

Lalji, U. (2016). Contrast-enhanced spectral mammography in clinical practice: results in a screening population. [Doctoral Thesis, Maastricht University]. https://doi.org/10.26481/dis.20160512ul

Document status and date:

Published: 01/01/2016

DOI:

10.26481/dis.20160512ul

Document Version:

Publisher's PDF, also known as Version of record

\section{Please check the document version of this publication:}

- A submitted manuscript is the version of the article upon submission and before peer-review. There can be important differences between the submitted version and the official published version of record.

People interested in the research are advised to contact the author for the final version of the publication, or visit the DOI to the publisher's website.

- The final author version and the galley proof are versions of the publication after peer review.

- The final published version features the final layout of the paper including the volume, issue and page numbers.

Link to publication

\footnotetext{
General rights rights.

- You may freely distribute the URL identifying the publication in the public portal. please follow below link for the End User Agreement:

www.umlib.nl/taverne-license

Take down policy

If you believe that this document breaches copyright please contact us at:

repository@maastrichtuniversity.nl

providing details and we will investigate your claim.
}

Copyright and moral rights for the publications made accessible in the public portal are retained by the authors and/or other copyright owners and it is a condition of accessing publications that users recognise and abide by the legal requirements associated with these

- Users may download and print one copy of any publication from the public portal for the purpose of private study or research.

- You may not further distribute the material or use it for any profit-making activity or commercial gain

If the publication is distributed under the terms of Article $25 \mathrm{fa}$ of the Dutch Copyright Act, indicated by the "Taverne" license above, 


\section{Contrast enhanced spectral mammography in clinical practice:}

results in a screening population 
(C) Copyright Ulrich C. Lalji, Maastricht 2016

Cover design UlrichC. Lalji

Production \& print Datawyse | Universitaire Pers Maastricht

The printing of this thesis was financially supported by:

Angiocare B.V. The Netherlands | Bayer B.V. The Netherlands | C.R. Bard The Netherlands 


\title{
Contrast-enhanced spectral mammography in clinical practice: \\ results in a screening population
}

\author{
Proefschrift \\ Ter verkrijging van de graad van doctor aan de Universiteit Maastricht, op het \\ gezag van de Rector Magnificus prof. dr. L.L.G. Soete \\ volgens het besluit van het College van Decanen, \\ in het openbaar te verdedigen \\ op donderdag 12 mei 2016 om 16.00 uur \\ door \\ Ulrich Carlos Lalji
}

Geboren 18 september 1972 te Paramaribo 
Promotores

Prof. dr. J.E. Wildberger

Prof. dr. R.G.H. Beets-Tan

Copromotor

dr. M.B.I. Lobbes

Beoordelingscommissie:

Prof. dr. M.F. von Meyenfeldt (voorzitter)

Prof. dr. W.H. Backes

Prof. dr. C. Van Ongeval

Prof. dr. V.C.G. Tjan-Heijnen

Dr. J. Veltman 


\section{Content}

Chapter 1 Introduction

Chapter 2 Contrast-enhanced dual-energy mammography: a promising new imaging tool in breast cancer detection

Chapter 3 Radiation exposure of contrast-enhanced spectral mammography compared to full field digital mammography

Chapter 4 Evaluation of low-energy contrast-enhanced spectral mammography images by comparing them to full-field digital mammography using EUREF image quality criteria

Chapter 5 Contrast-enhanced spectral mammography in patients referred from the breast cancer screening program

Chapter 6 Contrast-enhanced spectral mammography in recalls from the dutch breast cancer screening program: validation of results in a large multireader, multicase study

Chapter 7 The quality of tumour size assessment by contrast-enhanced spectral mammography and the benefit of additional breast MRI

Chapter 8 Discussion

Summary

Samenvatting

Valorisation

Dankwoord

Biography

List of publications 



\section{Introduction}





\section{Introduction}

Breast cancer is the most common cancer in women [1]. According to the World Health Organization (WHO) an estimated 522.000 women died of breast cancer in 2012 [1]. Early detection of breast cancer is important to improve patient outcome and survival. In 2013, the invasive breast cancer incidence in the Netherlands was estimated at 169/100.000 women, whereas 3161 women died of breast cancer [2]. Since 1990 the number of invasive breast cancers has increased (Figure 1). Despite this fact survival has also increased. In the Netherlands the 5-year-survival of patients diagnosed with invasive breast cancer in the period from 2008 to 2012 was estimated to be $87 \%$, showing a steady increase in survival (Table 1) [2]. There are many factors that contribute to the increased survival rates.

Incidence of invasive breast cancer

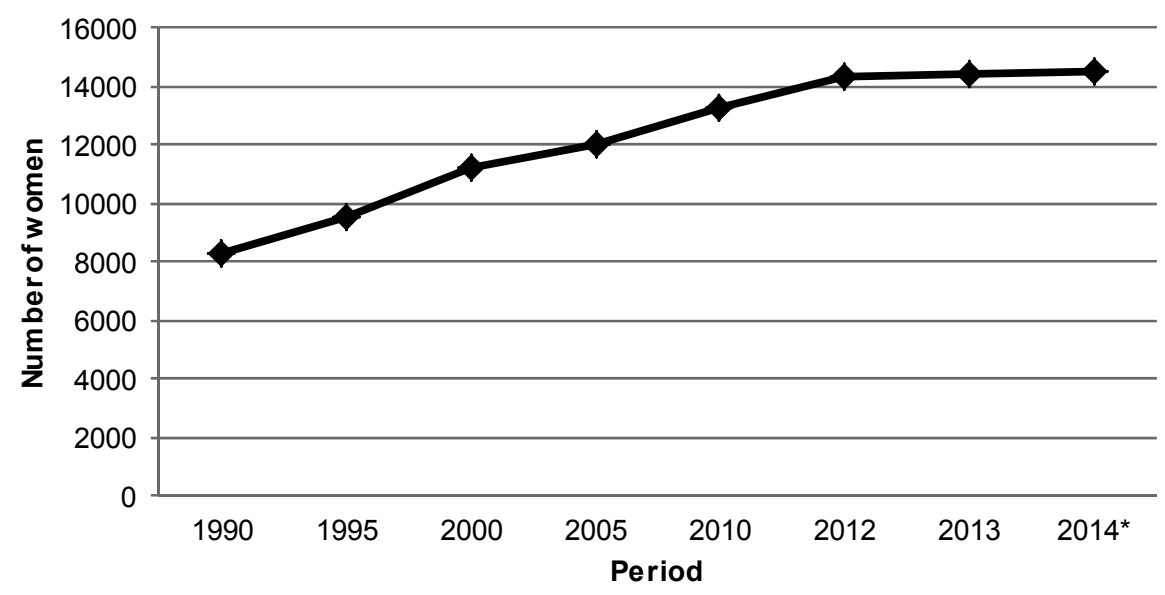

Figure 1: Incidence of invasive breast cancers in the Netherlands (2014* preliminary data). Source: Nederlandse Kankerregistratie, beheerd door IKNL (C) [march] 2015

The addition of radiotherapy to breast conserving surgery and drugs like the monoclonal antibody trastuzumab with or without a combination of chemotherapy contribute to the reduction in breast cancer mortality [3-5]. Additionally, screening programs also seem to reduce mortality rates as they are able to detect these invasive cancers at an early stage resulting in a more favourable prognosis [6]. In the Netherlands the national breast screening program was introduced in several regions in 1989. Starting from 1996, all Dutch women in the age group of 50 to 70 years are invited to participate. In 1997, the upper age limit was extended to 75 years.

At present, $80 \%$ of invited women participate in the screening program, which has a recall rate of $2.1 \%$. During screening mammography, standard mediolateral oblique (MLO) and craniocaudal (CC) views are obtained, with additional views 
performed when deemed necessary by the screening radiographers. These images are viewed by two independent radiologists who are blinded for each other's BIRADS score. If both radiologists detect a breast abnormality the patient is recalled. In case of discrepancies, a third (senior) radiologist is consulted for the final decision. This approach has been proven to be more sensitive than a non-blinded method [7]. Recalled women are referred to a hospital of their choice to undergo further evaluation of the detected abnormality. Breast cancer screening programs reduce breast cancer mortality by $25-31 \%$ [8].

Table 1: Survival given in percentage in the years after diagnosis of invasive breast cancer in the Netherlands. Source: Nederlandse Kankerregistratie, beheerd door IKNL (C) [march] 2015.

\begin{tabular}{llllllllllllll}
\hline $\begin{array}{l}\text { Period of } \\
\text { diagnosis }\end{array}$ & $\begin{array}{l}\text { Number of } \\
\text { tumours }\end{array}$ & \multicolumn{4}{c}{ Number of years after diagnosis, survival in percentage } \\
\cline { 2 - 13 } & 0 & 1 & 2 & 3 & 4 & 5 & 6 & 7 & 8 & 9 & 10 \\
\hline $1989-1993$ & 43560 & 100 & 95 & 90 & 85 & 80 & 77 & 74 & 71 & 69 & 66 & 64 \\
$1994-1998$ & 49770 & 100 & 95 & 91 & 87 & 83 & 80 & 77 & 75 & 73 & 71 & 69 \\
$1999-2003$ & 57537 & 100 & 96 & 92 & 89 & 86 & 84 & 82 & 80 & 78 & 77 & 75 \\
$2004-2007$ & 49588 & 100 & 97 & 94 & 91 & 88 & 86 & 83 & 81 & 80 & 78 & 77 \\
$2008-2012$ & 68127 & 100 & 97 & 94 & 92 & 89 & 87 & & & & & \\
\hline
\end{tabular}

Mammography continues to play an important role in breast cancer detection. Even though the development of digital mammography improved sensitivity and specificity, its diagnostic performance still depends heavily on breast density [9]. To overcome these limitations, new techniques are continuously being developed that were not feasible in the analogue era, for example the use of iodinated contrast agents.

Two approaches in contrast-enhanced mammography have been studied in the past years: temporal contrast-enhanced mammography (TCEM) and contrastenhanced spectral mammography (CESM, or contrast-enhanced dual energy mammography: CEDM) [10]. Both approaches showed promising pilot study results for breast cancer detection. However, TCEM suffered from some important limitations that hindered clinical implementation: one breast could be imaged in one direction, only. Other directions or the opposite breast would therefore require a new contrast bolus. Total acquisition time was approximately ten minutes, while the patient's breast remains compressed and patients should not move during this time to prevent motion artefacts. Contrast-enhanced Dual-Energy mammography (CEDM) or contrast-enhanced dual energy mammography (CESM) did not suffer from these limitations. In CESM, both breasts can be examined in two projection views, while the breast compression is released between image acquisitions. Consequently, CESM could potentially be a more practical solution for the daily clinical practice than TCEM. The first commercially available machines were installed in March 2011. 
In this thesis we aim to evaluate the clinical use and implementation of CESM. Therefore, we focused on several important items that can be encountered when a new imaging modality is introduced.

In chapter 2, a review of the principle of CESM is presented, together with a summary of the imaging protocol and patient handling during the examination. In addition, an overview of CESM's preliminary results and its potential future indications are discussed.

In chapter 3 we assessed the radiation dose of a standard CESM exam by comparing it to Full Field Digital Mammography (FFDM). Radiation exposure related data for both CESM and FFDM were studied after first validating the unit's output parameters using phantom experiments.

In chapter 4 we studied the image quality of the (low energy) CESM images by comparing them to FFDM images using well-defined image quality criteria as stated by the European Reference Organization for Quality Assured Breast Screening and Diagnostic Services (EUREF) [11].

In chapter 5 we evaluated the diagnostic accuracy of CESM in the first 113 women who were referred from the Dutch breast cancer screening program by comparing BI-RADS scores given to the exams by two radiologists experienced in reading CESM images. In this chapter, we aimed to evaluate the clinical feasibility and accuracy of CESM, even in a patient population with relatively low breast cancer prevalence.

We validated the initial results in chapter 6 in a study population of 199 new cases using a panel of ten readers with varying experience in reading CESM exams.

If the estimated tumour size measured on CESM and breast MRI is comparable then (hypothetically) pre-operative breast MRI might be omitted to assess breast cancer extent. Therefore, we studied the accuracy of tumour size estimation in both CESM and breast MRI exams in chapter 7 .

Finally, the study findings of this thesis are discussed in chapter 8 and an outlook on future perspectives of CESM are provided. 


\section{References:}

1. Ferlay J, Soerjomataram I, Ervik M, Dikshit R, Eser S, Mathers C, Rebelo M, Parkin DM, Forman D, Bray F GLOBOCAN 2012 v1.0, Cancer Incidence and Mortality Worldwide: IARC CancerBase No. 11 Lyon, France: International Agency for Research on Cancer; 2013. Available from: http://globocan.iarc.fr/Pages/fact_sheets_cancer.aspx. Accessed 7 Mar 2015

2. Nederlandse Kankerregistratie IC [march] 2015 Incidentie borstkanker. http://www.cijfersoverkanker.nl/selecties/Incidentie_borstkanker/img54d0ec5956814. Accessed 7 Mar 2015

3. Perez EA, Romond EH, Suman VJ, et al. (2014) Trastuzumab Plus Adjuvant Chemotherapy for Human Epidermal Growth Factor Receptor 2-Positive Breast Cancer: Planned Joint Analysis of Overall Survival From NSABP B-31 and NCCTG N9831. J Clin Oncol 32:3744-3752. doi: 10.1200/JCO.2014.55.5730

4. Moja L, Tagliabue L, Balduzzi S, et al. (2012) Trastuzumab containing regimens for early breast cancer. Cochrane database Syst Rev 4:CD006243. doi: 10.1002/14651858.CD006243.pub2

5. Darby S, McGale P, Correa C, et al. (2011) Effect of radiotherapy after breast-conserving surgery on 10-year recurrence and 15-year breast cancer death: meta-analysis of individual patient data for 10,801 women in 17 randomised trials. Lancet 378:1707-16. doi: 10.1016/S0140-6736(11)61629-2

6. Verbeek ALM, Broeders MJM, Otto SJ, et al. (2013) [Effects of the population screening into breast cancer]. Ned Tijdschr Geneeskd 157:A5218.

7. Klompenhouwer EG, Voogd AC, den Heeten GJ, et al. (2015) Blinded double reading yields a higher programme sensitivity than non-blinded double reading at digital screening mammography: A prospected population based study in the south of The Netherlands. Eur J Cancer 51:391-9. doi: 10.1016/j.ejca.2014.12.008

8. Broeders M, Moss S, Nyström L, et al. (2012) The impact of mammographic screening on breast cancer mortality in Europe: a review of observational studies. J Med Screen 19 Suppl 1:14-25. doi: 10.1258/jms.2012.012078

9. Carney PA, Miglioretti DL, Yankaskas BC, et al. (2003) Individual and combined effects of age, breast density, and hormone replacement therapy use on the accuracy of screening mammography. Ann Intern Med 138:168-75.

10. Lobbes MBI, Smidt ML, Houwers J, et al. (2013) Contrast-enhanced mammography: techniques, current results, and potential indications. Clin Radiol 68:935-44. doi: 10.1016/j.crad.2013.04.009

11. (2013) European guidelines for Quality Assurance in breast cancer screening and diagnosis, Fourth edition Supplements. Available from http://euref.org/european-guidelines 


\section{Contrast-enhanced dual-energy mammography: a promising new imaging tool in breast cancer detection}




\begin{abstract}
Contrast-enhanced Dual-Energy mammography (CEDM) is a promising new breast imaging tool for breast cancer detection. In CEDM, an iodine-based contrast agent is intravenously administered and subsequently, dual-energy mammography is performed. This results in a set of images containing both a regular mammogram and an image that contains contrast enhancement information. Preliminary studies have indicated that CEDM is superior to conventional mammography and might even match the diagnostic performance of breast MRI. In this review, the imaging technique, protocol and patient handling of CEDM is presented. Furthermore, an overview of current results on CEDM and potential future indications are outlined.
\end{abstract}




\section{Introduction}

Breast cancer is an important cancer-related cause of death in women[1]. In breast cancer, mammography plays a pivotal role in cancer detection and follow up, despite the availability of various other breast imaging modalities, such as ultrasound and breast MRI.

One of the most important limitations of mammography is that its diagnostic performance depends heavily on breast density [2]. To overcome this limitation, new techniques are being developed that were not feasible in the analogue era. One of these techniques is the use of iodinated contrast agents in (contrastenhanced) mammography. Several approaches in contrast-enhanced mammography have been studied in the past years [3], and all of these showed promising feasibility results for breast cancer detection. Of these various techniques, Contrast-enhanced Dual-Energy mammography (CEDM) or Contrast-enhanced Spectral mammography (CESM) became commercially available some years ago and seems to be the most promising approach for routine clinical use.

The use of contrast agents in cancer detection is based on the phenomenon that neoplasms induce angiogenesis for further tumour growth. However, these new vessels are rapidly formed and as a result, they are 'leaky'. Contrast agents can pass through these vessel walls into the (tumour) interstitium, causing enhancement. For breast cancer, this principle is well-known in breast MRI and has helped to increase sensitivity and specificity for breast cancer detection [4].

In this review, the imaging technique, protocol and patient handling of CEDM is presented. Furthermore, an overview of current results on CEDM and potential future indications are outlined.

\section{Imaging protocol}

\section{CEDM image acquisition}

In CEDM, two images are obtained per breast: a low energy and a high energy image. The low-energy image is acquired by using a molybdenum (Mo) and rhodium (Rh) target and Mo and Rh filters at a peak kilo voltage in the range of 26 to 31 $\mathrm{kVp}$. Since the k-edge of iodine is $33.2 \mathrm{KeV}$, the used spectrum is below this point. Consequently, the low-energy images provide maximal soft tissue contrast and are in fact comparable to a regular mammogram. Subsequently, the high-energy image is acquired by using a Mo target and double layer filter of aluminium (Al) and copper $(\mathrm{Cu})$ with a peak kilo voltage ranging from 45 to $49 \mathrm{kVp}$. Since this spectrum is above the k-edge of iodine, the obtained image contains information on iodine presence (enhancement). The high-energy image is not diagnostic and is used for post-processing purposes. In this post-processing step, these two images are combined to create a 'recombined' image that displays areas of contrast enhancement (Figure 1). 
In contrast to previous suggested contrast-enhanced mammography techniques, CEDM is able to image both breasts in mediolateral (MLO) and craniocaudal (CC) views with a single contrast administration. The additional highenergy image is acquired in an additional 4 seconds and breast compression is relieved between views. Although dependent on breast thickness, the estimated combined dose from the low and high energy images is approximately 1.2 times the dose delivered in a standard single-view digital mammography [5]
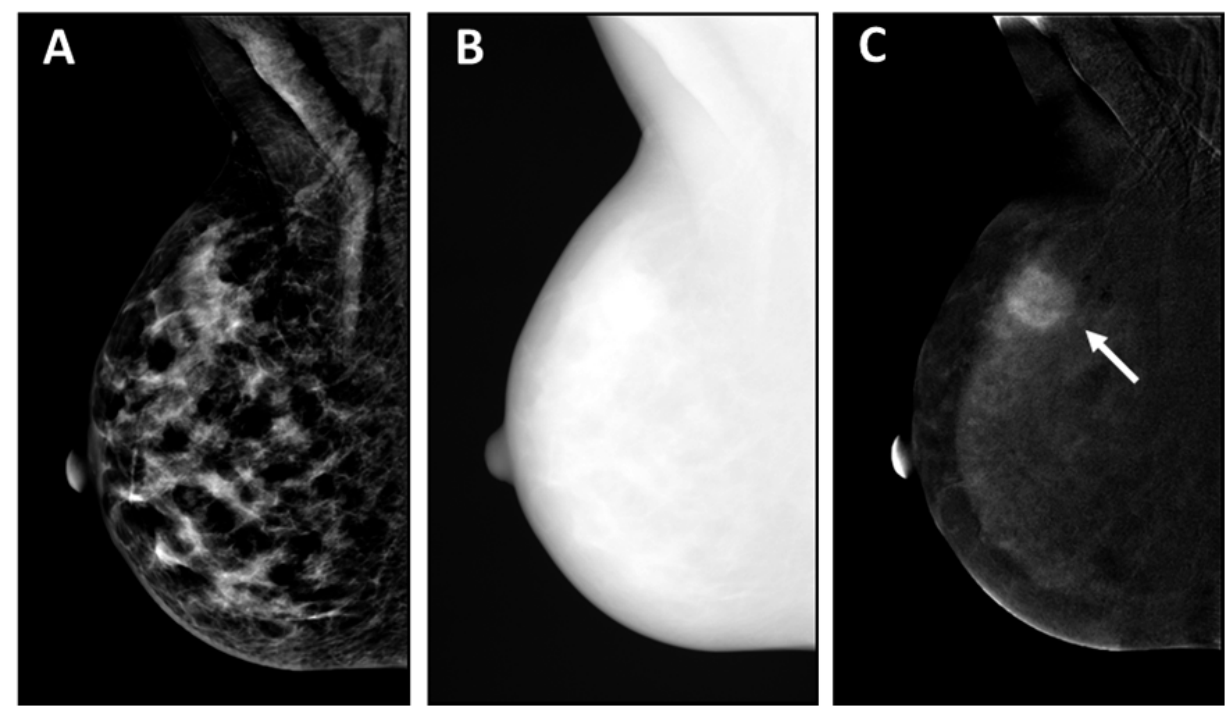

Figure 1: Example of CEDM (only right MLO view shown). In CESM, a low-energy image (A) is first acquired, which is comparable to a regular mammogram. Subsequently, a high-energy image (B) is acquired, which is not diagnostic, but which is used to generate a recombined image (C), which clearly shows an ill-defined enhancing mass in the upper part of the breast (arrow). Histopathological analysis revealed an invasive ductal carcinoma

\section{Patient handling}

In our institution, patients undergoing CEDM receive $1.5 \mathrm{~mL}$ iodinated contrast per kilogram of body weight intravenously, using an automated power injector at a rate of $3 \mathrm{~mL}$ per second, followed by a saline flush. The optimal dose and concentration of iodine contrast agent is unknown, and the current protocol is based on experiences in pilot studies. However, it is to be expected that the imaging protocol can be optimized further, not only regarding contrast dose, but also its timing. Future comparative studies are needed to further optimize the imaging protocol. Nonetheless, the current protocol enables CEDM users to easily reproduce findings of previous studies without risk of comprising diagnostic accuracy. Two minutes after contrast administration, a CC view of the breast without a suspicious abnormality is acquired, followed by a CC and MLO view of the other breast, followed by a MLO view of the first breast. Total imaging time is approximately 7 minutes; the 
total time of examination (including contrast injection) is approximately 20 minutes. At the end of the exam, patients are monitored for an additional 15 minutes to rule out adverse contrast reactions.

Contra-indications for the intravenous administration of an iodine-based contrast agent have to be considered before any CEDM exam, such as (prior) allergic reaction to iodine-based contrast agents or known renal function impairment. The current generation of iodine-based contrast agents is well tolerated and considered safe. In patients that receive a low-osmolality agent adverse reactions can occur in 1 to $3 \%$ [6]. The majority of these reactions are mild and often selflimiting. In some events, intravenous medication can result in resolvement of the adverse reaction. However, more severe reactions occur in approximately 0.2 to $0.7 \%$ of the cases, and may consist of bronchospasms, hypotension or hypertension, laryngeal oedema, tachycardia or bradycardia, seizures, or pulmonary oedema [7][8]. Fatalities as a result of contrast administration are considered to be rare [9].

Any administration of an iodine-based contrast agent could result in contrastinduced nephropathy (CIN), which is defined as 'a condition in which an impairment of renal function (i.e. an increase of serum creatinine by more than $25 \%$ or $44 \mu \mathrm{mol} / \mathrm{L}$ occurs within three days following intravascular administration of contrast medium in the absence of an alternative aetiology' [10]. To assess the risk of developing CIN, the Contrast Media Safety Committee of the European Society of Urology provided several guidelines for the safe use of contrast agents, which were recently updated [10]. With aid of these guidelines, patients who are at risk of developing CIN can be identified using a questionnaire. In patients at risk according to this questionnaire should have their renal function checked by a blood sample prior to the examination. If necessary, precautionary prehydration and monitoring of renal function should be considered. According to the ESUR guidelines [10] an eGFR $<45 \mathrm{ml} / \mathrm{min} / 1.73 \mathrm{~m} 2$ is considered a risk factor in developing CIN.

There is a large variation regarding the policy on preparative fasting before the administration of an iodine-based contrast agent. Although many institutions still have a protocol on fasting before these exams, Lee et al. showed that there is no evidence that fasting prior an examination results in a significant reduction of aspiration-related complications[11]. Consequently, preparative fasting prior to CEDM is not necessary.

\section{Current results of CEDM}

In 2003, Lewin et al. published a feasibility study on CEDM[12] . In this study, 13 invasive breast cancers in 26 patients were studied using this technique. Unlike current CEDM protocols, their protocol consisted of the imaging of a single breast 
in a single view. Eleven breast cancers showed strong enhancement after contrast administration. One breast cancer showed moderate enhancement, and one weak enhancement. In benign cases, they observed diffuse enhancement in two cases, and weak focal enhancement in two others. Despite a very small study population, their pilot results warranted further research. A major limitation of their protocol was that the exam consisted of one breast in a single view. With this approach, the contralateral breast could not be evaluated, and ultrasound-guided localization of the abnormality might be difficult in single view exams.

The next step in the development of CEDM consisted of contrast-enhanced, dual energy mammography of a single breast in a single view, but at different time points. The aim of this approach was to investigate signal intensity time curves of various breast lesions, similar to the curves known from breast MRI [13]. Diekmann et al. studied 21 patients with 26 breast lesions (10 benign, 15 malignant, one borderline type of adenomyoepithelioma) [14]. In their study, all malignant lesions showed enhancement. One low-grade ductal carcinoma in situ failed to show enhancement. They demonstrated that half of the benign lesions did not show enhancement. However, their study failed to demonstrate any resemblance in enhancement kinetic analysis to breast MRI.

Dromain et al. studied 20 patients with a similar approach [15]. In their study, 16 out of 20 breast cancers showed enhancement. The malignant cases that did not show any enhancement were all invasive breast cancers. They observed a good correlation between tumour diameter assessed with contrast-enhanced mammography and histopathological specimens: r2=0.743. Unfortunately, no information on agreement was provided, and good correlation does not automatically imply good agreement [3]. In addition, a poor correlation was found between mammography results and intratumoural micro vessel density. A major limitation of this study was that due to the analysis of solely breast cancer cases, no information on specificity could be calculated.

Diekmann et al. published a second study on CEDM in 2011, in which 80 breast lesions in 70 patients were evaluated (50 benign, 30 malignant) [16]. In this study, multiple readers evaluated the images, and all readers detected more breast cancers in absolute terms using this technique. On average, 5.7 more malignant lesions were detected with CEDM, but they still used different image acquisitions at different time points to evaluate enhancement kinetics. Unfortunately, the kinetic analysis information showed a large variation and the authors concluded that kinetic analysis with this technique remained challenging.

The imaging protocol so far, which consisted of CEDM of a single breast in a single view, had some important limitations: (1) it did not provide any information on the contralateral breast, while it is known that contralateral breast cancer can occur in $3 \%$ of the cases [17]; (2) image acquisition is rather long, while the breast remains compressed, resulting in patient discomfort; (3) the lack of a second projection 
view hampers the detection of the lesion with additional imaging, for example targeted ultrasound for biopsy guidance. Also, the studies so far failed to reproduce the enhancement kinetics analysis that is so useful in breast MRI. As a result, the image protocol changed and the different time points were substituted for CEDM exams of both breasts in two views (CC and MLO)[18].

With this new approach, Dromain et al. studied 142 breast lesions (62 benign, 80 malignant). Sensitivity for CEDM (93\%) was significantly higher than conventional mammography (78\%), at no loss of specificity. All multifocal breast cancers were correctly identified by CEDM [19]. Shortly thereafter Dromain et al. published a second paper with largely the same study population, studying 148 breast lesions in a multireader study [5]. The clinical performance (as expressed by the area under the receiver operating characteristic curve) increased for all readers for CEDM and ultrasound (0.87) as compared to conventional mammography and ultrasound (0.83).

Jochelson et al. compared CEDM to conventional mammography and breast MRI in 52 breast cancers [20]. Sensitivity was an equal 96\% for both CEDM and breast MRI, and superior to conventional mammography: 81\%. CEDM resulted in less false-positive findings as compared to breast MRI, but breast MRI was more accurate in detecting multifocal breast cancers. However, their sample size was rather limited, but the results showed that CEDM has the potential to replace breast MRI for some indications.

Most recently, Fallenberg et al. showed in 80 patients that breast cancer detection improved in CEDM as compared to conventional mammography. In addition, tumour size measurement between CEDM and breast MRI were compared, showing that there was no significant difference between lesion measurements on MRI and CEDM compared with histopathology [21].In their study, Pearson's correlation coefficients for CEDM versus histopathology and MRI versus histopathology were 0.773 and 0.654 , respectively.

In summary, several studies confirmed that CEDM has promising potential as a breast imaging tool. Current studies show that CEDM is superior to regular mammography and might even match the diagnostic performance of breast MRI. The number of false-positive findings could even be less in CEDM compared to breast MRI, but this has not been studied extensively. A major limitation of the studies published so far is their limited number of included patients and populations having a rather high breast cancer prevalence, which does not reflect the population of a routine clinical practice. If, for example, CEDM would results in many falsepositive findings in populations with lower breast cancer prevalence, the technique would still be regarded as useless. Consequently, future studies are needed to evaluate the diagnostic performance of CEDM in populations with much lower disease prevalence. 


\section{Potential indications}

Because the principle of tumour enhancement used in CEDM and contrastenhanced breast MRI are similar, it is to be expected that CEDM indications could also be similar to those of breast MRI. Current accepted breast MRI indications are assessment of preoperative disease extent, response monitoring in patients receiving neoadjuvant chemotherapy, evaluation of the breast after breast-conservative therapy, inconclusive findings, screening of high risk patients, and unknown primary cancers[22]. Although image guided interventions and implant imaging are also accepted breast MRI indications, these indications are not (yet) feasible with CEDM.

\section{Preoperative assessment of disease extent}

In breast cancer detection and disease extent evaluation, CEDM has been shown to be superior when compared to mammography, and similar or even better than breast MRI (Figure 2) [20, 21]. Ductal carcinoma in situ (DCIS) is most commonly associated with the presence of micro calcifications. Although DCIS can be detected with breast MRI [13], low-grade or some intermediate-grade DCIS might not show enhancement and might be misinterpreted on breast MRI. Besides visualizing enhancing masses and lesions, CEDM has the added benefit to evaluate micro calcifications using the low dose images, which is not possible with MRI. Breast MRI is the most accurate technique to analyse disease extent [23], showing a good correlation with tumour extent assessed by pathology specimens. In a recent study, CEDM has shown to have an excellent correlation with respect to the evaluation of disease extent [21]. Although promising, current study results are still based on limited sample sizes. Nonetheless, they showed CEDM's potential to assess disease extent as well as breast MRI. Multifocality might be a problem, since Jochelson et al. showed that breast MRI remains superior to CEDM in the evaluation of multifocal tumours[20]. However, there were only very few cases of multifocal cancers in this population, and no scientific conclusions can be drawn from this study with respect to the assessment of multifocal breast cancers. More studies are needed to evaluate the potential of disease extent evaluation with CEDM, but CEDM might already provide a suitable alternative for assessing disease extent in patients with contra-indications for MRI or claustrophobia.

\section{Response monitoring of neoadjuvant chemotherapy}

In response monitoring of patients receiving neoadjuvant chemotherapy (NAC), breast MRI has shown to be the most accurate modality [24]. For this purpose, breast MRI sequences can be used for either early response monitoring (i.e. after several cycles of chemotherapy), or after completion of the therapy to assess the presence of residual disease[25, 26]. The accuracy of breast MRI in response moni- 
toring is dependent on breast cancer subtypes and therapy regimen and is currently a topic of extensive research.

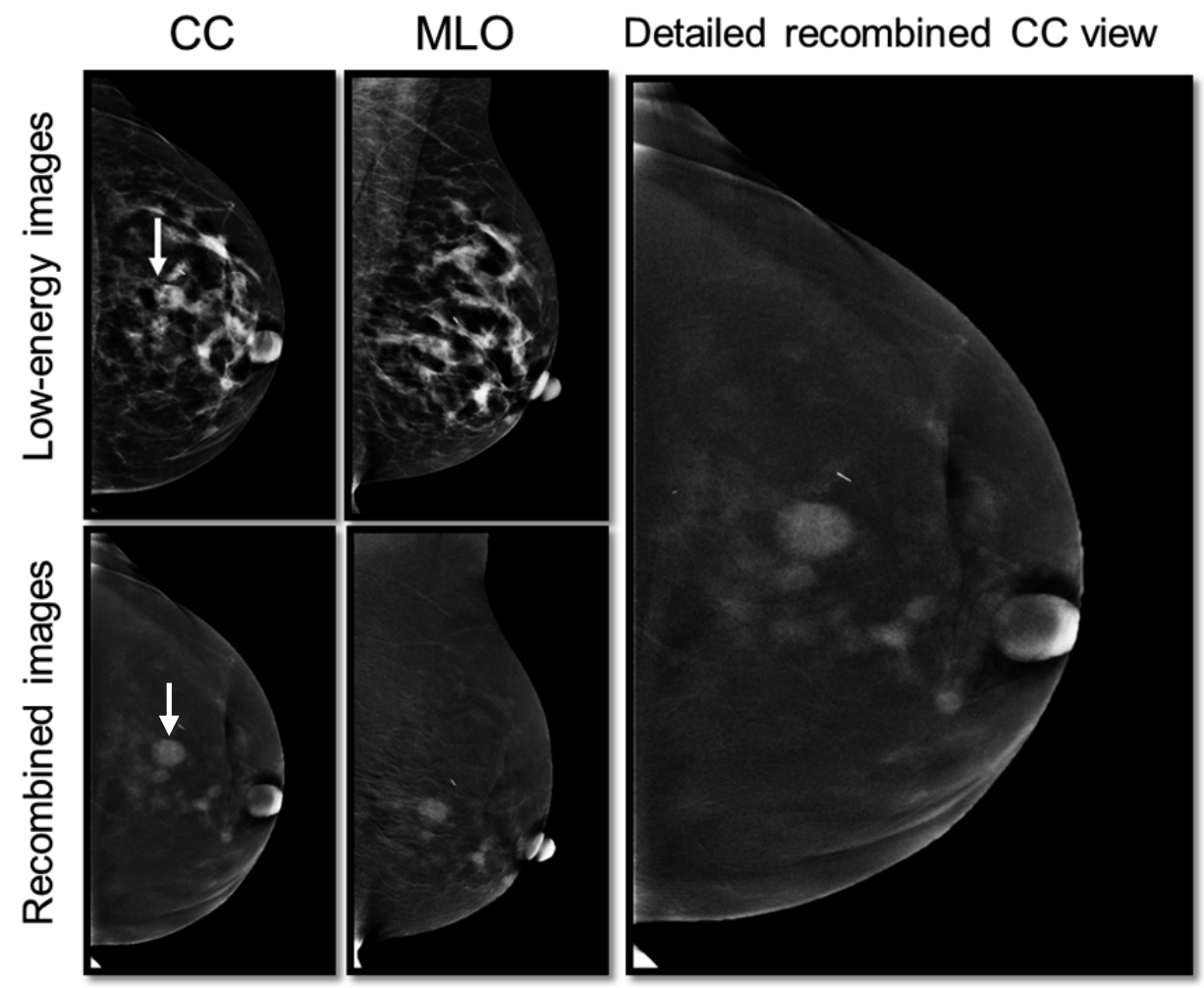

Figure 2: A round density was observed in the left craniocaudal (CC) view (arrow), not visible on the mediolateral oblique (MLO) view. CESM showed true disease extent, with multiple enhancing masses in multiple quadrants of the breast. Histopathology of the surgical specimen after mastectomy confirmed extensive multifocal, multicentric invasive ductal carcinoma

We were able to test the hypothesis that CEDM can assess response to NAC in several volunteers who underwent CEDM as part of their preoperative evaluation. In these proof-of-concept exams, we were able to demonstrate that it is feasible (Figure 3), and it is currently being studied in multiple centres.

\section{Breast evaluation after breast-conservative therapy}

In this setting, CEDM might be used for evaluation of residual disease after positive tumour margins, or as a method to detect local recurrence. Scar tissue is an important factor in every imaging modality that is used for evaluation after breastconservative therapy. The traction of the scar tissue itself results in irregular or speculated morphology, which makes early detection of local recurrence difficult. In our institution, we were able to detect one local recurrence with CEDM, showing an enhancing mass within the scar tissue. Even more important, it showed disease 
extent adequately despite the underlying distortions of the scar tissue (Figure 4). However, since these cases are rare due to improvements in breast cancer therapy, we need to collect more of them. From breast MRI experiences, it is known that detection of residual disease after recent surgery with tumour positive margins is hampered due to aspecific enhancement caused by healing processes. Due to the similarity in principle of enhancement in CEDM, similar limitations can be expected. However, no case reports or studies have published findings on these kinds of patients.

Baseline CESM
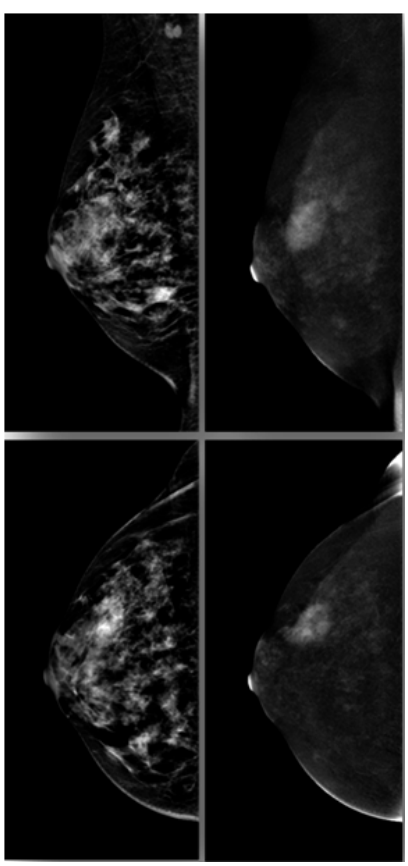

\section{CESM after NAC}
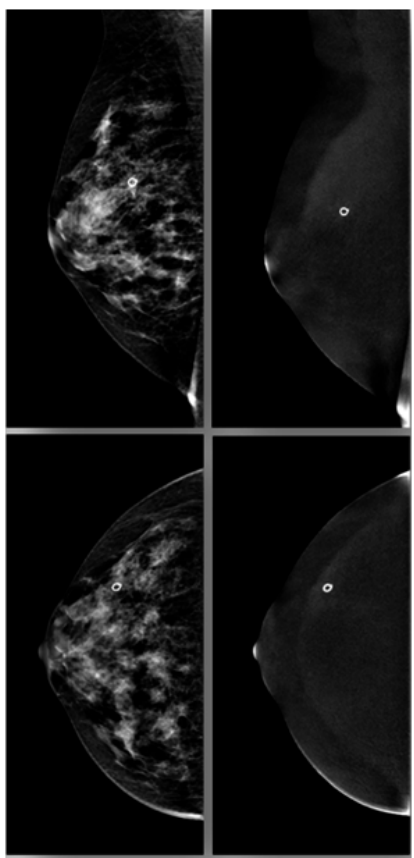
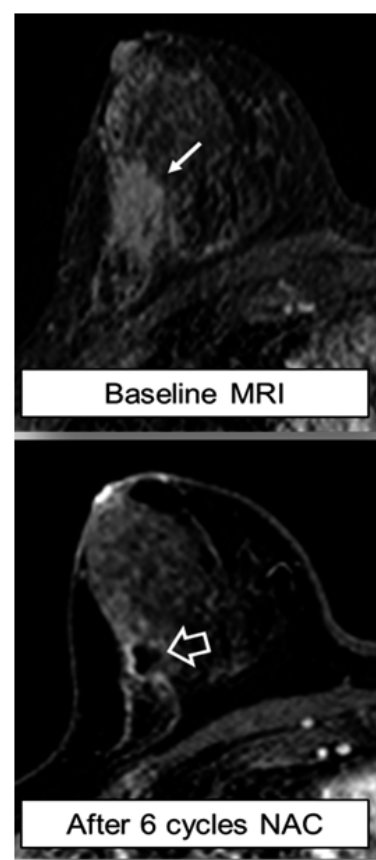

Figure 3: Neoadjuvant chemotherapy (NAC) response monitoring using CESM. An invasive breast cancer was diagnosed in the right breast and the patient was treated with NAC. After six cycles, complete response was suggested by CESM. At the prior tumour site (now marked) no enhancement was visible. Imaging findings were similar to breast MRI (right column) and confirmed by histopathologic examination of the surgical specimen (complete response, Miller and Payne grade 5).

\section{Screening of high risk patients}

For screening purposes, contrast administration and its potential side-effects will hamper the use of CEDM in a general population. Several patient populations with increased risk of developing breast cancer, such as BRCA 1 and 2 gene mutation carriers, Cowden and Li Fraumeni syndrome and their relatives, or women that underwent chest radiotherapy early in life have been shown to benefit from annual screening with breast MRI. In the Netherlands, these women receive annual breast MRI from the age of 25 , which is combined with annual mammography (to detect 
micro calcifications) from the age of $30[27,28]$. These women have to visit our department twice for these exams. However, these (young) women are more sensitive to the effect of ionizing radiation of their (dense) breasts, so dose will be an issue particularly in these women. Dose measurements reported so far were equal to conventional mammography [21], or slightly higher: $+20 \%$ [5]. To compensate for minor additional dose in CEDM, these women might be considered to only undergo CEDM in MLO views, with additional views performed only when indicated. However, current reports on CEDM dose were part of a sub analysis in a larger study, and no objective standardized dose measurements have been performed thus far. These are needed to clarify the additional dose that is associated with CEDM and should be weighed against the potential benefit of screening high risk women with CEDM.
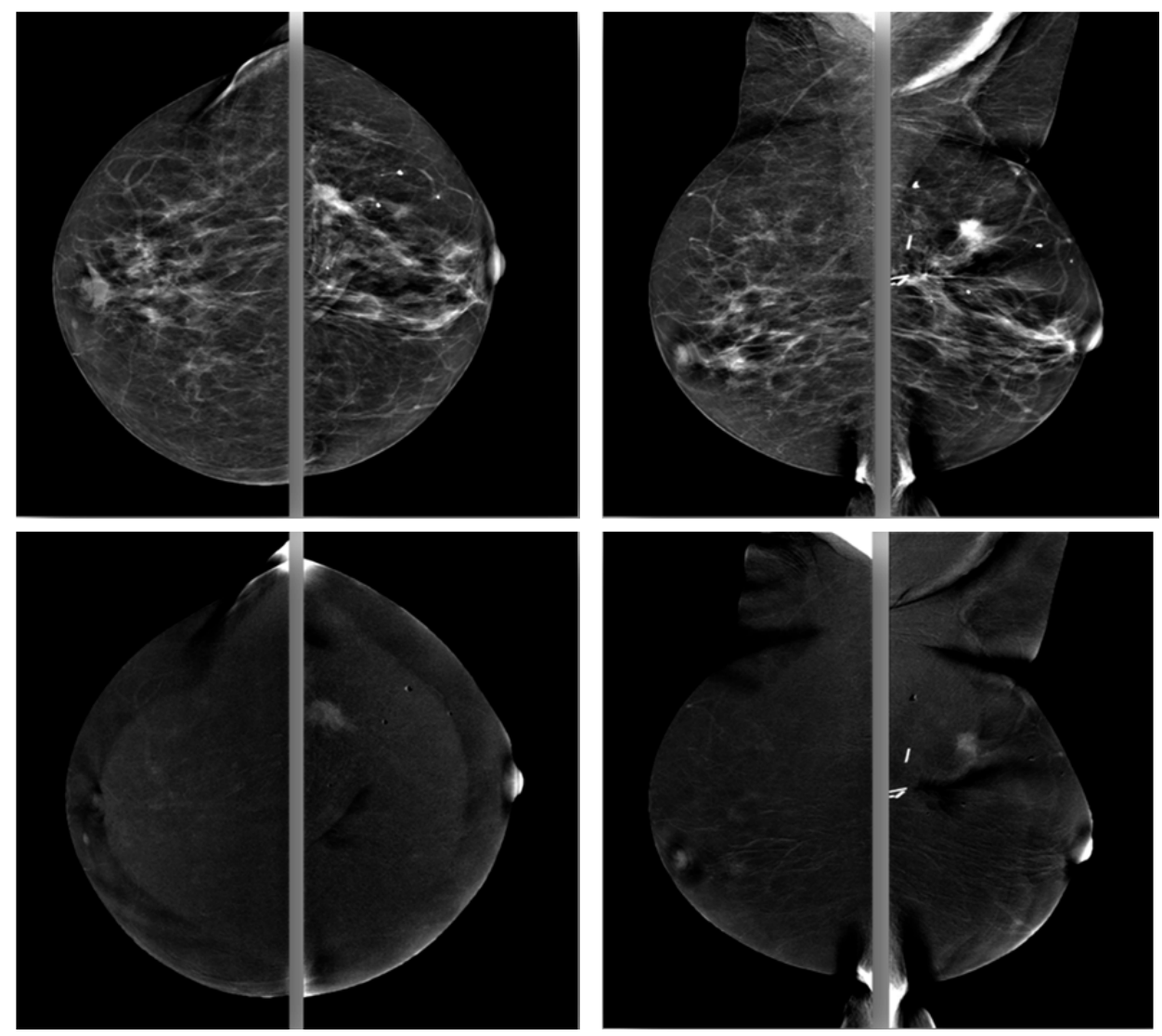

Figure 4: Local recurrence after previous breast-conservative surgery, showing a new nodular mass at the previous lumpectomy site in the left breast. Biopsy revealed invasive breast cancer 


\section{Inconclusive findings}

A frequently occurring indication for breast MRI is an inconclusive finding on conventional mammography. Because of the high negative predictive value of breast MRI, underlying breast cancer can be ruled out if breast MRI is negative. For MRI the positive predictive value and negative predicted value is reported to be $72 \%$ and $85 \%$ respectively [29]. In this study, patients with negative needle biopsies that did not yield specific benign diagnoses (e.g., fibroadenoma, papilloma) and who did not undergo subsequent excisional biopsy underwent clinical and mammographic follow-up after 1 year to ensure stability of the suspicious lesion

In our institution CEDM is used for the evaluation of women referred from breast cancer screening. Although some cancers are obvious on conventional mammography, the majority of these referrals can be considered as 'inconclusive findings'. Preliminary results of the first 80 women in this study also demonstrated a negative predictive value of CEDM of $100 \%$, suggesting that (similar to breast MRI) breast cancer could be virtually ruled out if CEDM is negative. However, this study is currently ongoing to include a larger number of patients.

\section{Unknown primary cancers}

In these patients, metastases elsewhere have been found, but a primary tumour site is still unidentified. It is known from breast MRI, that it is abnormal in $50 \%$ of the cases where a conventional mammogram was negative. These numbers even increase to $75-85 \%$ if the metastases were found in the axilla. Consequently, it is recommended to perform breast MRI in cases with unknown primary cancers when the initial mammogram was negative. However, this implies an additional exam in patients who already are undergoing extensive evaluation for this problem. Although no case examples have been reported so far to confirm the use of CEDM in these cases, it might be considered since principles behind CEDM and breast MRI are comparable.

\section{CEDM guided interventions and implant imaging}

CEDM guided interventions are not (yet) available, but are a topic of technical developments. Although most enhancing lesions on CEDM can to some extent be visualized on the low-energy image, some enhancing structures might remain occult even when compared to the low-energy images. It is mandatory that a 'CEDMguided stereotactic' biopsy becomes available when its technical issues are resolved. However, chances are very high that this structure will enhance on breast MRI too, making it accessible for MRI-guided biopsy. This latter approach is especially difficult for institutions with limited access to MRI and inability to perform breast MRI-guided biopsies themselves. Thus, a CEDM-guided biopsy technique is essential for CEDM to become a clinical success in many hospitals worldwide.

The integrity of breast implants cannot be assessed using CEDM. Breast MRI, using dedicated sequence protocols, remains essential for this evaluation. Also, 
CEDM is more challenging in breast implants, since exposure times will be increased due to implant presence. As a result, the X-ray tube will generate more heat and needs to cool down longer, hampering the CEDM imaging protocol.

\section{Conclusion}

In summary, CEDM is a potentially important imaging tool in detecting and diagnosing breast cancer. Several studies have already shown its superiority when compared to conventional mammography. In addition, recent studies have shown that its diagnostic performance might match that of the most accurate breast imaging modality so far: breast MRI. However, to further validate the strength and weaknesses of CEDM more studies are needed in order to define proper CEDM indications.

\section{Future perspective}

Although preliminary CEDM studies have shown promising results, the study sample sizes were limited and populations consisted of a high prevalence of breast cancer. This is suitable for feasibility studies, but it does not reflect true clinical populations, in which most women do not have breast cancer. Future studies should also evaluate the diagnostic performance of CEDM in populations with low disease prevalence. If CEDM would results in a high number of false-positive findings, the technique would still be useless in everyday clinical practices.

An interesting development in the area of contrast-enhanced mammography is Contrast-enhanced Dual-Energy Tomosynthesis(CEDT). In tomosynthesis, the compressed breast is imaged in multiple planes, resulting in three dimensional imaging volumes through which the user can scroll, usually in $1 \mathrm{~mm}$ slices[30]. Several prototype machines are currently under investigation. Recently, Froeling et al. demonstrated that with this technique, signal intensity time curves might be evaluated with mammography, similar to breast MRI. In their study, they showed a significant correlation between contrast kinetics in the signal intensity time curves between CEDT and breast MRI, which might further aid in breast lesion evaluation [31].

Finally, since the principle of tumour enhancement are similar in CEDM and breast MRI, similar indications might also be expected. Preliminary studies have shown CEDM's potential as a replacement for breast MRI in preoperative evaluation of disease extent and its ability to solve inconclusive findings on conventional imaging, but the number of cases studied is still rather limited. In addition, other potential indications, such as response monitoring after NAC, evaluation of the breast after conservative therapy, screening of high risk patients, and evaluation of patients with unknown primary malignancies are topics of ongoing studies, but the proof of principle has been shown in pilot data and case examples. 


\section{References}

1. Siegel R, Naishadham D, Jemal A (2013) Cancer statistics, 2013. CA Cancer J Clin 63:11-30. doi: 10.3322/caac.21166

2. Carney PA, Miglioretti DL, Yankaskas BC, et al. (2003) Individual and combined effects of age, breast density, and hormone replacement therapy use on the accuracy of screening mammography. Ann Intern Med 138:168-75.

3. Lobbes MBI, Smidt ML, Houwers J, et al. (2013) Contrast enhanced mammography: techniques, current results, and potential indications. Clin Radiol 68:935-44. doi: 10.1016/j.crad.2013.04.009

4. Heywang SH, Wolf A, Pruss E, et al. (1989) MR imaging of the breast with Gd-DTPA: use and limitations. Radiology 171:95-103. doi: 10.1148/radiology.171.1.2648479

5. Dromain C, Thibault F, Diekmann F, et al. (2012) Dual-energy contrast enhanced digital mammography: initial clinical results of a multireader, multicase study. Breast Cancer Res 14:R94. doi: $10.1186 / \mathrm{bcr} 3210$

6. Singh J, Daftary A (2008) Iodinated contrast media and their adverse reactions. J Nucl Med Technol 36:69-74; quiz 76-7. doi: 10.2967/jnmt.107.047621

7. Lawrence V, Matthai W, Hartmaier S (1992) Comparative safety of high-osmolality and lowosmolality radiographic contrast agents. Report of a multidisciplinary working group. Invest Radiol 27:2-28.

8. Cochran ST, Bomyea K, Sayre JW (2001) Trends in adverse events after IV administration of contrast media. AJR Am J Roentgenol 176:1385-8. doi: 10.2214/ajr.176.6.1761385

9. Caro JJ, Trindade E, McGregor M (1991) The risks of death and of severe nonfatal reactions with high- vs low-osmolality contrast media: a meta-analysis. AJR Am J Roentgenol 156:825-32. doi: 10.2214/ajr.156.4.1825900

10. Stacul F, van der Molen AJ, Reimer P, et al. (2011) Contrast induced nephropathy: updated ESUR Contrast Media Safety Committee guidelines. Eur Radiol 21:2527-41. doi: 10.1007/s00330-0112225-0

11. Lee B-Y, Ok J-J, Abdelaziz Elsayed AA, et al. (2012) Preparative fasting for contrast enhanced CT: reconsideration. Radiology 263:444-50. doi: 10.1148/radiol.12111605

12. Lewin JM, Isaacs PK, Vance V, Larke FJ (2003) Dual-energy contrast enhanced digital subtraction mammography: feasibility. Radiology 229:261-8. doi: 10.1148/radiol.2291021276

13. Kuhl CK, Schrading S, Bieling HB, et al. (2007) MRI for diagnosis of pure ductal carcinoma in situ: a prospective observational study. Lancet 370:485-92. doi: 10.1016/S0140-6736(07)61232-X

14. Diekmann F, Diekmann S, Jeunehomme F, et al. (2005) Digital mammography using iodine-based contrast media: initial clinical experience with dynamic contrast medium enhancement. Invest Radiol 40:397-404.

15. Dromain C, Balleyguier C, Muller S, et al. (2006) Evaluation of tumor angiogenesis of breast carcinoma using contrast enhanced digital mammography. AJR Am J Roentgenol 187:W528-37. doi: 10.2214/AJR.05.1944

16. Diekmann F, Freyer M, Diekmann S, et al. (2011) Evaluation of contrast enhanced digital mammography. Eur J Radiol 78:112-21. doi: 10.1016/j.ejrad.2009.10.002

17. Lehman CD, Gatsonis C, Kuhl CK, et al. (2007) MRI evaluation of the contralateral breast in women with recently diagnosed breast cancer. N Engl J Med 356:1295-303. doi: 10.1056/NEJMoa065447

18. Dromain C, Balleyguier C, Adler G, et al. (2009) Contrast-enhanced digital mammography. Eur J Radiol 69:34-42. doi: 10.1016/j.ejrad.2008.07.035

19. Dromain C, Thibault F, Muller S, et al. (2011) Dual-energy contrast-enhanced digital mammography: initial clinical results. Eur Radiol 21:565-74. doi: 10.1007/s00330-010-1944-y

20. Jochelson MS, Dershaw DD, Sung JS, et al. (2013) Bilateral contrast-enhanced dual-energy digital mammography: feasibility and comparison with conventional digital mammography and MR imaging in women with known breast carcinoma. Radiology 266:743-51. doi: 10.1148/radiol.12121084

21. Fallenberg EM, Dromain C, Diekmann F, et al. (2013) Contrast-enhanced spectral mammography versus MRI: Initial results in the detection of breast cancer and assessment of tumour size. Eur Radiol. doi: 10.1007/s00330-013-3007-7

22. Mann RM, Kuhl CK, Kinkel K, Boetes C (2008) Breast MRI: guidelines from the European Society of Breast Imaging. Eur Radiol 18:1307-18. doi: 10.1007/s00330-008-0863-7 
23. Boetes C, Veltman J, van Die L, et al. (2004) The role of MRI in invasive lobular carcinoma. Breast Cancer Res Treat 86:31-7. doi: 10.1023/B:BREA.0000032921.10481.dc

24. Hylton NM, Blume JD, Bernreuter WK, et al. (2012) Locally advanced breast cancer: MR imaging for prediction of response to neoadjuvant chemotherapy--results from ACRIN 6657/I-SPY TRIAL. Radiology 263:663-72. doi: 10.1148/radiol.12110748

25. Lobbes MBI (2012) Treatment response evaluation by MRI in breast cancer patients receiving neoadjuvant chemotherapy: there is more than just pathologic complete response prediction. Breast Cancer Res Treat 136:313-4. doi: 10.1007/s10549-012-2180-8

26. Prevos R, Smidt ML, Tjan-Heijnen VCG, et al. (2012) Pre-treatment differences and early response monitoring of neoadjuvant chemotherapy in breast cancer patients using magnetic resonance imaging: a systematic review. Eur Radiol 22:2607-16. doi: 10.1007/s00330-012-2653-5

27. (2010) Dutch national guidelines on family related breast and ovarian cancer. Consensus guidelines 2010 version 2.0. 1-7.

28. (2010) Dutch national guidelines on hereditary breast and ovarian cancer. Consensus guidelines 2010 version 2.0. 1-7.

29. Bluemke D, Gatsonis C (2004) Magnetic resonance imaging of the breast prior to biopsy. JAMA J ... 292:2735-2742.

30. Houssami N, Skaane P (2013) Overview of the evidence on digital breast tomosynthesis in breast cancer detection. Breast 22:101-8. doi: 10.1016/j.breast.2013.01.017

31. Froeling V, Diekmann F, Renz DM, et al. (2013) Correlation of contrast agent kinetics between iodinated contrast-enhanced spectral tomosynthesis and gadolinium-enhanced MRI of breast lesions. Eur Radiol 23:1528-36. doi: 10.1007/s00330-012-2742-5 



\section{Radiation exposure of contrast- enhanced spectral mammography compared to full field digital mammography}

Ulrich C. Lalji*

Cécile R. L. P. N. Jeukens*

Eduard Meijer

Betina Bakija

Robin Theunissen

Joachim E. Wildberger

Marc B. I. Lobbes

*Both authors contributed equally

Published in: Investigative Radiology 2014 


\section{Abstract}

Objectives: Contrast-enhanced spectral mammography (CESM) shows promising initial results, but comes at the cost of increased dose as compared to full-field digital mammography (FFDM). We aimed to quantitatively assess the dose increase of CESM in comparison to FFDM.

Materials and methods: Radiation exposure related data (such as kilovoltage, compressed breast thickness, glandularity, Entrance Skin Air Kerma (ESAK) and Average Glandular Dose (AGD) were retrieved for 47 CESM and 715 FFDM patients. All examinations were performed on one mammography unit. Radiation dose values reported by the unit were validated by phantom measurements. Descriptive statistics of the patient data were generated using a statistical software package.

Results: Dose values reported by the mammography unit were in good qualitative agreement with phantom measurements. Mean ESAK was $10.5 \mathrm{mGy}$ for a CESM exposure and $7.46 \mathrm{mGy}$ for a FFDM exposure. Mean AGD for a CESM exposure was $2.80 \mathrm{mGy}$ and $1.55 \mathrm{mGy}$ for a FFDM exposure.

Conclusions: Compared to our institutional FFDM, the AGD of a single CESM exposure is increased by $1.25 \mathrm{mGy}(+81 \%)$, whereas ESAK is increased by $3.07 \mathrm{mGy}$ $(+41 \%)$. Dose values of both techniques meet the recommendations for maximum dose in mammography. 


\section{Introduction}

Breast cancer is one of the most important causes of cancer-related deaths in women [1]. For example, it is estimated that more than 232,000 women in the United States will be diagnosed with breast cancer in 2013. An estimated 40,000 women will die of the disease in this year [2].

In breast cancer diagnostics, mammography is the most frequently used imaging modality [3, 4]. Diagnostic performance of mammography is good, but depends heavily on breast density [5]. Since the introduction of full-field digital mammography (FFDM), new techniques were developed to further improve mammography's diagnostic performance, such as contrast-enhanced spectral mammography (CESM).

A recent review on CESM summarized the promising initial results of this technique, which proved to be superior to conventional mammography [6]. In addition, recent studies have shown that CESM's accuracy is comparable to breast magnetic resonance imaging (MRI), which is currently considered to be the most accurate imaging modality for breast cancer staging $[7,8]$. Consequently, CESM might overcome limitations of conventional mammography, and might be considered as an alternative for breast MRI in the future. However, CESM uses ionizing radiation. Thus, in the discussion whether CESM can replace FFDM or breast MRI, radiation dose has to be considered. Our study objective was to assess radiation exposure of CESM compared to FFDM in the clinical setting.

\section{Materials and methods}

Between April and June 2013, 47 CESM and 715 FFDM exams were included consecutively. All examinations were performed on a single mammography system (Senographe* Essential with Senobright* (CESM) upgrade, GE Healthcare, Chalfont, United Kingdom). Radiation exposure related data such as tube voltage (kV), anode/filter combination, number of exposures, compressed breast thickness (mm), glandularity (\%), Entrance Skin Air Kerma (ESAK, in mGy) and Average Glandular Dose (AGD, in mGy), as set or determined by the mammography unit, were retrieved from the DICOM header of the images stored in our PACS system (IMPAX version 6.5, AGFA Healthcare, Mortsel, Belgium). The requirement for obtaining informed consent was waived by the local ethics committee.

For a CESM exam, patients are administered a non-ionic monomeric, lowosmolar contrast agent intravenously (iopromide; Ultravist $\AA$ 300, Bayer Healthcare, Berlin, Germany) two minutes prior to image acquisition, with a dose of $1.5 \mathrm{~mL} / \mathrm{kg}$ body weight, a flow rate $3 \mathrm{~mL} / \mathrm{s}$ and followed by a saline flush. The subsequent CESM exam consists of a low energy $(\mathrm{kVp}=26-31 \mathrm{kV}$ and target/filter combination $\mathrm{Mo} / \mathrm{Rh}$ or $\mathrm{Rh} / \mathrm{Rh}$ ), immediately followed by a high energy exposure $(\mathrm{kVp}=45-49 \mathrm{kV}$ and target/filter combination $\mathrm{Mo} / \mathrm{Cu}$ or $\mathrm{Rh} / \mathrm{Cu})$. In general, both 
breasts were examined in two views: mediolateral oblique (MLO) and craniocaudal (CC) views. Additional (special) views could be requested by the radiologist.

The low energy images are post-processed to obtain an image similar to a conventional mammogram. Recombined images are obtained after post-processing both low and high energy exposures and show enhancement information only. In the end, the radiologist is able to view both a 'conventional' mammogram (i.e. the low energy images) and contrast uptake (Figure 1).
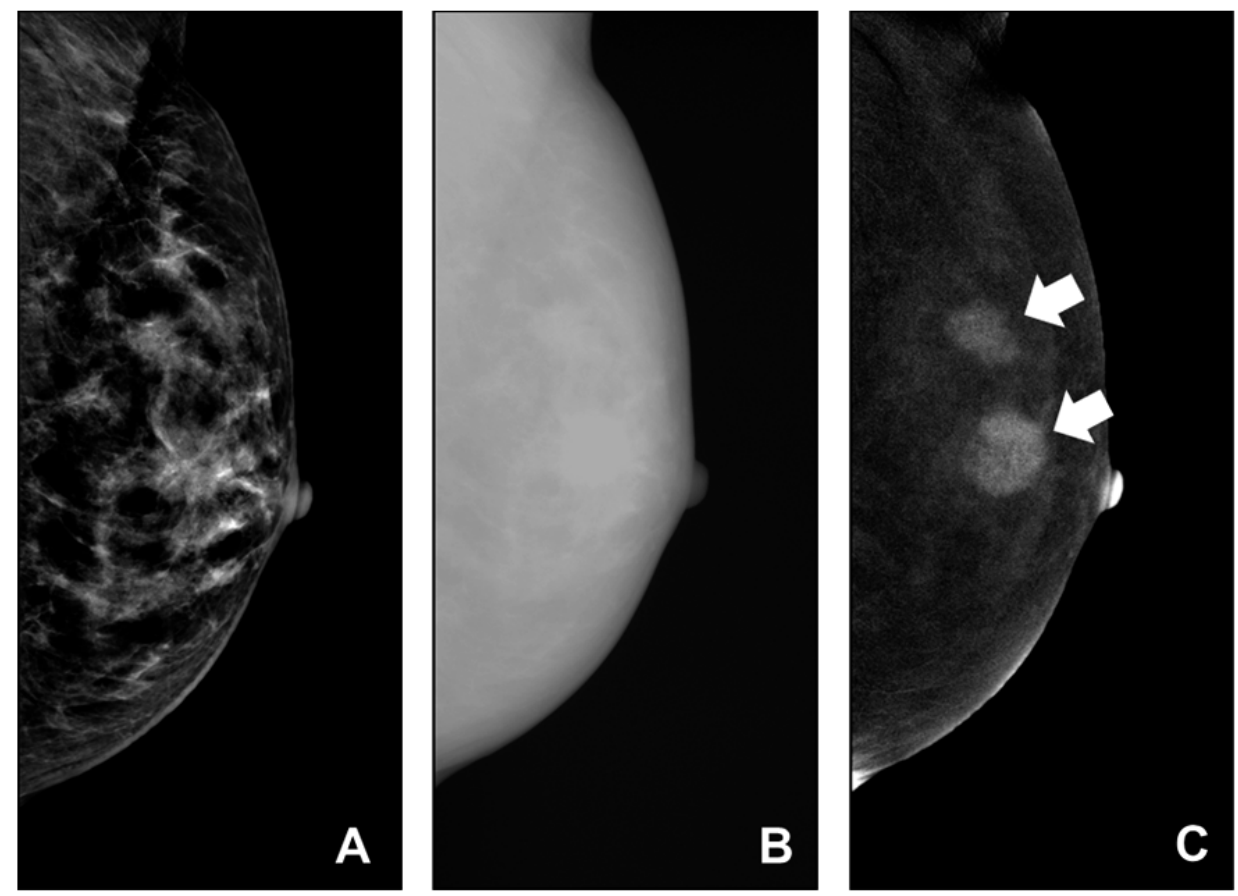

Figure 1: Typical example of a CESM exam, consisting of a (A) low-energy image (similar to a normal mammogram), a (B) high-energy image (non-diagnostic), and a (C) recombined image, showing areas of enhancement (arrows). Histopathology confirmed multifocal invasive breast cancer

The ESAK and AGD values recorded by the mammography unit were validated by phantom measurements. We used polymethyl methacrylate (PMMA) slab shaped phantoms with thicknesses of 30 to $70 \mathrm{~mm}$, which correspond to compressed breast thicknesses of 32 to $90 \mathrm{~mm}$ [9].

First, we made an exposure of the phantom without the dosimeter using the automatic exposure control (AEC) and recorded the exposure parameters. As our mammography unit uses a pre-shot in FFDM to determine exposure parameters, this experiment yields the exposure parameters for the corresponding compressed breast thickness. 
Second, the compression paddle was set to the corresponding breast thickness as this is used by the mammography unit to determine ESAK and AGD. For FFDM, the exposure was repeated in manual mode using the recorded parameters. For CESM, an exposure was made using the AEC (in 'CEDM' mode) to obtain the exposure parameters for the corresponding compressed breast thickness.

Third, we measured the air kerma using both an ion chamber and a solid state detector for these exposure parameters. We used a $1.5 \mathrm{~mL}$ ionization chamber (PS033) dedicated for measuring low energy spectra in mammography and a Capintec 192A electrometer (Capintec Inc. Ramsey, NJ). This system measures X-ray exposure in Roentgen (R), which was converted to air kerma in Gy (1 Gy $=0.8764 * 10-2$ $\mathrm{R})$. For CESM, the low energy and high energy exposure were determined separately by two measurements: once measuring low and high energy cumulative, and once measuring the low energy separately using the low energy exposure parameter CESM settings in the FFDM mode. The solid state detector dosimeter (Unfors RaySafe $^{\mathrm{TM}}$, Billdall, Sweden) was equipped with a dedicated mammography probe, calibrated for various target/filter combinations. For the high energy CESM exposure we used the so-called 'R/F-probe', suitable for the $>40 \mathrm{kV}$ range. The CESM exposure was performed twice, using both probes, while the accompanying software (Xi View version 2.0, Unfors RaySafe ${ }^{\mathrm{TM}}$, Billdall, Sweden) allowed separate recording of the low and high energy exposure dose parameters. The ionization chamber or probe were placed at the same position free in air, i.e. $14 \mathrm{~cm}$ above the detector with no phantom present, but below and in contact with the compression paddle. The measured air kerma was converted to ESAK at the entrance plane of the compressed breast using the inverse square law. The experiments above were repeated for phantom thicknesses of 30 to $70 \mathrm{~mm}$, which resulted in a clinical relevant range of tube voltages, tube currents and target/filter combinations for FFDM and CESM (Table 1).

For low energy exposures, our mammography unit uses the model published by Wu et al. to calculate AGD from ESAK [10]. This model incorporates breast glandularity, target/filter combination, tube voltage and output spectrum used. The latter is quantified by the half value layer, which was estimated from literature [9].

For high energy exposures, AGD cannot be calculated using the same model, since no normalized AGD values in this energy range have been published yet [10]. Alternatively, our mammography unit uses the monoenergetic model published by Boone et al. [11, 12], which is a more generic model that can assess AGD for any target/filter combination and tube voltages up to $50 \mathrm{kV}$. This model incorporates the same aforementioned parameters and has shown to agree with the $\mathrm{Wu}$ model [12]. Technical details of the implementation for the system's output spectrum were provided beforehand (GE Healthcare, internal publication). We validated AGD values reported by the mammography unit using measured ESAK values, the 
Wu or Boone model, and exposure parameters and glandularity provided by the mammography unit.

Descriptive statistics of the patient data were generated using SPSS Statistics (version 20.0, IBM, Armonk, NY). Differences between patient groups were evaluated using the independent samples t-test. P-values $<0.05$ were considered to be statistically significant. The phantom measurements are analyzed using the Excel software package (Microsoft, Redmond, WA).

\section{Results}

Patient characteristics and number of exposures are presented in Table 2. Patients examined with FFDM received a median of 4 exposures (range 1-10) and $92.6 \%$ of the exposures were standard CC and MLO projections. Patients examined with CESM also received a median of 4 exposures (range 4-6) of which 99\% were standard CC and MLO projections. In the final analysis only standard CC and MLO projections were considered. Mean exposure times of a single view CESM exposure for the low and high energy exposures were $1.4 \mathrm{~s}$ and $4.2 \mathrm{~s}$, respectively. For FFDM, mean exposure time was $1.1 \mathrm{~s}$.

Figure 2 shows the AGD as a function of equivalent compressed breast thickness for low- and high-energy CESM exposures, as well as FFDM (including their exponential fits). The AGD increases for increased compressed breast thickness for both FFDM and CESM exams. Table 3 shows the ESAK and AGD per FFDM and CESM exposure (low energy, high energy and combined). For FFDM the AEC mode was 'standard' in $85 \%$ of the exams and 'contrast' in $15 \%$ of the exams. Mean ESAK and AGD for a single CESM exposure were $10.5 \mathrm{mGy}$ and $2.80 \mathrm{mGy}$, respectively. Compared to a conventional FFDM exposure, ESAK and AGD of a complete CESM exposure were $41 \%$ and $81 \%$ higher, respectively. The high energy exposure contributed $7 \%$ and $24 \%$ to the total ESAK and AGD of a CESM exam, respectively.

Table 1: Settings of exposure parameters in the phantom experiments. CESM indicates contrastenhanced spectral mammography; FFDM, full-field digital mammography; PMMA, polymethyl methacrylate.

\begin{tabular}{|c|c|c|c|c|c|c|c|c|c|c|}
\hline \multirow[b]{2}{*}{$\begin{array}{l}\text { PMMA } \\
\text { phantom } \\
\text { thickness } \\
(\mathrm{mm})\end{array}$} & \multirow[b]{2}{*}{$\begin{array}{l}\text { Equivalent } \\
\text { compressed } \\
\text { breast } \\
\text { thickness } \\
(\mathrm{mm})\end{array}$} & \multicolumn{3}{|c|}{ FFDM } & \multicolumn{3}{|c|}{ CESM low energy } & \multicolumn{3}{|c|}{ CESM high energy } \\
\hline & & $\mathrm{kV}$ & $\mathrm{mAs}$ & $\begin{array}{l}\text { Target/ } \\
\text { Filter } \\
\text { material }\end{array}$ & $\mathrm{kV}$ & $\mathrm{mAs}$ & $\begin{array}{l}\text { Target/ } \\
\text { Filter } \\
\text { material }\end{array}$ & $\mathrm{kV}$ & mAs & $\begin{array}{l}\text { Target/ } \\
\text { Filter } \\
\text { materia }\end{array}$ \\
\hline 30 & 32 & 26 & 50 & Mo/Mo & 28 & 71 & Mo/Rh & 45 & 125 & $\mathrm{Mo} / \mathrm{Cu}$ \\
\hline 40 & 45 & 27 & 63 & Mo/Rh & 29 & 71 & $\mathrm{Rh} / \mathrm{Rh}$ & 45 & 180 & $\mathrm{Rh} / \mathrm{Cu}$ \\
\hline 50 & 60 & 29 & 63 & $\mathrm{Rh} / \mathrm{Rh}$ & 31 & 80 & $\mathrm{Rh} / \mathrm{Rh}$ & 47 & 200 & $\mathrm{Rh} / \mathrm{Cu}$ \\
\hline 60 & 75 & 29 & 110 & $\mathrm{Rh} / \mathrm{Rh}$ & 30 & 140 & $\mathrm{Rh} / \mathrm{Rh}$ & 49 & 200 & $\mathrm{Rh} / \mathrm{Cu}$ \\
\hline 70 & 90 & 31 & 125 & $\mathrm{Rh} / \mathrm{Rh}$ & 30 & 140 & $\mathrm{Rh} / \mathrm{Rh}$ & 49 & 200 & $\mathrm{Rh} / \mathrm{Cu}$ \\
\hline
\end{tabular}


Table 2: Patient Characteristics and Number of Exposures. No.CC indicates craniocaudal; CESM, contrast-enhanced spectral mammography; FFDM, full-field digital mammography; MLO, mediolateral oblique; Max, maximum; Min, minimum.

\begin{tabular}{|c|c|c|c|c|c|c|c|c|}
\hline & \multicolumn{4}{|l|}{ FFDM } & \multicolumn{4}{|l|}{ CESM } \\
\hline & Mean & SD & Min & Max & Mean & SD & Min & $\operatorname{Max}$ \\
\hline Number of patients & \multicolumn{4}{|l|}{715} & \multicolumn{4}{|l|}{47} \\
\hline Total number of exposures & \multicolumn{4}{|l|}{2782} & \multicolumn{4}{|l|}{195} \\
\hline Age (years) & 56 & 13 & 23 & 93 & 58 & 8 & 50 & 74 \\
\hline Breast thickness (mm) & 56.1 & 13.6 & 15.0 & 100 & 58.4 & 13.7 & 21.0 & 96 \\
\hline Glandularity (\%) & 42 & 27 & 0 & 100 & 37 & 28 & 0 & 100 \\
\hline Projection & \multicolumn{4}{|c|}{ CC I MLO (1238 / 1339) } & \multicolumn{4}{|c|}{ CC I MLO (96 / 97) } \\
\hline
\end{tabular}

In the CESM-group, mean breast thickness was $2.3 \mathrm{~mm}$ higher than in the FFDMgroup ( $\mathrm{p}=0.025)$, whereas glandularity was $5 \%$ lower $(\mathrm{p}=0.015)$. Using the regression curve, we calculated that a $2.3 \mathrm{~mm}$ increase of breast thickness would lead to a 4\% and 5-6\% increase in AGD for FFDM and CESM, respectively.

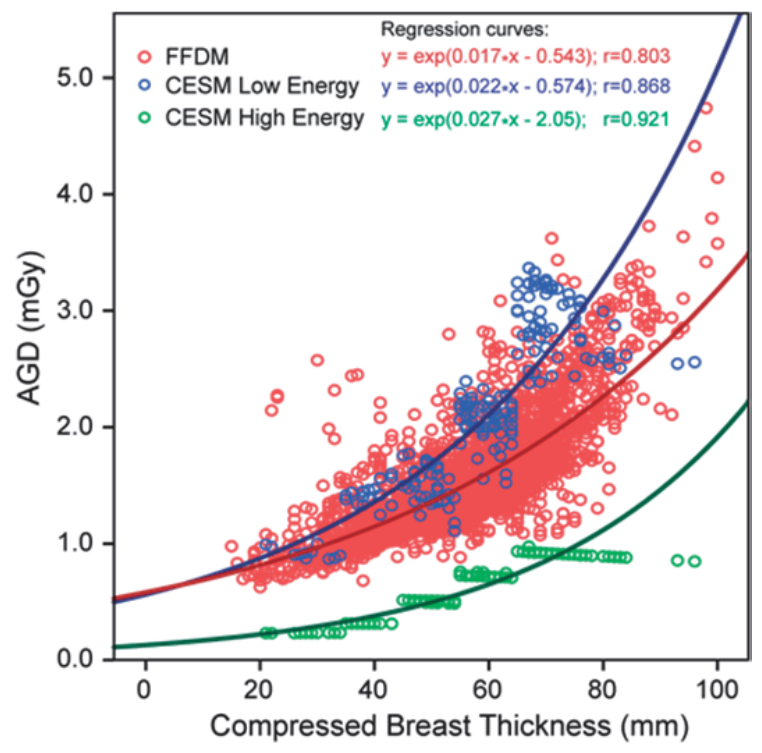

Figure 2: Dependence of AGD on the compressed breast thickness for the FFDM and low-energy and high-energy CESM.

In other words, for FFDM an increase from 56.1 to $58.4 \mathrm{~mm}$ breast thickness corresponds to a $0.06 \mathrm{mGy}$ increase of AGD. Considering the uncertainties involved in calculating AGD, it is our opinion that although the differences in these parameters were statistically significant (largely resulting from the higher number of exposures in the FFDM-group), they were not significant clinically. 
Table 3: ESAK and AGD per FFDM and CESM exposure. Number of exposures includes both CC and MLO. AGD indicates average glandular dose; CESM, contrast-enhanced spectral mammography; ESAK, entrance skin air kerma; FFDM, full-field digital mammography; Max, maximum; Min, minimum.

\begin{tabular}{|c|c|c|c|c|c|}
\hline & & Mean & SD & Min & Max \\
\hline \multicolumn{6}{|l|}{ FFDM } \\
\hline Number of exposures & 2577 & & & & \\
\hline ESAK & (mGy) & 7.46 & 3.43 & 2.03 & 33.0 \\
\hline AGD & (mGy) & 1.55 & 0.48 & 0.63 & 5.12 \\
\hline \multicolumn{6}{|l|}{ CESM Low Energy } \\
\hline Number of exposures & 193 & & & & \\
\hline ESAK & (mGy) & 9.80 & 3.89 & 3.13 & 15.97 \\
\hline AGD & (mGy) & 2.12 & 0.68 & 0.87 & 3.37 \\
\hline \multicolumn{6}{|l|}{ CESM High Energy } \\
\hline Number of exposures & 193 & & & & \\
\hline ESAK & (mGy) & 0.73 & 0.27 & 0.21 & 1.12 \\
\hline AGD & (mGy) & 0.68 & 0.22 & 0.23 & 0.98 \\
\hline \multicolumn{6}{|l|}{ CESM Low + High Energy } \\
\hline Number of exposures & 193 & & & & \\
\hline ESAK & (mGy) & 10.5 & 4.15 & 3.34 & 17.1 \\
\hline AGD & (mGy) & 2.80 & 0.88 & 1.10 & 4.29 \\
\hline
\end{tabular}

Figure 3 shows the results of ESAK measurements compared to ESAK values reported by the unit. As expected, ESAK increases with increasing compressed breast thickness. Table 4 shows the range of relative and absolute differences for ESAK and AGD values determined from the phantom measurements compared to the values reported by the mammography unit. The range of AGD values in the phantom experiments was 1.21-2.48 mGy for FFDM, 1.89-2.96 mGy for low energy CESM, and 0.15-1.04 mGy for high energy CESM. In short, the measured values obtained by the ion chamber and solid state detector are consistent and show good qualitative agreement with the mammography unit values. The observed variation, however, should be taken into account when interpreting our results.

\section{Discussion}

In this study, we have assessed mean ESAK and AGD of a single CESM exposure in comparison to a single FFDM exposure. For CESM, we found a mean ESAK and mean AGD of 10.5 mGy and 2.80 mGy which is an increase of 3.07 mGy (41\%) and 1.25 mGy (81\%), respectively, compared to our institutional FFDM. As with any radiation exposure, medical benefits must be outweighed to the additional risk of health detriment due to this increase in dose.

CESM became commercially available in 2011. Initial studies have shown that contrast-enhanced spectral mammography is superior to FFDM [6], because additional information on enhancement of structures is available for the radiologist. 
This principle of (tumour) enhancement has been long used in breast MRI evaluation [13], and seems to contribute in CESM as well.

Table 4. Comparison of the ESAK measurements and calculated AGD based on ESAK measurements with the ESAK and AGD values reported by the mammography unit. The comparison is given as a range of relative and absolute differences for the ion chamber and solid state detector measurements

\begin{tabular}{|c|c|c|c|c|}
\hline & Ion chamber (\%) & Solid State (\%) & Ion chamber (mGy) & Solid State (mGy) \\
\hline \multicolumn{5}{|l|}{$E S A K$} \\
\hline FDM/Low energy CESM & $21-25 \%$ & $12-14 \%$ & $0.9-3.3$ & $0.7-2.08$ \\
\hline High energy CESM & $29-67 \%$ & $-35-10 \%$ & $0.1-0.3$ & $-0.07-+0.07$ \\
\hline \multicolumn{5}{|l|}{$A G D$} \\
\hline FFDM/Low energy CESM & $14-37 \%$ & $5-23 \%$ & $0.29-0.58$ & $0.12-0.50$ \\
\hline High energy CESM & $28-67 \%$ & $-35-10 \%$ & $0.15-0.28$ & $-0.08-+0.06$ \\
\hline
\end{tabular}

Two studies have compared CESM and breast MRI $[7,8]$. In 52 cases, Jochelson et al. showed that breast cancer detection rate was similar for CESM and MRI: $96 \%$ [7]. In addition, they showed that CESM suffered from less false-positive findings than MRI, but MRI was more accurate in the assessment of tumour multifocality. Fallenberg et al. showed a breast cancer detection rate of $99.7 \%$ for MRI, whereas CESM detected all cancers [8]. In both studies the diagnostic accuracy of CESM was superior to conventional mammography, as already reported earlier [6]. Fallenberg et al. also studied the correlation between tumour diameter measurements of CESM versus MRI, with histopathological measurements as the gold standard. For CESM versus histopathology, Pearson's correlation coefficient was 0.733 , whereas for MRI versus histopathology it was 0.654 . These studies show that CESM has the potential to replace conventional mammography or breast MRI in the future.

Only a few studies have reported AGD measurements of CESM in relation to FFDM examinations. Fallenberg et al. reported a lower dose for CESM than mammography: 1.72 mGy versus 1.75 mGy, respectively [8]. The explanation provided was that conventional mammography exams consisted of exams by multiple vendors and both CR and DR images (Fallenberg, personal communication). On the other hand, Dromain et al. reported an increase in radiation dose of $20 \%$ compared to FFDM [13]. Although the dose range reported for a complete CESM exam (0.7$3.6 \mathrm{mGy}$ ) was comparable to our findings (1.1-4.4 mGy), we observed a dose increase of $81 \%$ compared to our institutional FFDM. Objective comparisons between these results and our current observations are limited as both Dromain et al. and Fallenberg et al. used a prototype CESM unit in which exposure settings were manually set depending on breast thickness and glandularity using a Table of predefined exposure values $[8,13])$, whereas we used the commercially available product in which CESM settings are determined by the AEC.

Badr et al. found similar dose measurements for CESM (mean 2.65 mGy per image) and concluded that it was increased with 54\% compared to FFDM (1.72 mGy) [14]. Since our FFDM dose is $1.55 \mathrm{mGy}$, our relative increase compared to FFDM was 
81\%. CESM dose is still within acceptable ranges for mammography. However, if one would compare a CESM exposure to other techniques, such as photon-counting full-field digital mammography [15] (0.74 mGy) or contrast-enhanced low-dose photon-counting tomosynthesis [16] (0.42 mGy), the dose increase would even be $278 \%$ and $567 \%$, respectively. These different percentages might cause unnecessary confusion and show that it is important to report both CESM and FFDM dose values (in $\mathrm{mGy}$, not in percentages of a certain reference standard).

The health detriment due to radiation exposure can be estimated from the AGD and age dependent Lifetime Attributable Risk (LAR) factors for breast cancer incidence and mortality published by the Biological Effects of Ionizing Radiation (BEIR) VII committee [17]. Table 5 shows the LAR for a unilateral, single FFDM and CESM exposure, demonstrating a small risk compared to the lifetime risk of breast cancer incidence and mortality of 12,000 and 3,000 cases per 100,000 women, respectively [17]. However, it should be emphasized that these are the numbers of a unilateral, single exposure, not a complete exam.

We report both ESAK and AGD in our study. ESAK is a parameter that can be objectively measured using the proper setup. In contrast, AGD is to be calculated from the ESAK using a model such as the Wu or Boone model, which are based on Monte Carlo calculations of a modelled breast and mammography unit. Such calculations involve making assumptions about breast composition and thickness.

Nevertheless, the AGD is the accepted measure for dose absorbed in the breast [9], and (as opposed to ESAK) can be used for health detriment estimations using LAR values presented in Table 4. However, it should be noted that for the low dose regime, as is the case here, LAR values are extrapolated from epidemiological studies involving higher doses (>100mGy) $[17,18]$ using a linear, non-threshold model. Consequently, the estimated risks should be interpreted with caution, i.e. rather as an order of magnitude than an absolute value.

Table 5. Life Attributable Risk for breast cancer incidence and mortality for a single, unilateral FFDM $(\mathrm{AGD}=1.55 \mathrm{mGy})$ and CESM (AGD = $2.80 \mathrm{mGy}$ ) exposure. In case the complete examination consists of for example four exposures (CC and MLO for both breasts), the risks are to be multiplied by four.

\begin{tabular}{llll}
\hline & Age (years) & $\begin{array}{l}\text { Incidence } \\
\text { (number of cases/ 100,000 persons) }\end{array}$ & $\begin{array}{l}\text { Mortality } \\
\text { (number of cases/ 100,000 persons) }\end{array}$ \\
\hline FFDM & 40 & 1.1 & 0.3 \\
& 60 & 0.2 & $<0.1$ \\
& 80 & $<0.1$ & $<0.1$ \\
CESM & 40 & 2.0 & 0.5 \\
& 60 & 0.4 & 0.2 \\
& 80 & $<0.1$ & $<0.1$ \\
\hline
\end{tabular}


For our mammography unit the ESAK and AGD for the low energy CESM exposure are higher than the FFDM exposure for compressed breast thicknesses 30-75 mm. This is attributable to a different setting of the AEC, resulting in a different optimal setting of the target/filter material, $\mathrm{kV}$ and mAs settings for FFDM and low energy CESM (Table 1). These results suggest that further dose optimization, especially for the low-energy CESM exposures, should be possible. However, the low energy CESM exposure is part of a dual-energy CESM exposure. Lowering the ESAK might have consequences for the reconstructed contrast-enhanced images. In addition, the optimal CESM exposure settings also depend on the image quality needed for a specific diagnostic problem: for example, whether it is necessary to observe micro calcifications, or does the radiologist only require the lesion enhancement information. If not, a lower image quality might be acceptable in favour of a lower dose. With respect to dose optimization, multiple variables (contrast agent and target/filter material used, $\mathrm{kV}, \mathrm{mAs}$ ) can be studied. Technical improvements will most likely decrease CESM dose in the future, but we were unable to perform any further dose optimization experiments, since the unit does not allow the user to adjust the AEC modes due to safety precautions. Since the main study goal was to provide the reader with an independent dose overview of this commercially available product, we felt that further dose optimization studies were beyond the scope of this article.

We performed phantom measurements with both an ionization chamber and a solid state detector. The latter type of detectors usually shows significant energy dependence towards lower energies. Therefore, care must be taken that the detector is properly calibrated for this energy range. Our results show that when this is the case, solid state detector measurements are indeed consistent with both ionization chamber measurements and values reported by the mammography unit.

Our mammography unit uses the Wu model to calculate the AGD for low energy exposures. Alternatively, in the European Guidelines [9], the Dance model is recommended to determine AGD $[19,20]$, which is also based on Monte Carlo modelling, but which presents a different approach in how AGD can be calculated by a user. We calculated AGD also using this model and found good agreement (i.e. within $10 \%$ ) with AGD values obtained using the Wu model. However, the observed differences in AGD between the two methods are indicative of the uncertainties inherent to using any model, which should be realized when interpreting AGD values.

A limitation of this study is that we were not able to objectively compare dose received for an entire breast exam (standard and additional views combined). In our hospital, CESM is used as a case solving imaging modality only in women referred from the breast cancer screening program. This CESM population is different from the one receiving FFDM, of whom the majority consists of (annual) follow-up or screening. This prohibits a valid comparison. Furthermore, we started using CESM on January 1st, 2013. Consequently, the CESM learning curve might have influenced requests of additional CESM views in the study period. Nevertheless, the diagnostic accuracy of mammography is increased by CESM, and it might 
be expected that less additional special views (such as compression or lateral views) would be needed in CESM, decreasing the difference in total dose acquired for complete breast exams. Although this is somewhat reflected by the smaller range of total number of exposures in our FFDM patients (1-10, versus 4-6 in CESM patients), we felt that before mentioned limitations hampered an objective comparison of the dose acquired per complete exam. Therefore, we opted to present the dose acquired in a single exposure for FFDM and CESM, which has the additional benefit that comparison with previous studies is possible.

In conclusion, studies have shown that diagnostic accuracy of CESM is higher than FFDM and might even be comparable to breast MRI. Nonetheless, it comes at the price of an increased radiation dose. Dose values of both techniques meet the recommendations for maximum dose in mammography. Taking into account that the results provided so far are very promising for CESM, the benefits of this method by far outreach the extra risks compared to FFDM. Nevertheless, dose considerations should always be conducted on a personal level, especially in young women where breast MRI has proven to be a robust and reliable alternative in the clinical setting. 


\section{References}

1. Siegel R, Naishadham MA, Jemal A. Cancer statistics, 2013. CA Cancer J Clin. 2013;63:11-30.

2. Breast cancer. [National Cancer Institute web site]. Available at: http://www.cancer.gov/cancertopics/types/breast. Accessed December 15, 2013.

3. Yoon KH, Kwon YM, Choi BJ, et al. Monochromatic x-rays for low-dose digital mammography: preliminary results. Invest Radiol 2012;47:683-687.

4. Sardanelli F, Podo F, Santoro F, et al. Multicenter surveillance of women at high genetic breast cancer risk using mammography, ultrasonography, and contrast-enhanced magnetic resonance imaging (the high breast cancer risk Italian 1 study): final results. Invest Radiol 2011;46:94-105.

5. Carney PA, Miglioretti DL, Yankaskas BC, et al. Individual and combined effects of age, breast density, and hormone replacement therapy use on the accuracy of screening mammography. Ann Intern Med. 2003;138:168-175.

6. Lobbes MBI, Smidt ML, Houwers J, et al. Contrast-enhanced spectral mammography: techniques, current results and potential indications. Clin Radiol. 2013;68:935-944.

7. Jochelson MS, Dershaw DD, Sung JS, et al. Bilateral contrast-enhanced dual-energy digital mammography: feasibility and comparison with conventional digital mammography and MR imaging in women with known breast carcinoma. Radiology. 2013;266:743-751.

8. Fallenberg EM, Dromain C, Diekmann F, et al. Contrast-enhanced spectral mammography versus MRI: initial results in the detection of breast cancer and assessment of tumour size. Eur Radiol. 2013; Sep 19

9. European Communities. European guidelines for quality assurance in breast cancer screening and diagnosis, 4th edition. Luxembourg. Office for Official Publications of the European Communities; 2006.

10. Wu X, Gingold EL, Barnes GT, Tucker DM. Normalized average glandular dose in molybdenum targetrhodium filter and rhodium target-rhodium filter mammography. Radiology. 1994;193: 83-89.

11. Boone JM. Normalized glandular dose ( $\mathrm{DgN})$ coefficients for arbitrary $x$-ray spectra in mammography: computer-fit values of Monte Carlo derived data. Med Phys. 2002;29:869-875.

12. Boone JM. Glandular breast dose for monoenergetic and high-energy x-ray beams: Monte Carlo assessment. Radiology. 1999;213:23-37.

13. Kuhl C. The current status of breast MR imaging. Part I. Choice of technique, image interpretation, diagnostic accuracy, and transfer to clinical practice. Radiology. 2007;244:356-378.

14. Badr S, Laurent N, Régis C, et al. Dual-energy contrast-enhanced digital mammography in routine clinical practice in 2013. Diagn Interv Imaging. 2013;Nov 12, Epub ahead of print.

15. Cole EB, Toledano AY, Lundqvist M, Pisano ED. Comparison of radiologist performance with photon-counting full-field digital mammography to conventional full-field digital mammography. Acad Radiol 2012;19:916-922.

16. Schmitzberger FF, Fallenberg EM, Lawaczeck R, et al. Development of low-dose photon-counting contrast-enhanced tomosynthesis with spectral imaging. Radiology 2011;259:558-564.

17. National Research Council. Health risks from exposure to low levels of ionizing radiation: BEIR VII phase 2. Washington, DC: National Academic Press; 2006.

18. Valentin J. The 2007 recommendations of the International Commission on Radiological Protection. Ann ICRP 2007;37:1-332.

19. Dance DR. Monte Carlo calculation of conversion factors for the estimation of mean glandular breast dose. Med Phys Biol 1990;35:1211-1219.

20. Dance DR, Skinner CL, Young KC, et al. Additional factors for the estimation of mean glandular breast dose using the UK mammography dosimetry protocol. Phys Med Biol 2000;45:3225-3240. 



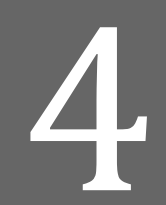

\section{Evaluation of low-energy contrast- enhanced spectral mammography images by comparing them to full-field digital mammography using EUREF image quality criteria}

Ulrich C. Lalji

Cécile R. L. P. N. Jeukens

Ivo P. L. Houben

Patty J. Nelemans

Ruben E. van Engen

Emmely van Wylick

Regina G. H. Beets-Tan

Joachim E. Wildberger

Leonie E. Paulis

Marc B. I. Lobbes

Published in : European Radiology 2015 


\section{4 | Chapter 4}

\section{Abstract}

Objective: A CESM exam results in a low-energy image (LE) and a contrastenhanced image. The LE appears similar to a full-field digital mammogram (FFDM). Our aim is to evaluate LE CESM image quality by comparing it to FFDM using criteria defined by the European Reference Organization for Quality Assured Breast Screening and Diagnostic Services (EUREF).

Methods: A total of 147 cases with both FFDM and LE images were independently scored by two experienced radiologists using these (20) EUREF criteria. Contrast detail measurements were performed using a dedicated phantom. Differences in image quality scores, average glandular dose, and contrast detail measurements between LE and FFDM were tested for statistical significance.

Results: No significant differences in image quality scores were observed between LE and FFDM images for 17 out of 20 criteria. LE scored significantly lower on one criterion regarding the sharpness of the pectoral muscle $(\mathrm{p}<0.001)$. LE scored significantly better on two criteria on the visualization of micro calcifications $(\mathrm{p}=0.02$ and $p=0.034$ ). Dose and contrast detail measurements did not reveal any physical explanation for these observed differences.

Conclusions: Low-energy CESM images are non-inferior to FFDM images. From this perspective FFDM can be omitted in patients with an indication for CESM. 


\section{Introduction}

In breast imaging, mammography continues to play an important role. The diagnostic performance of mammography is good, but depends heavily on breast density [1]. With the introduction of digital mammography, several applications that increase diagnostic accuracy (even in dense breasts) have been developed. One of these is contrast-enhanced spectral mammography (CESM). Recent studies continue to show that CESM is superior to conventional mammography in breast cancer detection [2-5].

A standard CESM exam consists of three sets of images per exposure: a low energy image (LE); a high energy image; and a recombined image (Figure 1). The LE image is acquired a peak kilovoltage $(\mathrm{kVp})$ values ranging from $26-31 \mathrm{kVp}$. In this way, the entire $\mathrm{x}$-ray spectrum is below the k-edge of iodine (which is 33.2 $\mathrm{keV}$ ) [3]. As a result, the LE image resembles a full-field digital mammogram (FFDM), although iodine contrast is already present within the breast. However, in order to omit FFDM in cases for which CESM is indicated (for example in postmenopausal women with a highly suspicious palpable breast mass), LE images have to comply with the high image quality standards used in breast imaging and should at least be non-inferior to their FFDM counterpart. The high energy image is not used for diagnostic purposes, but is used in post-processing to create the recombined image, in which areas of enhancement can be appreciated.

In this retrospective study, we aimed to study the differences between LE and FFDM images in a standardized and structured manner. Our primary study aim was to evaluate the image quality of LE images using FFDM acquired at Dutch Breast Cancer Screening Program (DBCSP) as a reference standard and using the image quality criteria defined by the European Reference Organization for Quality Assured Breast Screening and Diagnostic Services (EUREF) [6]. Our secondary study aim was to evaluate the differences in dose and contrast detail measurements between both images. 


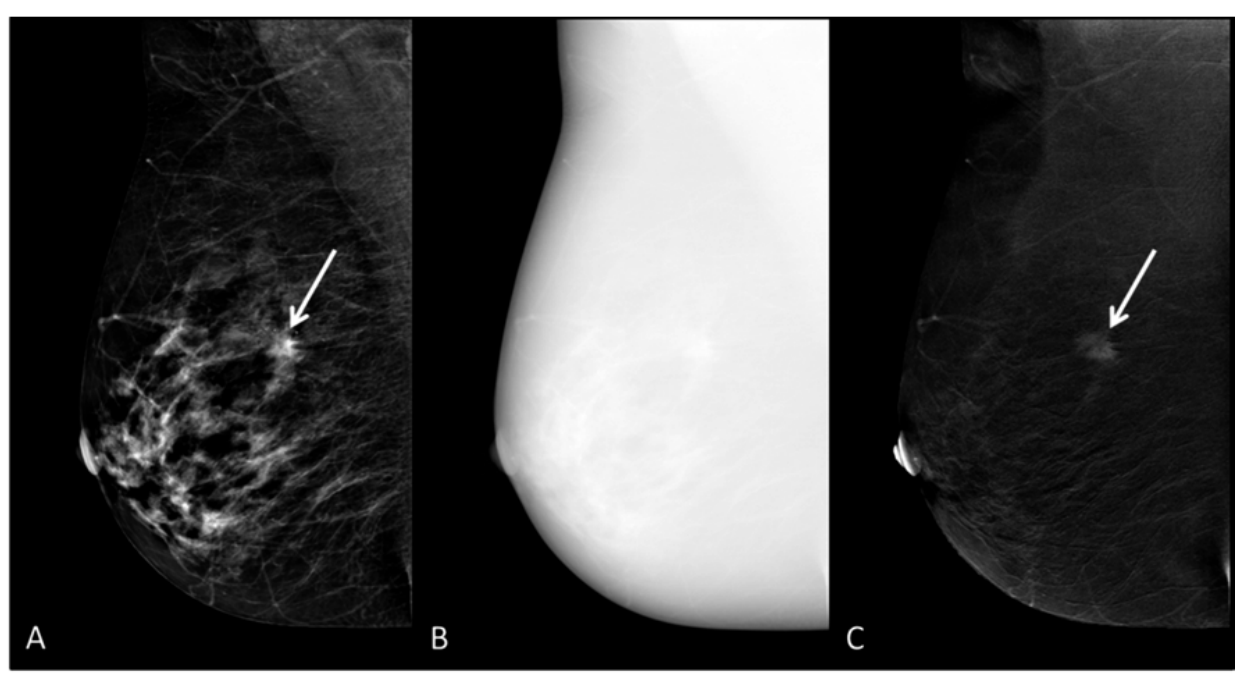

Figure 1. A typical CESM examination (only right mediolateral oblique view shown), consisting of a low-energy (A), high-energy (B), and recombined image (C). A suspicious lesion is seen on the lowenergy image, showing enhancement on the recombined image (white arrows). Histopathology showed invasive ductal carcinoma. The high-energy image is not for diagnostic purpose but used for construction of the recombined image.

\section{Materials and methods}

In the Netherlands, women (aged 50-75 years) are invited to participate in the DBCSP every two years [7]. If a breast abnormality is detected, women are referred to a hospital of their choice for further analysis. In our institute, every recalled patient is eligible to undergo CESM, unless contra-indications for the use of iodinebased contrast agents exist, such as an increased risk of developing contrastinduced nephropathy or known contrast allergies.

In this study, data from women that underwent CESM for a screen-detected breast abnormality were collected in the period of July 2013 till February 2014. Since they were recalled from screening, all women had a recent (screening) FFDM exam for comparison. The need for informed consent was waived by the local ethical committee.

\section{Image acquisition and analysis}

FFDM was performed on a standard mammography unit used in the DBCSP (Hologic Selenia, Bedford, MA, USA), whereas LE images were acquired as part of a CESM (Senographe* Essential with Senobright* upgrade, GE Healthcare, Chalfont St Giles, United Kingdom). All images were displayed on a dedicated mammography workstation (IDI MammoWorkstation 4.7.0, GE Healthcare, Chalfont St Giles, United Kingdom) equipped with mammography approved monitors (Barco Co- 
ronis 5MP Mammo, Barco, Kortrijk, Belgium). Two experienced radiologists (U.L. and M.L., both viewing over 6.000 clinical and screening mammographies annually) independently scored the images using 20 image quality criteria defined by EUREF (Table 1).

These are criteria concerning overall diagnostic value, visibility of (micro) calcifications, tissue visibility at chest wall and at the skin line, overall density as well as sharpness / noise impression [6]. The radiologists were not allowed to change window width or level settings while scoring the images.

The original EUREF criteria are dichotomous and can only be answered with 'yes' or 'no'. In order to perform a more detailed analysis, we decided to use a 5point scoring scale, with ' 1 ' being the least favourable score and ' 5 ' the best possible score for each item. For example, on the question 'Are the vascular structures visible through the dense parenchyma?' a score of ' 1 ' would mean that vascular structures were hardly visible while a score of ' 5 ' would indicate that visibility was excellent.

For scoring, the images were divided in two datasets, each containing either the FFDM or LE of a patient. Patients were randomized with respect to the order in which images were evaluated: LE first and FFDM second, or vice versa. Randomization was performed to prevent recall bias. The LE and FFDM images were viewed separately by both radiologists with a six-week interval between the scoring of the first and second image set. Both radiologists were blinded for each other's scores. In case of discrepancies consensus was reached by both radiologists favouring the lowest score given for that criterion.

\section{Dose measurements}

The Entrance Surface Air Kerma (ESAK) and Average Glandular Dose (AGD) were determined according to the method described in European guidelines [4]. In short, tube output of both mammography units was determined by measuring the air kerma using a dosimeter with a dedicated mammography probe calibrated for the target/filter combinations used in our measurements (Unfors RaySafe; Billdall, Sweden). The probe was placed on the bucky table below the compression paddle touching it.

Tube output was determined with current-time product 50 mAs (CESM) / 70 mAs (FFDM) for all target/filter combinations observed in respectively the LE and FFDM patient group. For each target/filter combination tube output measurements of three tube voltages covering the whole clinically used range were measured. For the same spectra the half-value layer (HVL) was also measured using the dosimeter in the setup described in the European Guidelines. The technical parameters required for the ESAK and AGD calculation, i.e. $\mathrm{kV}$, target, filter, current-time product, and compressed breast thickness (CBT) were obtained for each exposure from the DICOM header of the images. The ESAK was calculated using the appropriate 
time-current product and tube output, which was corrected for breast thickness using the inverse square law. Next, the AGD was calculated from the ESAK using the Dance model $[6,9,10]$.

\section{Contrast detail phantom measurements}

Contrast detail measurements were performed using a CDMAM phantom (version 3.4, Artinis Medical Systems, Elst, The Netherlands), which consists of a matrix of gold disks of diameter range 0.06 to $2.0 \mathrm{~mm}$ and thickness range 0.03 to $2.0 \mu \mathrm{m}$. The phantom was positioned in the middle of a stack of polymethylmethacrylate (PMMA) plates of varying thicknesses to simulate the clinical range of CBT as described in the EUREF guidelines [6], where the CDMAM phantom corresponds to $10 \mathrm{~mm}$ PMMA thickness. To determine the exposure parameters for each thickness, i.e. target/filter combination, tube voltage, and current-time product, a FFDM or LE exposure was made in fully automatic exposure control mode. Using these exposure parameters 16 exposures were made for each simulated breast thickness. The unprocessed images were exported to the analysis software (CDMAM analyzer, version 2.0.5, Elst, The Netherlands), which analyses the threshold detectability of the gold disks in each image, resulting in an average threshold thickness for each disc diameter. Subsequently, we plotted the threshold detection thickness versus the disc diameter on a log-log scale and generated a contrastdetail curve by performing a fit.

\section{Statistical analyses}

For each of the 20 EUREF criteria, the frequency of the (optimal) '5' score was calculated. Any statistically significance between the scores of LE and FFDM images were assessed by using a McNemar test for paired proportions. In addition, median scores of LE and FFDM images were calculated, using a Wilcoxon signed rank test to test for statistically significant differences. In case of micro calcifications, if none were observed, ratings (criteria 5 and 18) were scored as 'not applicable'.

For the dose measurements, AGD values of the entire patient population were tested pairwise for differences between FFDM and LE exposures using a Wilcoxon matched-pair signed rank test. Furthermore, we studied whether differences in AGD depended on breast thickness. To this extent, mean CBT was calculated for each patient by averaging the CBT of all exposures taken on both systems, which were four FFDM and four LE exposures (CC and MLO orientation of left and right breast). Patients were categorized according to their mean CBT and the AGD was statistically tested for differences between the FFDM and LE group using a paired sample t-test.

To compare the detection curves between FFDM and LE in the contrast detail measurements a multivariable linear regression analysis was performed to evaluate detection threshold thickness differences for LE and FFDM. The mammography 
method (FFDM or LE) and 1/diameter of the gold discs were used as covariates, the latter as a first order approximation of the theoretical relation stated in the European guidelines [4].

All p-values $<0.05$ were considered to be statistically significant. All statistical analyses were performed using SPSS (IBM SPSS Statistics for Windows, Version 22.0. Armonk, NY, USA).

\section{Results}

A total of 147 women who underwent CESM for a screen-detected breast abnormality were included. A total of 588 FFDM images were compared to 588 LE images. Mean age was 59.5 years (range 49-75 years). Thirty-six of 147 women were diagnosed with breast cancer $(24.5 \%)$. Of these women seven were diagnosed with DCIS (4.8\%) and the remaining 29 with invasive carcinoma (19.7\%). The remaining women were diagnosed with a benign condition as reason for their referral (such as superposition of glandular tissue as cause for the abnormality, cysts, fibro adenoma, papilloma, etc.). The mean time between FFDM at time of referral and CESM was 14 days (SD 11 days).

The results of the scores are summarized in Table 1. For FFDM images, the frequency of the maximum (' 5 ') score per criterion ranged from $40.8 \%$ to $100 \%$. For LE, this ranged from $20.1 \%$ to $100 \%$. For 19 criteria the median score for both FFDM and LE was 5 with an exception of the visibility of the pectoral muscle, where the median score of both FFDM and CESM was 4 . In 17 of the 20 image quality criteria no significant differences between FFDM and LE were observed. One criterion concerning the visibility of the pectoral muscle showed a maximal score frequency of $40.8 \%$ for FFDM and $20.1 \%$ of LE, respectively $(\mathrm{p}<0.0001)$. Of the two remaining criteria (both regarding visualization of micro calcifications) LE score was better than FFDM ( $p=0.02$ and $p=0.034$, respectively, Table 1$)$. 


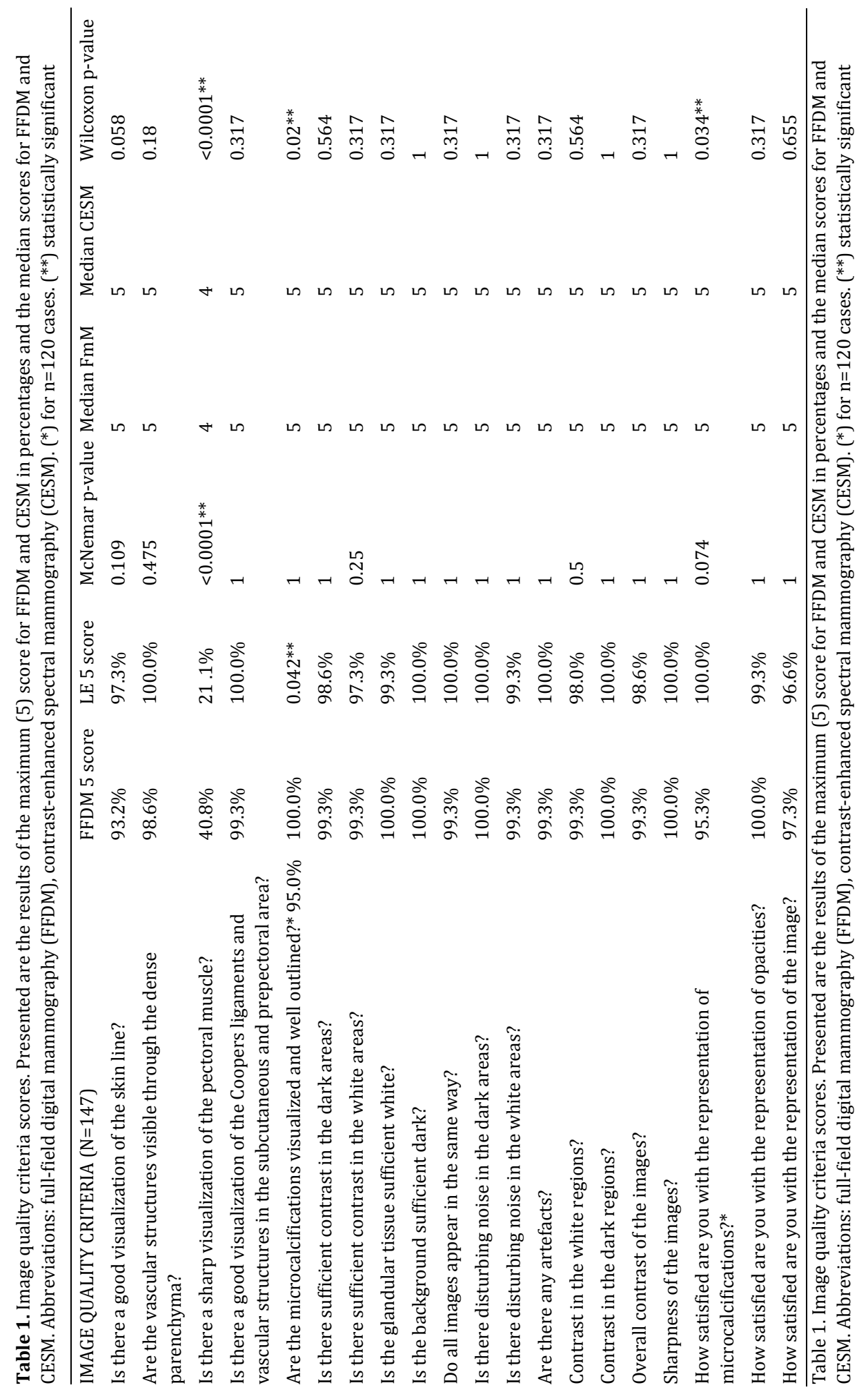


Figure 2 presents the AGD values of FFDM and LE exposures as a function of compressed breast thickness of all patients. The average pairwise difference of the AGD values was $42 \%$ where the AGD of the LE was higher than that of FFDM. Pairwise analysis showed that not only for the whole patient population but also for all breast thicknesses categories the LE AGD values were significantly higher than the FFDM AGD values (Table 3). Both LE CESM and FFDM AGD-values were in compliance with the acceptable limits of the EUREF guidelines [4]. The settings used in the CDMAM phantom measurements (Table 2) and the resulting AGD values (compare Table 2 to Figure 2) are representative for settings used in the patient groups. Furthermore, the AGD values used in all measurements are well below the achievable dose levels reported in the EUREF

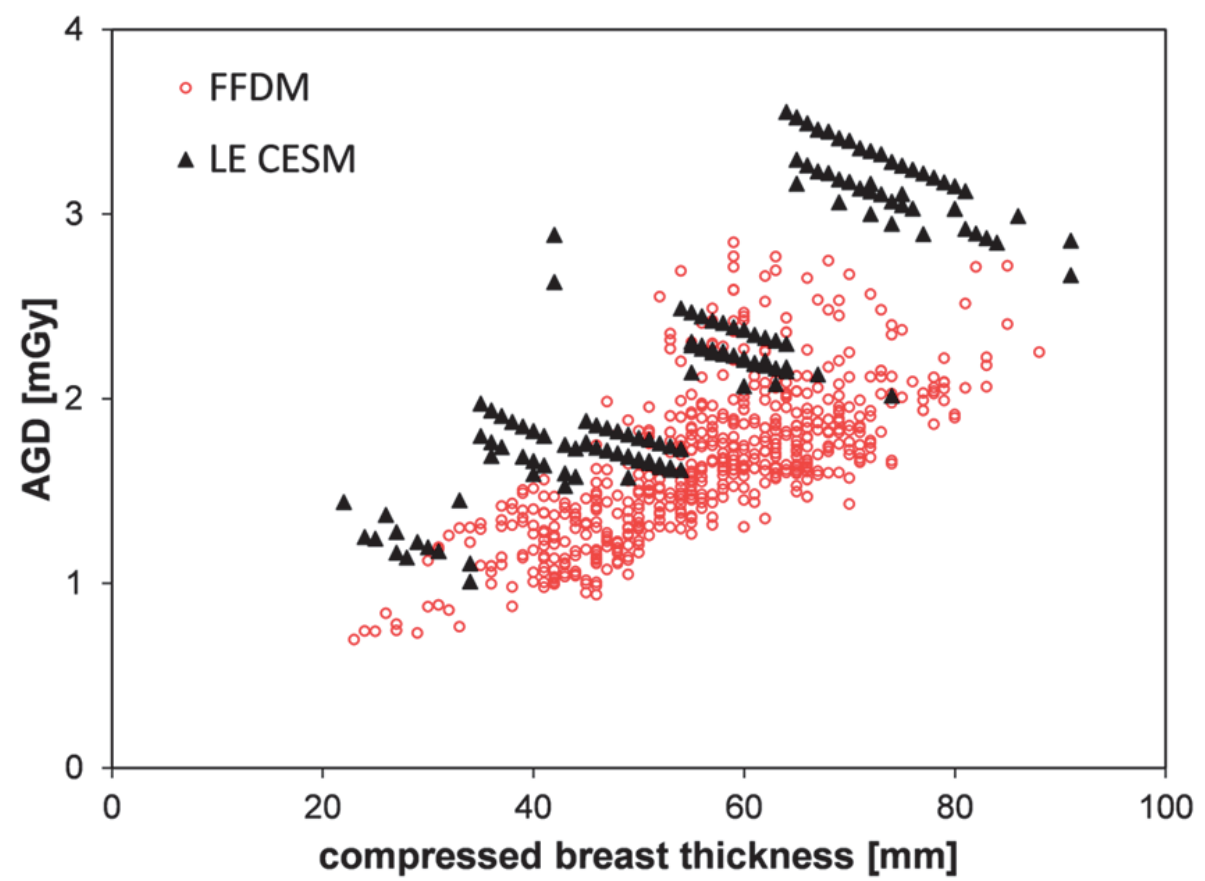

Figure 2. AGD of the complete patient group values plotted against compressed breast thickness for the FFDM (open red circles) and LE (solid black triangles) exposures. 
Table 2. Phantom composition and settings used in the CDMAM measurements.

\begin{tabular}{|c|c|c|c|c|c|c|c|c|c|c|c|c|}
\hline \multirow{4}{*}{$\begin{array}{l}\text { Equivalent } \\
\text { Breast } \\
\text { Thickness } \\
(\mathrm{mm})\end{array}$} & \multirow{4}{*}{$\begin{array}{l}\text { Total } \\
\text { PMMA } \\
\text { Thickness } \\
\text { (mm) }\end{array}$} & \multirow{4}{*}{$\begin{array}{l}\text { Phantom } \\
\text { composition* } \\
(\mathrm{mm})\end{array}$} & \multicolumn{5}{|l|}{ FFOM } & \multicolumn{5}{|c|}{ LE CESM } \\
\hline & & & \multirow{3}{*}{ Targe } & \multirow{3}{*}{ Filter } & \multirow{3}{*}{$\begin{array}{l}\text { Tube } \\
\text { Voltage } \\
(\mathrm{kV})\end{array}$} & \multirow{3}{*}{$\begin{array}{l}\text { Tube } \\
\text { load } \\
\text { (mAs) }\end{array}$} & \multirow{2}{*}{ AGD } & \multirow{2}{*}{\multicolumn{2}{|c|}{ Target Filter }} & \multirow{3}{*}{$\begin{array}{l}\text { r Tube } \\
\text { Voltage } \\
(\mathrm{kV})\end{array}$} & \multirow{3}{*}{$\begin{array}{l}\text { Tube } \\
\text { load } \\
\text { (mAs) }\end{array}$} & \multirow{3}{*}{$\begin{array}{l}\text { AGD } \\
\text { (mGy) }\end{array}$} \\
\hline & & & & & & & & & & & & \\
\hline & & & & & & & (mGy) & & & & & \\
\hline 32 & 30 & $10 / \mathrm{Ph} / 10$ & $\mathrm{~W}$ & $\mathrm{Rh}$ & 26 & 61 & 0.79 & Mo & $\mathrm{Rh}$ & 27 & 45 & 1.1 \\
\hline 45 & 40 & $10 / \mathrm{Ph} / 10 / 10$ & $\mathrm{~W}$ & $\mathrm{Rh}$ & 28 & 86 & 1.1 & $\mathrm{Rh}$ & $\mathrm{Rh}$ & 29 & 71 & 1.8 \\
\hline 60 & 50 & $10 / 10 / \mathrm{Ph} / 10 / 10$ & $\mathrm{~W}$ & $\mathrm{Rh}$ & 32 & 81 & 1.3 & $\mathrm{Rh}$ & $\mathrm{Rh}$ & 31 & 80 & 2.3 \\
\hline 75 & 60 & $25 / \mathrm{Ph} / 25$ & $\mathrm{~W}$ & $\mathrm{Ag}$ & 30 & 111 & 1.7 & $\mathrm{Rh}$ & $\mathrm{Rh}$ & 30 & 140 & 3.6 \\
\hline 90 & 70 & $25 / \mathrm{Ph} / 25 / 10$ & W & $\mathrm{Ag}$ & 34 & 96 & 1.8 & $\mathrm{Rh}$ & $\mathrm{Rh}$ & 30 & 140 & 3.8 \\
\hline
\end{tabular}

$* \mathrm{Ph}=\mathrm{CDMAM}$ phantom, 10 and 25 refers to the thickness of the PMMA plates (mm)

guidelines [4], except for one that is well below the acceptable limits. Figure 3 shows for each phantom thickness the threshold thickness of the observed gold disks versus the gold disk diameter. The threshold contrast visibility for both systems is well below the achievable limit values for a standard breast thickness of 60 mm (50 mm PMMA equivalent) stated in the EUREF guidelines [4]. For all breast thicknesses no significant differences in detection threshold thickness were found between the FFDM and LE curves (p-values of $0.29,0.10,0.13,0.42$ and 0.12 for breast thickness of $30,45,60,75$ and $90 \mathrm{~mm}$, respectively).

Table 3. AGD per exposure for FFDM and LE images for five compressed breast thickness categories and for all patients, p-values denote the significance of a pairwise comparison.

\begin{tabular}{|c|c|c|c|c|c|c|c|c|}
\hline \multirow{2}{*}{$\begin{array}{l}\text { Compressed } \\
\text { Breast Thickness } \\
(\mathrm{mm})\end{array}$} & \multirow{2}{*}{$\begin{array}{l}\text { Number } \\
\text { of Patients }\end{array}$} & \multicolumn{3}{|l|}{$\begin{array}{l}\text { FFDM } \\
\text { AGD }\end{array}$} & \multicolumn{3}{|c|}{$\begin{array}{l}\text { LE CESM } \\
\text { AGD }\end{array}$} & \multirow[b]{2}{*}{ Significance } \\
\hline & & $\begin{array}{l}\text { Mean } \\
\text { (mGy) }\end{array}$ & $\begin{array}{l} \pm \mathrm{SD} \\
\text { (mGy) }\end{array}$ & $\begin{array}{l}\text { Range } \\
\text { (mGy) }\end{array}$ & $\begin{array}{l}\text { Mean } \\
\text { (mGy) }\end{array}$ & $\begin{array}{l} \pm \mathrm{SD} \\
\text { (mGy) }\end{array}$ & $\begin{array}{l}\text { Range } \\
\text { (mGy) }\end{array}$ & \\
\hline$<40 \mathrm{~mm}$ & 13 & 1.15 & 0.24 & $0.69-1.75$ & 1.6 & 0.3 & $1.0-2.0$ & $<0.001$ \\
\hline $41-50 \mathrm{~mm}$ & 31 & 1.3 & 0.3 & $0.9-2.6$ & 1.8 & 0.1 & $1.5-2.5$ & $<0.001$ \\
\hline $51-70 \mathrm{~mm}$ & 87 & 1.8 & 0.3 & $1.1-2.8$ & 2.5 & 0.6 & $1.6-3.6$ & $<0.001$ \\
\hline $71-80 \mathrm{~mm}$ & 13 & 2.0 & 0.3 & $1.4-2.7$ & 3.22 & 0.12 & $3.03-3.52$ & $<0.001$ \\
\hline$>81 \mathrm{~mm}$ & 3 & 2.19 & 0.23 & $1.90-2.71$ & 2.95 & 0.18 & $2.67-3.26$ & $<0.001$ \\
\hline All & 147 & 1.7 & 0.4 & $0.7-2.8$ & 2.3 & 0.7 & $1.0-3.6$ & $<0.001$ \\
\hline
\end{tabular}

\section{Discussion}

CESM is a promising new breast imaging modality that has been shown to be superior to conventional mammography in the detection of breast cancer $[2,3,11]$. No studies so far compared the quality of the LE images to that of FFDM in a standardized and structured manner, using well-established EUREF image quality criteria. We demonstrated that for the majority of image quality criteria there were neither clinically relevant nor statistically significant differences between these two mo- 
dalities. Interestingly, we observed that the visualization of micro calcifications is superior in LE images. Based on additional dose and contrast detail measurements, we could not find any physical explanation for these observations.

In 17 of 20 EUREF criteria no statistical significant differences were observed, indicating that there is hardly any difference between FFDM and LE in terms of image quality.
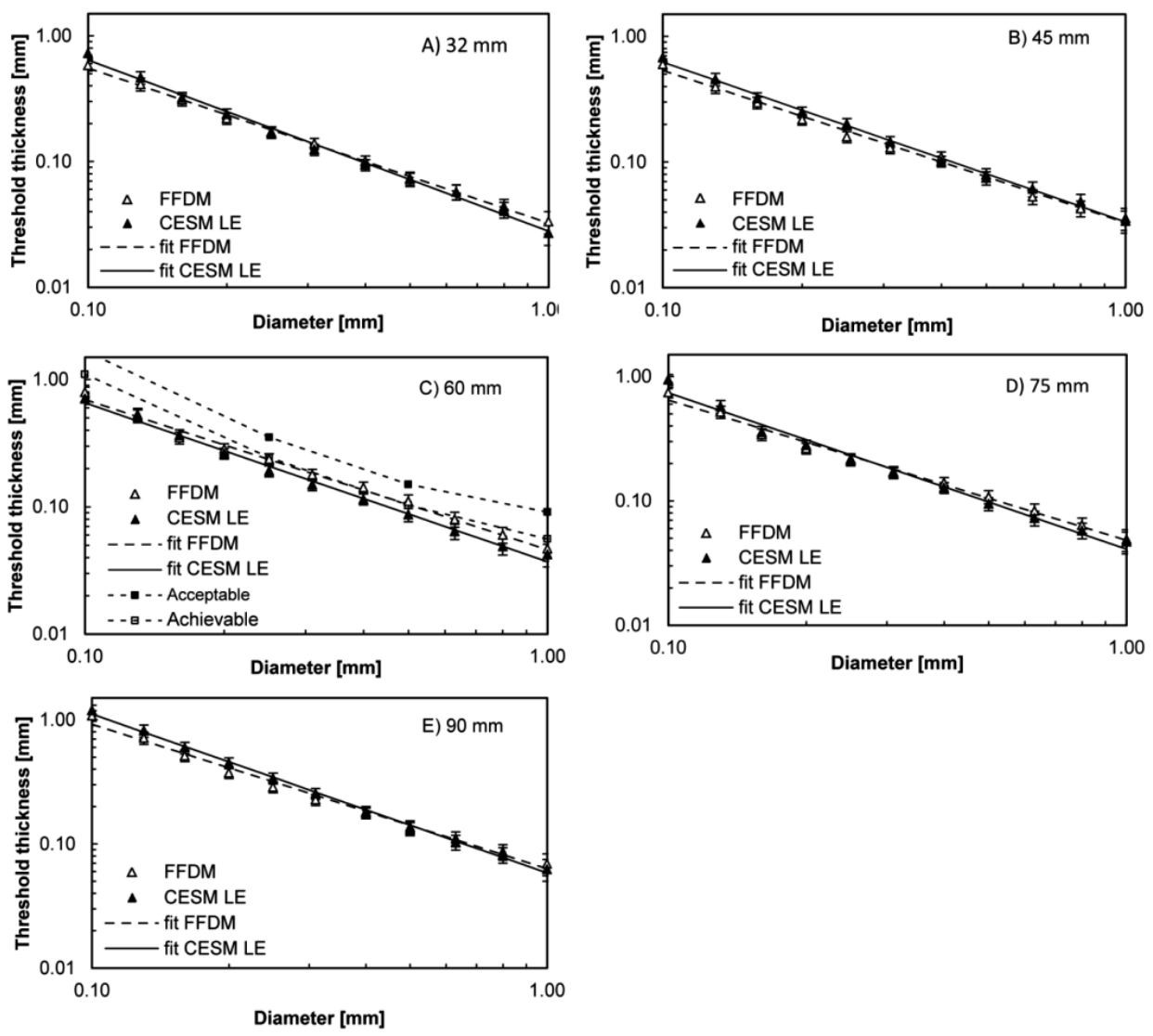

Figure 3. Threshold gold thickness detected as a function of gold disk diameter for a range of phantom thicknesses corresponding to breast thicknesses from A) $32 \mathrm{~mm}$ to E) $90 \mathrm{~mm}$. The solid and dashed lines are fits of the data. In $\mathrm{C}$ ) the acceptable and achievable limits of the EUREF Guidelines are also shown.

The delineation of the pectoral muscle was scored significantly lower for LE when compared to FFDM. However, in our experience this will not cause any relevant diagnostic dilemmas, as the recombined image can also be used for assessment of lesions covering the pectoral muscle. In a worst case scenario, an additional FFDM in mediolateral oblique view can still be considered if the lesion of interest is adjacent to the pectoral muscle. Figure 4 shows an example of the obscured pectoral 
muscle on LE versus FFDM. More interestingly, we observed a statistically significant improvement of micro calcification visualization in LE images when compared to FFDM. This latter finding can indeed be of interest, as it suggests that ductal carcinoma in situ can be diagnosed more confidently or its extent might be assessed more accurately using LE CESM images. However, the limited number of DCIS cases $(n=7)$ in this study refrained us from further testing this hypothesis.
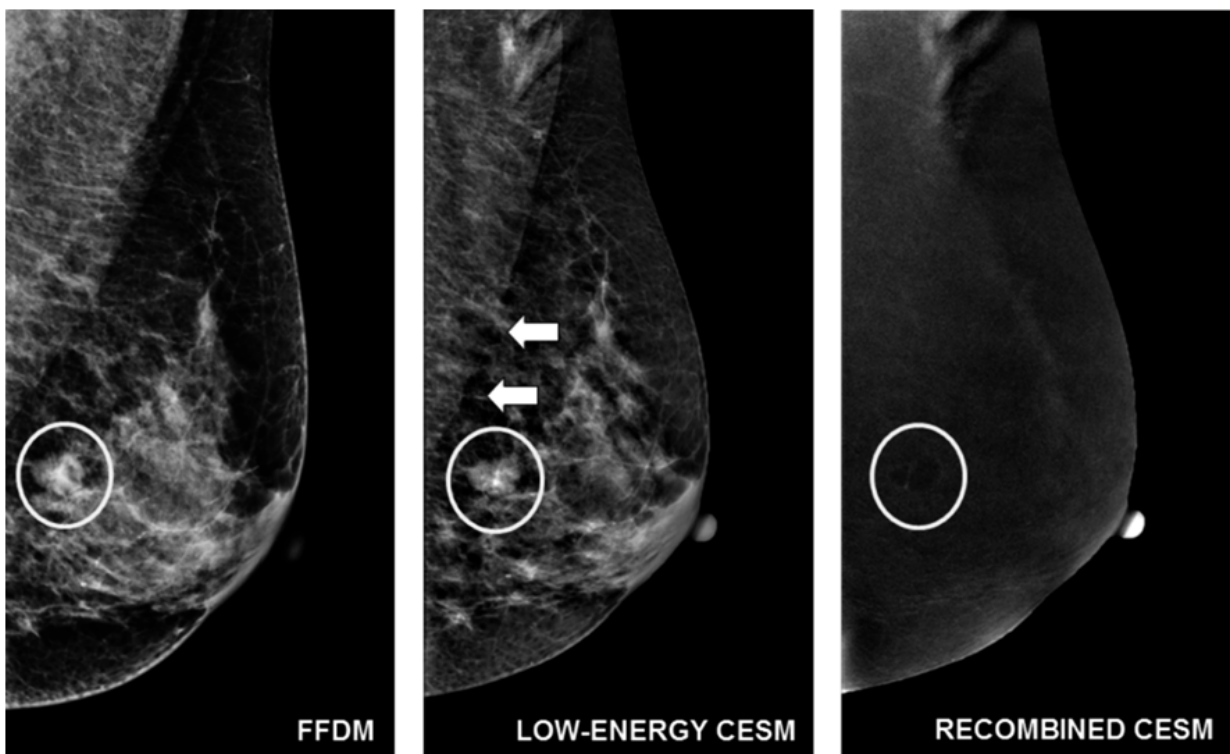

Figure 4. A 54-year-old female recalled from the breast cancer screening program (FFDM image) for a round mass in the left breast $\left(^{*}\right.$ ), also visible on the low-energy CESM image. On the recombined image, an 'eclipse sign' is visible, suggesting a cyst and confirmed by targeted ultrasound. In this case, the sharpness of the pectoral muscle was scored ' 5 ' on the FFDM image. On the low-energy CESM image, the delineation of the pectoral muscle was lost (white arrows), resulting in a score of ' 3 ' from both radiologists.

To further explain the differences in image quality scores we performed additional dose and contrast detail measurements. The average AGD of the FFDM exposure is $1.7 \pm 0.4 \mathrm{mGy}$ while the AGD of the LE exposure is $2.3 \pm 0.7 \mathrm{mGy}$. The difference reflects differences in AOP settings for FFDM and LE CESM, i.e. target/filter combination, tube voltage and tube load, where for LE CESM the prerequisite was that the LE acquisition should be suitable for reconstruction of the contrast-enhanced image. Furthermore, the dose used in these exams were in agreement with results from a previous study [12], showing that the observed image quality differences could not be explained by using abnormal radiation dose in one of the techniques. We also performed CDMAM contrast detail measurements to investigate whether the observed differences in scoring could be explained. The different scores concerning pectoral muscle visibility could not be assessed by these measurements as this structure is not represented in the phantom. The detectability of smaller diameter gold disks in this phantom was found to relate to micro calcification detec- 
tion [13]. We did not observe a significant difference in the contrast detail curves, indicating that FFDM and LE CESM have similar contrast detail detection. FFDM and LE CESM both comply with the acceptable as well as the achievable limits for contrast threshold visibility. Since the CDMAM analysis was performed on the unprocessed images, it can be concluded that the observed differences were not due to hardware differences. A possible explanation for the improved calcification detection may be found in different post-processing techniques performed to obtain the final image from the unprocessed images. Unfortunately, we are not able to further evaluate the effect of these post-processing steps in our phantom or clinical measurements.

Only two previous papers focused on the potential of LE images as replacement for FFDM. Fallenberg et al. [14] focused on the ability of cancer detection and size estimation of bilateral CESM, digital mammography, and the combination of CESM and mammography in 107 patients. They did not use image quality criteria such as those suggested by EUREF. In their study, sensitivity of mammography was $77.9 \%$, which increased to approximately 95\% when using CESM alone or the combination of CESM and mammography, while CESM alone showed the best correlation with pathology regarding tumour size measurements. Although there are minimal and hardly noticeable differences in attenuation due to the iodine contrast agent already present in the breast during the LE acquisition, the improved sensitivity was suggested to be attributed to a slight enhancement of cancers even on the LE images. In addition, our observation suggests that micro calcifications can be better visualized on LE images. Most importantly, both the study of Fallenberg and ours did not show any signs of inferiority of LE images compared to mammography.

Francescone et al. [15] studied 170 exams in 88 women and compared the LE images with FFDM images that were obtained within six months. They mainly focused on technical parameters including posterior nipple line distance, compression thickness, and compression force on the MLO projection. Similar to Fallenberg et al., they compared tumour size measurements on both modalities. An important limitation to this study was that the readers were not blinded and analysis was performed in a side-by-side comparison, thereby potentially introducing bias. No statistically significant differences were observed for all studies' parameters between LE and FFDM, but this study also did not use standardized image quality criteria such as suggested by EUREF.

Although the studies of Fallenberg and Francescone suggested non-inferiority of LE CESM compared to FFDM, our study contains important additional information as it assessed differences by using well-defined image quality criteria in a structured and standardized manner in the largest study population so far. Nevertheless, when all current evidence is summarized, it is valid and safe to conclude that LE CESM images are indeed non-inferior to FFDM, and might even be superior in terms of breast cancer detection and micro calcification evaluation. It means that FFDM can be omitted if a direct indication for CESM exists, for example in (post- 
menopausal) women with a suspicious palpable breast mass, or women with incidental suspicious breast findings observed in other imaging exams, such as chest CT exams.

Our study has some limitations. First, FFDM images were made on a different mammography unit as the LE images. Both radiologists however were experienced in reading images from both mammography units. Consequently, they could distinguish the FFDM from the LE images based on their appearance alone. However, our current (random) reading strategy, including a six-week interval, minimized the effect of any recall bias. Another limitation is that in our contrast detail phantom studies, we have used the unprocessed images for data-analysis. This is the standardized method, recommended by EUREF, as the post-processing may introduce image artefacts and may slightly differ from the mammography postprocessing as object dependent histogram of texture analysis may be used. Therefore, it was not possible to determine whether post-processing improved the contrast-detail detection, and thus, whether this would be an explanation for the significantly improved detection of micro calcifications by LE images.

\section{Conclusion}

The low-energy images obtained as part of a CESM examination are similar to FFDM for the vast majority of EUREF image quality criteria. The slightly poorer delineation of the pectoral muscle on low-energy images does not seem to cause diagnostic dilemmas. More importantly, micro calcifications are better visualized on low-energy images when compared to FFDM. In conclusion, using LE images for diagnostic purposes is safe. 


\section{References}

1. Carney PA, Miglioretti DL, Yankaskas BC, et al. (2003) Individual and combined effects of age, breast density, and hormone replacement therapy use on the accuracy of screening mammography. Ann Intern Med 138:168-75.

2. Lalji U, Lobbes M (2014) Contrast-enhanced dual-energy mammography: a promising new imaging tool in breast cancer detection. Womens Health (Lond Engl) 10:289-98. doi: 10.2217/whe.14.18

3. Lobbes MBI, Smidt ML, Houwers J, et al. (2013) Contrast enhanced mammography: techniques, current results, and potential indications. Clin Radiol 68:935-44. doi: 10.1016/j.crad.2013.04.009

4. Blum KS, Rubbert C, Mathys B, et al. (2014) Use of contrast-enhanced spectral mammography for intramammary cancer staging: preliminary results. Acad Radiol 21:1363-9. doi: 10.1016/ j.acra.2014.06.012

5. Luczyńska E, Heinze-Paluchowska S, Dyczek S, et al. (2014) Contrast-enhanced spectral mammography: comparison with conventional mammography and histopathology in 152 women. Korean J Radiol 15:689-96. doi: 10.3348/kjr.2014.15.6.689

6. (2013) European guidelines for Guality Assurance in breast cancer screening and diagnosis, Fourth edition Supplements. availible at: http://www.euref.org/european-guidelines

7. Timmers JMH, Verbeek ALM, IntHout J, et al. (2013) Breast cancer risk prediction model: a nomogram based on common mammographic screening findings. Eur Radiol 23:2413-9. doi: 10.1007/s00330-013-2836-8

8. EUREF European Guidelines - EUREF | European Reference Organisation for Quality Assured Breast Screening and Diagnostic Services. Availible at: http://www.euref.org/european-guidelines

9. Dance DR, Skinner CL, Young KC, et al. (2000) Additional factors for the estimation of mean glandular breast dose using the UK mammography dosimetry protocol. Phys Med Biol 45:3225-3240. doi: 10.1088/0031-9155/45/11/308

10. Dance DR, Young KC, van Engen RE (2009) Further factors for the estimation of mean glandular dose using the United Kingdom, European and IAEA breast dosimetry protocols. Phys Med Biol 54:4361-72. doi: 10.1088/0031-9155/54/14/002

11. Lobbes MBI, Lalji U, Houwers J, et al. (2014) Contrast-enhanced spectral mammography in patients referred from the breast cancer screening programme. Eur Radiol 24:1668-76. doi: 10.1007/s00330-014-3154-5

12. Jeukens CRLPN, Lalji UC, Meijer E, et al. (2014) Radiation Exposure of Contrast-Enhanced Spectral Mammography Compared With Full-Field Digital Mammography. Invest Radiol. doi: 10.1097/RLI.0000000000000068

13. Warren LM, Green FH, Shrestha L, et al. (2013) Validation of simulation of calcifications for observer studies in digital mammography. Phys Med Biol 58:N217-28. doi: 10.1088/0031$9155 / 58 / 16 / \mathrm{N} 217$

14. Fallenberg EM, Dromain C, Diekmann F, et al. (2014) Contrast-enhanced spectral mammography: Does mammography provide additional clinical benefits or can some radiation exposure be avoided? Breast Cancer Res Treat 146:371-81. doi: 10.1007/s10549-014-3023-6

15. Francescone MA, Jochelson MS, Dershaw DD, et al. (2014) Low energy mammogram obtained in contrast-enhanced digital mammography (CEDM) is comparable to routine full-field digital mammography (FFDM). Eur J Radiol 83:1350-5. doi: 10.1016/j.ejrad.2014.05.015 



\section{Contrast-enhanced spectral mammography in patients referred from the breast cancer screening program}

Marc B. I. Lobbes

Ulrich C. Lalji

Janneke Houwers

Estelle C. Nijssen

Patty J. Nelemans

Lori van Roozendaal

Marjolein L. Smidt

Esther Heuts

Joachim E. Wildberger

Published in: European Radiology 2014 


\section{Abstract}

Objectives: Feasibility studies have shown that contrast-enhanced spectral mammography (CESM) increases diagnostic accuracy of mammography. We studied diagnostic accuracy of CESM in patients referred from the breast cancer screening program, who have lower disease prevalence than previously published papers on CESM.

Methods: During 6 months, all women referred to our hospital were eligible for CESM. Two radiologists blinded for final diagnosis provided BI-RADS classifications for conventional mammography and CESM. Statistical significance of differences between mammography and CESM was calculated using McNemar's test. Receiver operating characteristic (ROC) curves were constructed for both imaging modalities.

Results: Of the 116 eligible women, 113 underwent CESM. CESM increased sensitivity to $100.0 \%(+3.1 \%)$, specificity to $87.7 \%(+45.7 \%)$, PPV to $76.2 \%(+36.5 \%)$, and NPV to $100.0 \%(+2.9 \%)$ as compared to mammography. Differences between conventional mammography and CESM were statistically significant $(\mathrm{p}<0.0001)$. A similar trend was observed in the ROC curve. For conventional mammography, AUC was 0.779. With CESM, AUC increased to $0.976(\mathrm{p}<0.0001)$. In addition, good agreement between tumour diameters measured using CESM, breast MRI and histopathology were observed.

Conclusion: CESM increases diagnostic performance of conventional mammography, even in lower prevalence patient populations such as referrals from breast cancer screening. 


\section{Introduction}

Mammography remains the method of choice for breast imaging, despite the development and improvement of other imaging modalities in recent decades. In the field of mammography too, significant technical improvements were realized, mainly due to the introduction of digital mammography. However, even though diagnostic accuracy of digital full field mammography is good, it depends heavily on breast density $[1,2]$. Abnormalities become more difficult to detect with increasing breast density, because of the latter's masking effects. Indeed, Carney et al. showed that sensitivity of screening mammography in extremely dense breasts was $62.9 \%$, versus $87.0 \%$ in fatty involution breasts. Specificity was $89.1 \%$ in extremely dense breasts, versus $96.9 \%$ in fatty involution breasts [2].

Full field digital mammography allows for new approaches not feasible in the analogue era, such as the use of iodine-based contrast agents. As is known from breast MRI, breast cancers show enhancement after administration of contrast agent due to the fact that tumoural micro vessels form rapidly and consequently, often have 'leaky' basement membranes. This makes the vessels permeable to contrast agent, resulting in tumour enhancement [3]. Contrast-enhanced spectral mammography (CESM) is based on this principle.

A recent summary review on contrast-enhanced mammography techniques found preliminary results encouraging [4]. The reviewed studies consisted mainly of small study populations with high breast cancer prevalence. Knowledge of diagnostic accuracy of CESM in a (clinical) population with a relatively low pre-test probability of breast cancer is currently lacking.

Our study aim was to evaluate the diagnostic performance of CESM as compared to conventional mammography in a true clinical population with the lowest disease prevalence reported thus far. For this reason, we chose to perform CESM in women referred from the Dutch breast cancer screening program for a breast abnormality, for whom breast cancer prevalence is known to be approximately $31 \%$ [5].

\section{Materials and methods}

Patients eligible for this study were women referred to our hospital from the breast cancer screening program between January 1st and July 1st, 2013. Patients with a known allergy for iodine-based contrast agents or at risk for developing contrast-induced nephropathy (as assessed by the guidelines provided by the Contrast Media Safety Committee of the European Society of Urogenital Radiology [6]) were excluded. The remaining patients were eligible to undergo CESM, the requirement for obtaining informed consent having been waived by our ethics committee.

In the Dutch screening program, women between 50-75 years undergo mammography biannually. Women are referred for further analysis to a hospital of 
their choice when a breast abnormality is detected independently by two, or in case of discrepancies three, certified screening radiologists.

\section{Standard reference procedures used to assess true disease status}

When referred to our hospital, women undergo repetition of the bilateral mammogram according to our national guideline [7]. True disease status (i.e. standard reference procedure or gold standard [8]) is assessed by one of the following strategies: if the breast abnormality is caused by superposition of normal fibroglandular tissue, at least one additional view of the ipsilateral breast next to the repeated mammogram is taken, in combination with targeted ultrasound. In agreement with the NHSBSP Clinical Guidelines for Breast Cancer Screening Assessment, women are discharged from further evaluation if all imaging is negative [9]. The safety of this approach was confirmed by an internal institutional quality control (see Discussion). If the breast abnormality is caused by cyst(s), an additional targeted ultrasound is performed to confirm the diagnosis, together with cyst aspiration to prove its non-solid state. If the breast abnormality is caused by micro calcifications or a solid mass, the repeated mammogram is combined with stereotactic or ultrasound-guided large core biopsies for histopathological diagnosis.

In some cases of breast cancer, an additional (preoperative) breast MRI is performed according to national and European indications [7, 10]. These cases enabled us to carry out a sub analysis of tumour diameter agreement using CESM, breast MRI and histopathology. All breast MRI exams were performed on a 1.5 Tesla scanner (Intera, Philips Healthcare, Best, the Netherlands) using a dedicated 16-channel breast coil. The protocol consisted of a transverse T2w sequence and diffusion weighted imaging (DWI), combined with a dynamic, contrast-enhanced T1w sequence in the transverse plane (consisting of $1.0 \mathrm{~mm}$ isotropic voxels and a temporal resolution of 93 seconds). Tumour diameter measurements were performed on the first dynamic scan after contrast administration, i.e. at peak enhancement of the tumour, using a dedicated work station (Dynacad, In vivo International, Best, the Netherlands).

\section{Imaging and image analysis}

CESM was recently introduced in our hospital as part of the breast imaging modalities. All referred cases underwent CESM (SenoBright*, GE Healthcare, United Kingdom), in which a non-ionic monomeric low-osmolar contrast agent (iopromide; Ultravist $\AA 300$, Bayer Healthcare, Germany) was administered intravenously (1.5 $\mathrm{mL} / \mathrm{kg}$ body weight, flow rate $3 \mathrm{~mL} / \mathrm{s}$, followed by saline flush). The imaging protocol of CESM itself is explained in more detail elsewhere [4, 11]. Following contrast administration, CESM acquires a set of low and high energy images in quick succession while the breast remains compressed. The low-energy image is comparable to a regular mammogram. In post-processing, both low- and high-energy 
images are used to create a so-called 'recombined image', in which contrast enhancement is visible (Figure 1).

In order to avoid influencing our standard care protocol and the indication for the performance of additional breast MRI, preliminary diagnosis was based solely on low-energy images (i.e. regular mammography). Recombined images (with enhancement information) were subsequently examined to detect additional lesions of interest.

To assess the diagnostic performance of CESM as compared to regular mammography, two dedicated breast radiologists (blinded for the final diagnosis; both viewing over 12,000 mammograms annually) retrospectively reviewed all cases by first viewing the low energy images. Similar to normal clinical practice, they were allowed to view the letter of the screening institution in which the referred breast abnormality was indicated in a drawing, together with the screening radiologists' BI-RADS classification. In this re-evaluation, the radiologists provided a BI-RADS classification for conventional mammography [12]. Subsequently, the recombined images were viewed and the radiologists were allowed to up- or downgrade their BI-RADS classification. In case of discrepancies between the two radiologists, a third breast radiologist provided consensus opinion.

\section{Statistical analysis}

BI-RADS classifications 1 to 3 were considered 'benign', and 4 and 5 'malignant'. Based on this cut-off value, sensitivity, specificity, positive predictive value (PPV), and negative predictive value (NPV) were calculated for regular mammography and CESM. Differences in diagnostic parameters between regular mammography and CESM were calculated with corresponding 95\% confidence intervals (CI) for paired proportions. The differences were tested for statistical significance using McNemar's test for paired proportions. Receiver operating characteristic (ROC) curves were constructed for both imaging modalities and the areas under the curve (AUC) with corresponding 95\% CI were calculated and compared according to the method described by Hanley et al. $[8,13]$.

Agreement between maximum lesion diameter based on CESM and MRI, or CESM and surgical specimen were expressed in Bland-Altman plots for a subgroup of patients [14]. Measurement of maximum lesion diameter was performed on the entire CESM exam (i.e. viewing low and high energy images together) or on the first post-contrast dynamic scan on MRI (approximately 93 seconds after contrast administration). For the comparison of maximum lesion diameter found using CESM and pathology, surrounding ductal carcinoma in situ (DCIS) was included in the final maximum diameter measurement. In case of multifocal breast cancer, the maximum diameter of the largest invasive tumour (i.e. primary index tumour) was assessed. Patients that underwent neoadjuvant chemotherapy $(n=3)$ were not included in the comparison with histopathological results, since good or pathological complete response might render the latter inaccurate. 

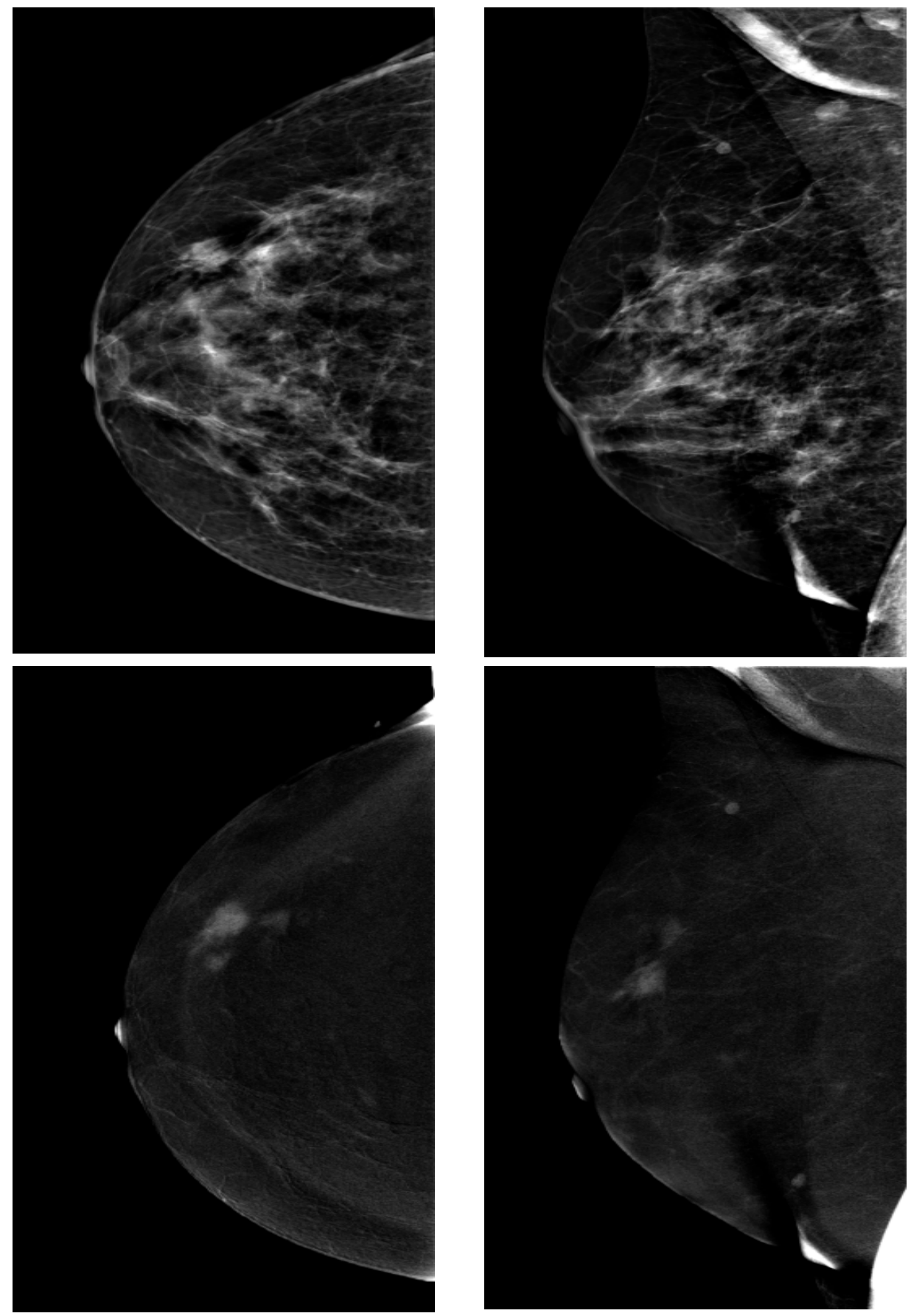

Figure 1. Typical example of CESM, showing low energy images in the top row (i.e. regular mammography) and the recombined images (showing enhancement of lesions) in the bottom row. In this case, a 54 year old patient was referred due to a new irregular density in the craniocaudal view of the right breast (arrow, top row). CESM demonstrated true extent of the disease as well as its multifocal nature of this breast cancer (bottom row), which was confirmed by histopathological analysis of the surgical specimen. 
All statistical analyses were performed using SPSS Statistics (version 20.0, IBM, Armonk, USA), STATA (StataCorp LP, College Station, USA), and MedCalc (version 12.7.8, MedCalc Software, Ostend, Belgium). P-values $<0.05$ were considered statistically significant.

\section{Results}

In our study period, 7703 women were invited to participate in the regional breast cancer screening program and 6384 women attended (82.9\%) (Figure 2). From these, a total number of 116 women (1.8\%) were referred to our hospital. One patient refused contrast administration. Two patients did not undergo CESM due to: $(n=1)$ known allergy to an iodine-based contrast agent, and $(n=1)$ renal insufficiency (estimated glomerular filtration rate of $33 \mathrm{~mL} / \mathrm{min} / 1.73 \mathrm{~m} 2$ ). Consequently, 98.3\% (113/115) of eligible patients underwent CESM (median age 54.0 years, mean 57.2 years, range $49-73$ years). Only one minor reaction to contrast administration was observed in the form of few urticarias which resolved spontaneously.

Masses or densities were the most frequent cause of referrals (76.1\%), followed by micro calcifications (15.0\%), asymmetry (6.2\%), and architectural distortions $(2.7 \%)$. The standard reference procedures resulted in the following final diagnoses: superposition densities (24.8\%), simple cysts (21.2\%), invasive ductal carcinoma (20.4\%), fibroadenoma (7.1\%), invasive lobular carcinoma $(4.4 \%)$, ductal carcinoma in situ (3.5\%, all grade 2 or 3$)$, and other benign causes $(18.6 \%)$. Breast cancer prevalence was $28.3 \%$. A detailed overview of patient characteristics is presented in Table 1.

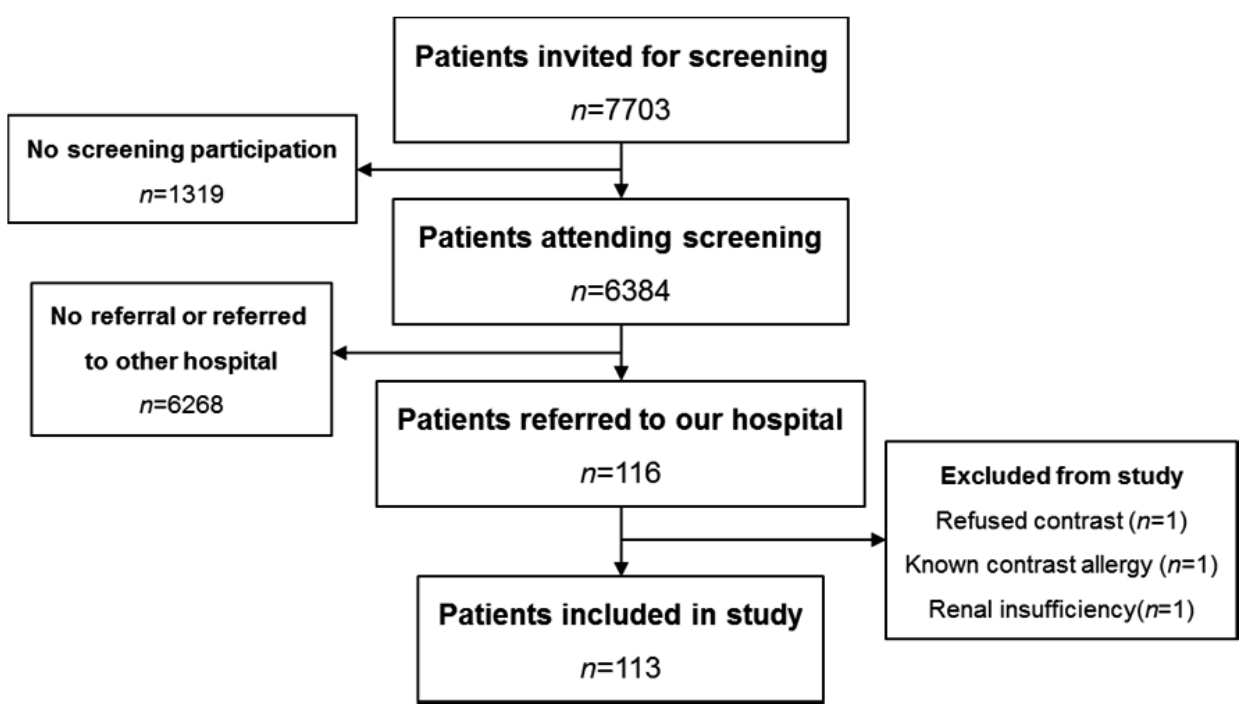

Figure 2. Flowchart of patient inclusion 
Diagnostic accuracy parameters for conventional mammography were (absolute numbers given in parentheses): sensitivity 96.9\% (31/32), specificity $42.0 \%$ (34/81), PPV 39.7\% (31/78) and NPV 97.1\% (34/35). After subsequent CESM, diagnostic accuracy parameters changed to: sensitivity $100.0 \%(32 / 32)$, specificity 87.7\% (71/81), PPV 76.2\% (32/42) and NPV 100.0\% (71/71). 95\% confidence intervals are summarized in Table 2 . In short, sensitivity remained stable when using CESM, but interestingly, specificity increased by a factor of more than 2 . The observed differences were statistically significant (Figure 3, p<0.0001). A similar trend was observed in the ROC curve: for conventional mammography, the AUC was 0.779: for CESM, the AUC increased to 0.976 (Table 2 and Figure 4, p<0.0001). Analysis of agreement between CESM and breast MRI was possible for 24 breast cancer cases. For the assessment of agreement between CESM and histopathology 30 cases were available. Bland-Altman plots showed a mean difference of $1.0 \mathrm{~mm}$ with $95 \%$ limits of agreement of $8.3 \mathrm{~mm}$ in tumour diameter measured using CESM and breast MRI. A mean difference of $1.4 \mathrm{~mm}$ was observed between tumour diameters assessed using CESM and histopathology, but with larger 95\% limits of agreement: $25.0 \mathrm{~mm}$ (Figure 5).

In this population, we coincidently encountered five false-positives based on CESM (enhancement) information only: low energy images did not show any suspicious lesions, whereas recombined CESM images showed an enhanced lesion (Figure 6). In all five cases, a solid mass was the cause of enhancement, and subsequent biopsy showed four fibroadenomas and one hamartoma.

Table 1. Patient characteristics and final diagnosis as assessed by standard reference procedures. Abbreviations: oestrogen receptor (ER), progesterone receptor (PR), human epidermal growth factor receptor 2 (HER2/neu). ( ${ }^{*}$ ) Old hematoma: caused by recent vehicle accident and seat belt injury, confirmed by tissue sampling. Tumour grade according to Bloom and Richardson.

\begin{tabular}{ll}
\hline AGE & \\
Mean & 57.2 years \\
Median & 54.0 years \\
Standard deviation & 8.0 years \\
Range & $49-73$ years \\
FINAL DIAGNOSIS & \\
Superposition density & $24.8 \%$ \\
Simple cyst & $21.2 \%$ \\
Invasive ductal carcinoma & $20.4 \%$ \\
Fibroadenoma & $7.1 \%$ \\
Apocrine metaplasia & $5.3 \%$ \\
Invasive lobular carcinoma & $4.4 \%$ \\
Ductal carcinoma in situ & $3.5 \%$ \\
Cylindrical cell changes & $3.5 \%$ \\
Papilloma & $1.8 \%$ \\
Intramammary lymph node & $1.8 \%$ \\
\hline
\end{tabular}




\begin{tabular}{ll}
\hline Reactive changes & $0.9 \%$ \\
Fibrosis & $0.9 \%$ \\
Inflammatory changes & $0.9 \%$ \\
Old hematoma & $0.9 \% *$ \\
Sclerosing adenosis & $0.9 \%$ \\
Atypical lobular hyperplasia & $0.9 \%$ \\
Duct ectasia & $0.9 \%$ \\
INVASIVE BREAST CANCER SUBTYPES & \\
ER positive & $75 \%$ \\
PR positive & $64.3 \%$ \\
HER2/neu positive & $14.3 \%$ \\
Grade 1 & $7.4 \%$ \\
Grade 2 & $59.3 \%$ \\
Grade 3 & $33.3 \%$ \\
\hline
\end{tabular}

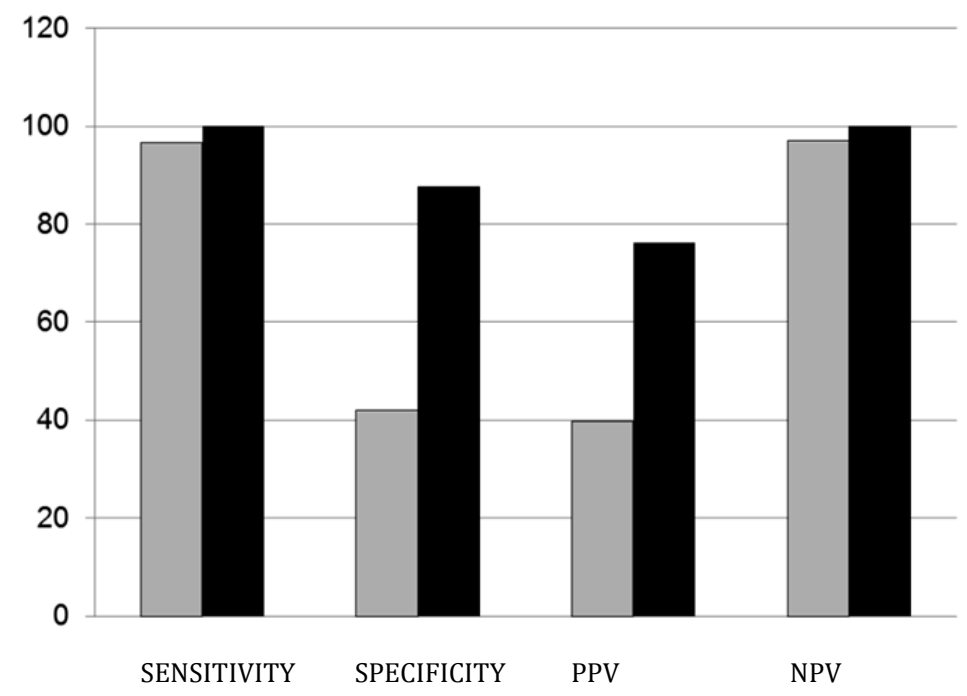

Figure 3. Diagnostic parameters for conventional mammography (grey bars) and CESM (black bars).

\section{Discussion}

Contrast-enhanced spectral mammography (CESM) is a new imaging technique, which uses mammography in combination with iodine-based contrast agents to increase diagnostic performance. A recent review on contrast-enhanced mammography techniques found encouraging preliminary results, with a mean sensitivity of $85.2 \%$ (range $62.0-96.0 \%$ ), and a mean specificity of $66.1 \%$ (range $50.0-83.3 \%$ ) [4]. These studies, consisting mainly of small study populations (mean size of 47 patients, range 20-120), were enriched with breast cancer cases (mean disease prevalence was $64.5 \%$, range $37.5-100.0 \%$ ), whereas breast cancer pre-test prob- 
ability in routine clinical practice is much lower (e.g. patients referred from the national breast cancer screening program). Evaluating diagnostic performance of CESM in a clinical population with lower breast cancer pre-test probability than previously published is the next logical step in the evaluation of CESM's imaging efficacy. To our knowledge, ours is the first study to do so.

Table 2. Overview of the diagnostic performance of mammography versus contrast-enhanced spectral mammography (CESM). 95\% confidence intervals are in parentheses.

\begin{tabular}{lll}
\hline & Mammography & CESM \\
\hline Sensitivity & $96.9 \%(83.7-99.5 \%)$ & $100.0 \%(89.0-100.0 \%)$ \\
Specificity & $42.0 \%(31.1-53.5 \%)$ & $87.7 \%(78.5-93.9 \%)$ \\
Positive predictive value & $39.7 \%(28.8-51.5 \%)$ & $76.2 \%(60.6-87.9 \%)$ \\
Negative predictive value & $97.1 \%(85.0-99.5 \%)$ & $100.0 \%(94.9-100.0 \%)$ \\
Area under the ROC curve & $0.779(0.707-0.851)$ & $0.976(0.954-0.999)$ \\
\hline
\end{tabular}

CESM is logistically feasible in clinical practice, as is demonstrated by the high percentage of eligible patients who underwent CESM (98\%). In addition, our study population was not enriched with cancer cases, reflecting a true clinical population. Breast cancer population prevalence was $28 \%$, which is in accordance with previously published findings (31\%) [5], and much lower than previously published studies (mean 64.\%, lower limit 37.5\%) [4]. We found the diagnostic accuracy of CESM to be excellent and superior to conventional mammography alone. Although sensitivity and NPV were found to be high for conventional mammography, both reached $100 \%$ upon using CESM. More importantly, specificity and PPV were found to be low for conventional mammography, and to significantly increase upon using CESM.

The increased sensitivity of mammography due to CESM was recently demonstrated by Jochelson et al. In their study, sensitivity of mammography was increased from $81 \%$ to $96 \%$ due to CESM [15]. Similar observations were recently published by Fallenberg et al., who showed that mammography's sensitivity (82.5\%) increased to $100.0 \%$ due to CESM [16]. The increased specificity found in our study underscores the contribution CESM may make towards eliminating the false-positives observed in conventional mammography. The NPV of $100 \%$ found in this study population suggests that a negative CESM exam can rule out breast cancer. 


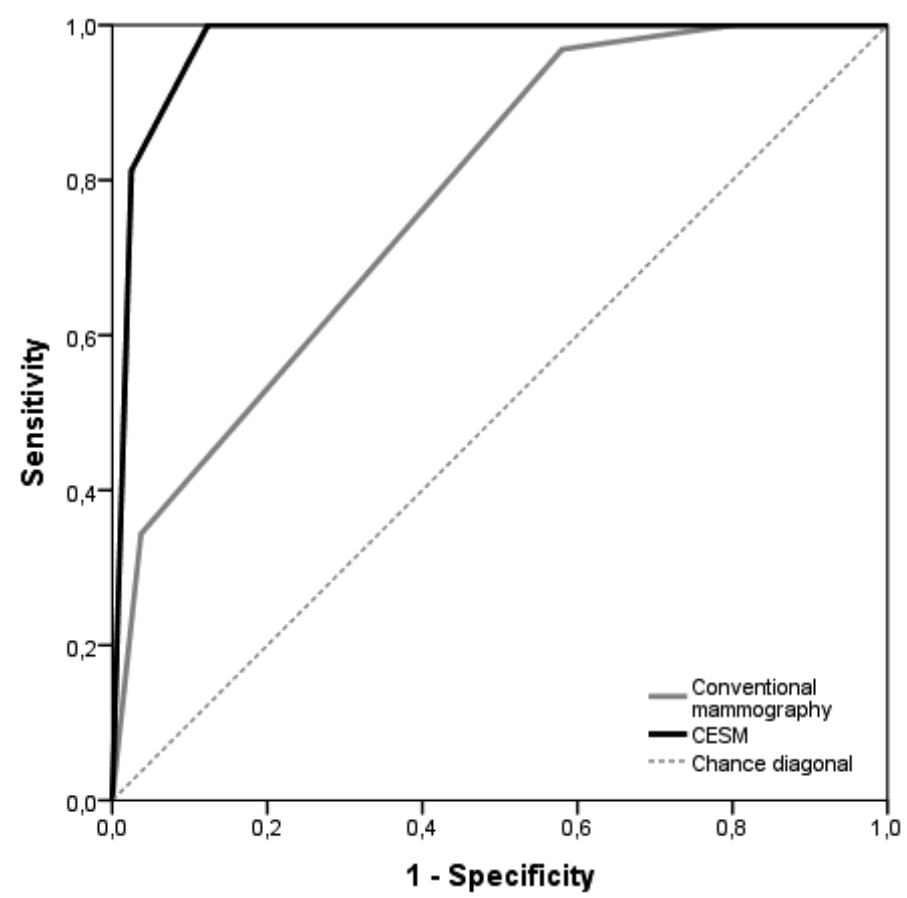

Figure 4. Receiver operating characteristic (ROC) curve for both conventional mammography (grey line) and CESM (black line).

Whilst both conventional mammography and CESM have excellent cancer detection scores, CESM may be recommended even if breast cancer is highly suspected from the screening mammography. CESM might theoretically replace breast MRI in preoperative evaluation of breast cancer patients, both techniques being based on the same principle. Although still under debate, preoperative evaluation of disease extent is one of the most frequent indications for breast MRI [12]. Jochelson et al. were the first to compare CESM with MRI in a study of 52 index tumours and found that CESM and MRI were equally accurate in the detection of breast cancer, detecting $96 \%$ of tumours [15]. A well-known limitation of breast MRI is the occurrence of false-positives, and indeed 13 false-positives were observed in the MRI exams, against only two false-positives using CESM. On the other hand, breast MRI proved to be more accurate in detecting ipsilateral additional foci of tumour cells: $88 \%$ of additional foci were detected using breast MRI, against only 56\% using CESM. The number of multifocal breast cancer cases in our population was too small to study reproducibility of these findings 

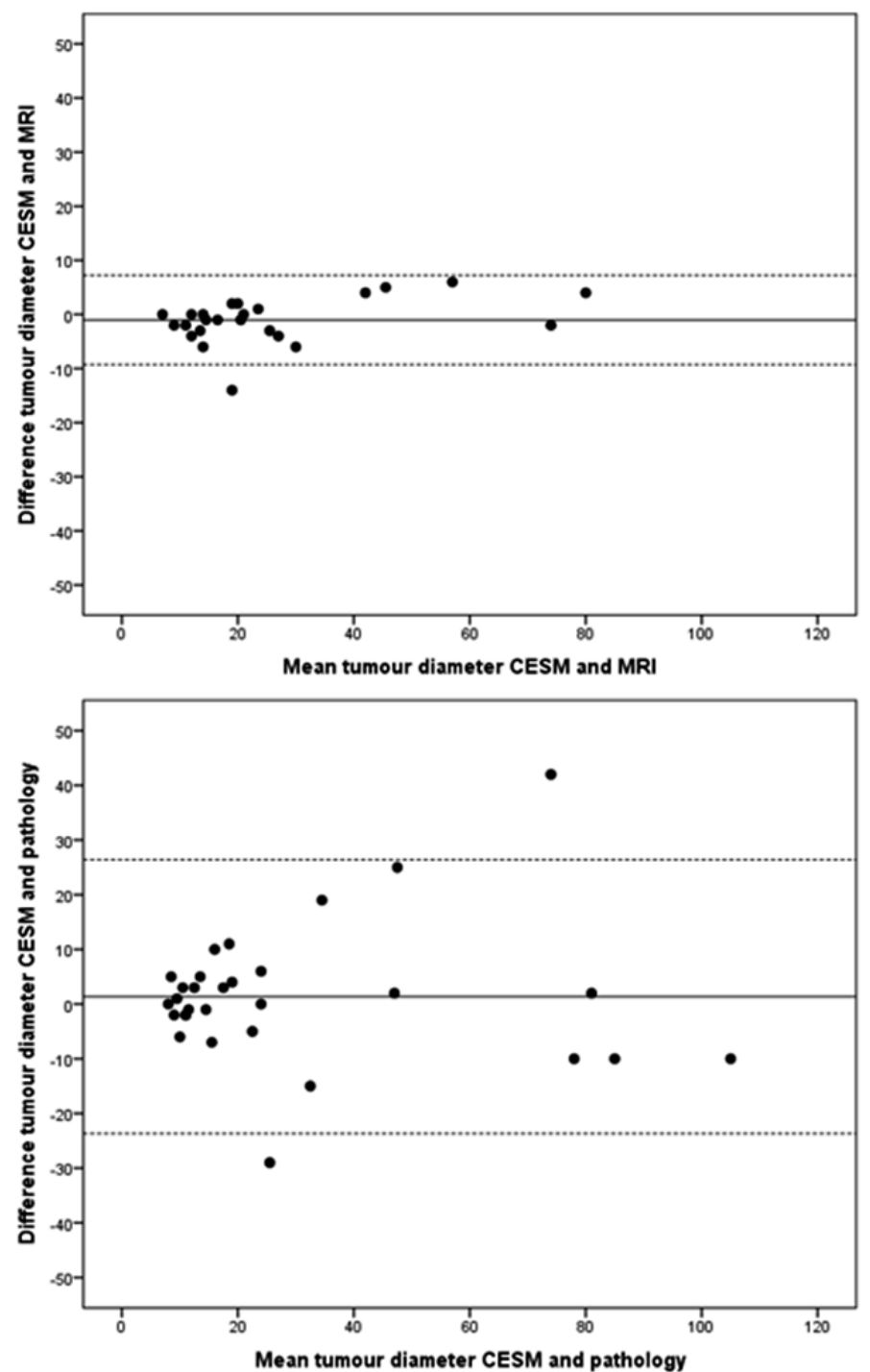

Figure 5. Bland-Altman plots of agreements between (A) tumour diameter measured using CESM and breast MRI, and (B) CESM and pathological examination. Solid lines represent the mean of the differences between measurements, dotted lines represent upper and lower limits of 1.96 times standard deviations of differences.

Recently, Fallenberg et al. confirmed the potential of CESM as replacement or alternative for breast MRI in preoperative assessment of disease extent [16]. In their study, tumour diameter as measured on mammography, CESM, MRI and histopathology were compared. The Pearson's correlation coefficient (PCC) between CESM and MRI was highly significant: $0.943, p=0.000$. However, good correlation does not automatically imply good correlation between two measurements [17]. If there is a systematic over- or underestimation between measurements, PCC can be very high, but agreement is low. Therefore, analysis of agreement between two 
measurements, for example by using the Bland-Altman plots presented in this study, is recommended in the discussion on whether CESM can replace breast MRI for tumour size measurements.

As opposed to the studies of Jochelson et al. (who presented a descriptive analysis of their findings) and Fallenberg et al. (who studied only correlation between tumour size measurements), we studied maximum tumour diameter agreement measured using CESM and breast MRI. We found a good agreement between CESM and breast MRI based measurements (Figure 5).

In addition, we studied maximum tumour diameter agreement as measured using CESM and pathology. Although the agreement found was slightly less than that found for the CESM and breast MRI based measurement, it was still considered good (Figure 5). To the best of our knowledge, only three other studies evaluated these measurements. In the first, a pilot study, Dromain et al. evaluated 20 histologically proven breast cancers and concluded that correlation between tumour diameters assessed using contrast-enhanced mammography and using histopathological results was good: $r 2=0.743$ [18]. In the second study, Dromain et al. evaluated 142 breast lesions, assessing mean lesion size with mammography, ultrasound, and a combination of mammography and CESM [19]. Mean differences between maximum tumour diameters found using these imaging techniques as compared to pathology were $1.3 \mathrm{~mm}, 5.3 \mathrm{~mm}$, and $0.7 \mathrm{~mm}$, respectively. The third study by Fallenberg et al. consisted of 80 patients with newly diagnosed breast cancer. The PCC between CESM and histopathology was statistically significant: 0.733, $p=0.000$ [16]. We observed a mean difference between CESM and pathology of only $1.4 \mathrm{~mm}$.

Breast cancer screening programs suffer from an important number of falsepositive findings. The introduction of digital breast tomosynthesis might be able to increase cancer detection while decreasing the number of false-positive referrals, as was demonstrated recently by Skaane et al. [20].

Nonetheless, women that are still referred need a reliable diagnostic work-up to establish their final diagnosis. Zuley et al. showed that digital breast tomosynthesis might play an important role in this work-up, since they showed that it significantly improved the diagnostic accuracy of non-calcified lesions when compared with supplemental mammography views [21]. Michell et al. recently showed that in women referred from a breast cancer screening program digital breast tomosynthesis increased the diagnostic accuracy of the exam when compared to mammography [22]. In their study, AUC-values were significantly higher with the addition of digital breast tomosynthesis combined with FFDM and film-screen mammography (AUC 0.967) when compared to FFDM plus film-screen mammography (AUC 0.895) and film-screen mammography alone (AUC 


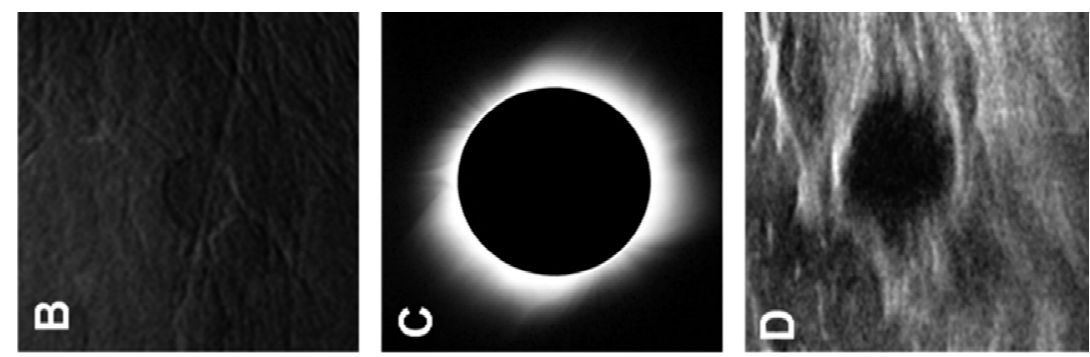

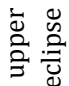

3ั 츤

त⿱ के

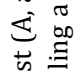

苋

웜

范

竞
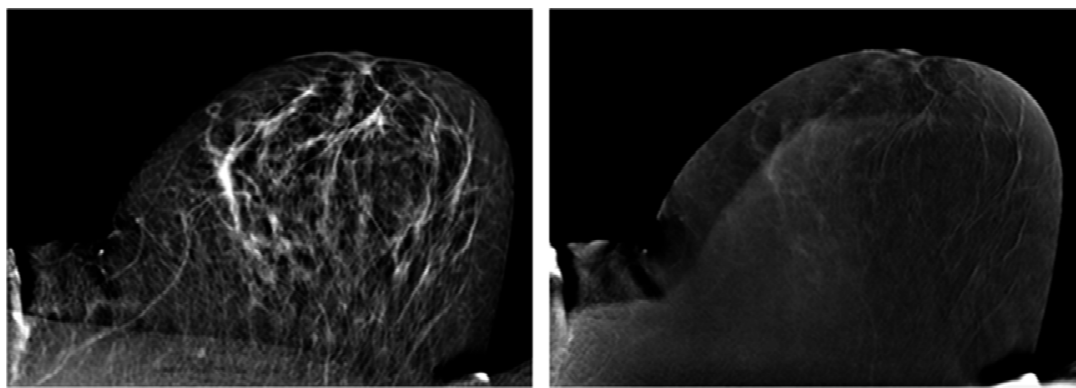

范

究 $\frac{7}{3}$

घ

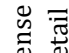

这

ฮें

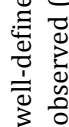
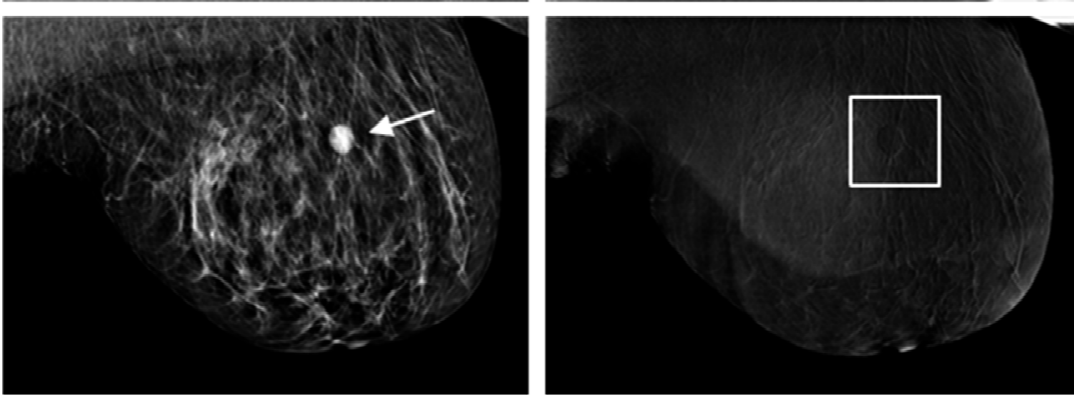

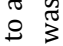

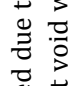

률

巳

巳

馬

龸

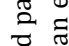

등

苋

듀
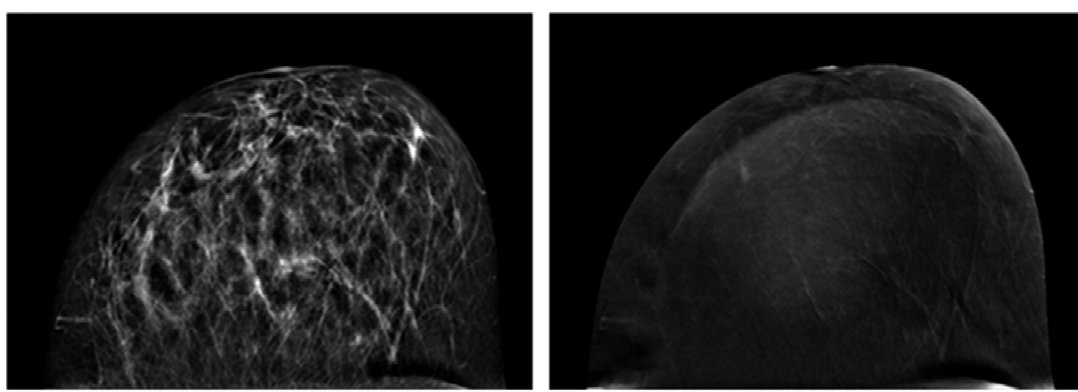

유

$\Xi \circlearrowright$

को

$\stackrel{0}{\Xi} \sum^{0} 0$

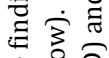

¿

물

足

$=8$.

考

品

을
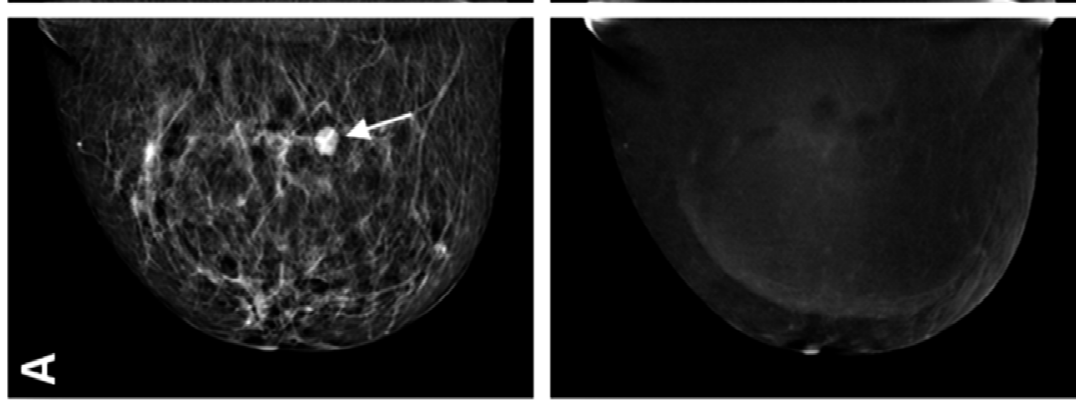

峁

0

范

匹

$\leadsto$ है

हั

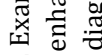

ง 을

z

范主 

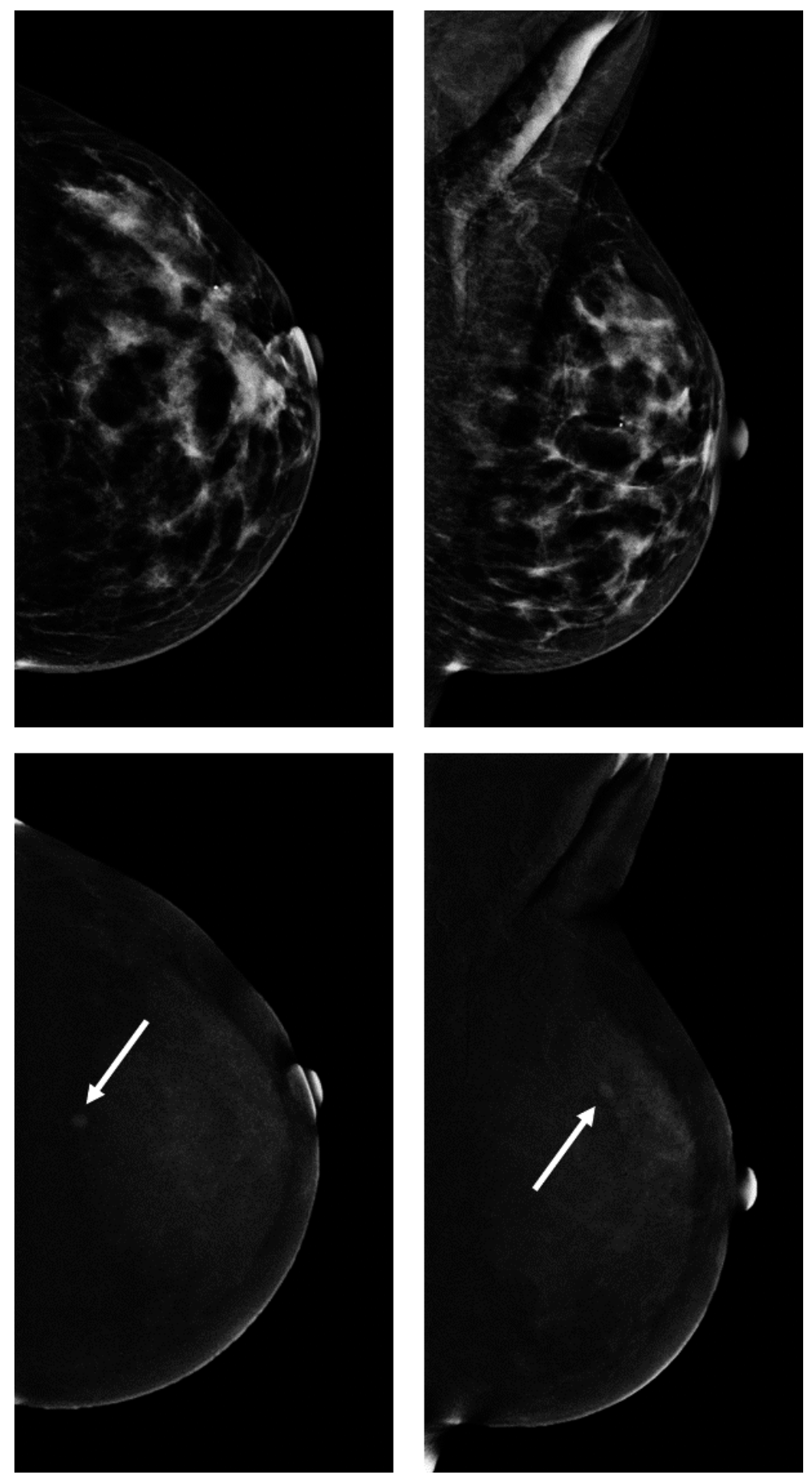

Figure 7. False positive finding due to CESM use. Top row shows low-energy images, bottom row shows recombined images (only left side shown). No abnormalities were detected on the low energy images. However, a small enhancing round mass was detected only on the recombined images (arrows). Biopsy results showed fibroadenoma. 
0.788). Although these numbers are in line with our current observations using CESM, we are of the opinion that CESM might be the more suitable technique (despite the use of intravenous contrast agent and a higher dose), since its accuracy reached $100 \%$ for 113 cases and since it might be an alternative for the entire conventional work-up, in contrast to digital breast tomosynthesis, which is only an alternative for the mammographic work-up. For example, tomosynthesis would be highly suitable to rule out superposition densities in false-positive referrals, but many masses would still require further evaluation using ultrasound. As shown in our study, CESM has the potential to rule out superposition densities as good as tomosynthesis, but it has the additional benefit that many masses caused by cysts can also be safely ruled out without the need for further imaging. In superposition densities, no enhancement whatsoever occurs in the region of interest, and in cysts a specific 'void' is seen, which strongly resembles a solar eclipse (Figure 7). We hereby suggest the introduction of the term 'eclipse' for this medical sign of cysts.

CESM also suffers from some disadvantages, of which an increase in breast radiation dose is probably the most important one. Badr et al. recently showed that compared to full-field digital mammography, CESM dose increased by $54 \%$ to 2.65 mGy per image [23]. This increase in dose should be taken into account when considering the benefits that CESM would have over conventional mammography, especially in young women. In addition, CESM will result in higher costs, since contrast is used and more time is needed to perform and read the exams. Future studies should also focus on the cost-effectiveness of this new technique.

This study has some limitations. First, our sample size was limited to 113 women in the final analysis and consisted of a population with disease prevalence lower than published before. However, the population is pre-selected at the referral stage from the screening program. Consequently, it is not possible to investigate how CESM would have performed in cases that were not included in the study population. Different screening radiologist might have resulted in different referrals, resulting in a different study population. The limited number of women might suggest that these study results are underpowered. Underpowered studies could have two important consequences: (1) undetected effects of practical importance, and (2) large variance of the parameter being measured. Even in this limited sample size, however, results were statistically significant to a high degree and therefore extremely unlikely to be based on chance. On the other hand, statistical significance does not contain information on the true differences between imaging modalities, and increasing the sample size would improve results with respect to approaching the 'true' effect of the parameters studied, and decrease variance. Furthermore, the number of breast cancer cases is small (particularly in situ cancers). Since sensitivity and specificity are correlated, this small number of abnormal cases might also influence specificity. For future studies, it would be recommendable to use a larger sample size, preferably evaluated by multiple readers.

A second potential limitation is that we were not able to assess breast density. As mentioned before, breast density can influence mammography's diagnostic 
accuracy. However, current commercially available (semi)automated breast density analysis software tools have not been validated for the use in advanced mammography techniques, such as CESM. Visual inspection of breast density is inaccurate and observer-dependent [24]. Consequently, we could not perform a reliable analysis of breast density for this study.

A third potential study limitation is that no follow-up occurred of patients with abnormalities presumably caused by superposition of fibroglandular tissue. A follow-up period of at least two years would be preferable in order to rule out any malignancies occurring in the time frame between screening rounds. However, our standard reference procedure of diagnosing superposition densities is in line with the NHSBSP Clinical Guidelines for Breast Cancer Screening Assessment. Furthermore, a recently performed institutional quality control of this approach showed that this approach can be safely used to rule out breast cancer (Van Roozendaal et al., internal publication). In this institutional quality control, 582 referrals from breast cancer screening were analysed. Of these, 95 were diagnosed as 'superposition cases' with the before mentioned approach. Follow-up results were collected for these cases, divided into groups according to their follow-up policy: single breast MRI (37 cases), mammographic follow-up in 6 (41 cases) or in 12 months (4 cases), or no follow-up (13 cases). None of the superposition lesions proved to be malignant (median follow-up 26 months).

\section{Conclusion}

CESM is logistically feasible as a breast imaging tool in clinical practice. CESM significantly increases diagnostic accuracy in patients referred from breast cancer screening as compared to conventional mammography. Our study showed that diagnostic accuracy of CESM is high, even in populations with lower pre-test breast cancer probability than previously published. The good agreement found between tumour diameters based on CESM, MRI and pathology supports CESM as potential replacement for breast MRI in preoperative evaluation of breast cancer patients. 


\section{References}

1. Pisano ED, Gatsonis C, Hendrick E, et al (2005). Diagnostic performance of digital versus film mammography for breast cancer screening. N Eng J Med 353:1773-1783.

2. Carney PA, Miglioretti DL, Yankaskas BC, et al (2003). Individual and combined effects of age, breast density, and hormone replacement therapy use on the accuracy of screening mammography. Ann Intern Med 138:168-175.

3. Kuhl C (2007). The current status of breast MR imaging part I: Choice of technique, image interpretation, diagnostic accuracy, and transfer to clinical practice. Radiology 244:356-378.

4. Lobbes MBI, Smidt ML, Houwers J, Tjan-Heijnen VC, Wildberger JE (2013). Contrast-enhanced mammography: techniques, current results and potential indications. Clin Radiol 68:935-944.

5. Timmers JMH, Van Doorne-Nagtegaal HJ, Zonderland HM, et al (2012). The Breast Imaging Reporting and Data System (BI-RADS) in the Dutch breast cancer screening programme: its role as an assessment and stratification tool. Eur Radiol 22:1717-1723.

6. Stacul F, Van der Molen AJ, Reimer P, et al (2011). Contrast-induced nephropathy: updated ESUR Contrast Media Safety Committee guidelines. Eur Radiol 21:2527-2541.

7. Nationaal Borstkanker Overleg Nederland (NABON). National guideline Breast Cancer 2012, Amsterdam, NABON, 2012.

8. Obuchowksi NA (2003). Receiver operating characteristic curves and their use in radiology. Radiology 229:3-8.

9. Mann RM, Kuhl CK, Kinkel K, Boetes C (2008). Breast MRI: guidelines from the European Society of Breast Imaging. Eur Radiol 18:1307-1318.

10. Liston J, Wilson R (2010). NHSBSP Clinical Guidelines for Breast Cancer Screening Assessment, 3rd edition. NHS Cancer Screening Programms, Sheffield, United Kingdom.

11. Dromain C, Balleyguier C, Adler G, Garbay JR, Delaloge S (2009). Contrast-enhanced digital mammography. Eur J Radiol 69:34-42.

12. American College of Radiology (2003). Breast Imaging Reporting and Data System (BI-RADS), volume 4, American College of Radiology, Reston, USA.

13. Hanley JA, McNeil BJ (1983). A method of comparing the area under receiver operating characteristic curves derived from the same cases. Radiology 148:839-843.

14. Bland JM, Altman DG (1986). Statistical methods for assessing agreement between two methods of clinical measurement. Lancet 1:307-310.

15. Jochelson MS, Dershaw DD, Sung JS, et al (2013). Bilateral contrast-enhanced dual energy digital mammography: feasibility and comparison with conventional digital mammography and MR imaging in women with known breast carcinoma. Radiology 266:743-751.

16. Fallenberg EM, Dromain C, Diekmann F, et al (2014). Contrast-enhanced spectral mammography versus MRI: Initial results in the detection of breast cancer and assessment of tumour size. Eur Radiol 24:256-264.

17. Lobbes MB, Nelemans PJ (2013). Good correlation does not automatically imply good agreement: The trouble with comparing tumour size by breast MRI versus histopathology. Eur J Radiol 82:e906-907.

18. Dromain C, Balleyguier C, Muller S, et al (2006). Evaluation of tumour angiogenesis of breast carcinoma using contrast-enhanced digital mammography. Am J Roentgenol 187:528-537.

19. Dromain C, Thibault F, Muller S, et al (2011). Dual-energy contrast-enhanced digital mammography: initial clinical results. Eur Radiol 21:565-574.

20. Skaane P, Bandos AI, Gullien R, et al (2013). Comparison of digital mammography alone and digital mammography plus tomosynthesis in a population-based screening program. Radiology 267:47-56.

21. Zuley ML, Bandos AI, Ganott MA, et al (2013). Digital breast tomosynthesis versus supplemental diagnostic mammographic views for evaluation of noncalcified breast lesions. Radiology 266:89-95.

22. Michell M, Iqbal A, Wasan RK, et al (2012). A comparison of the accuracy of film-screen mammography, full-field digital mammography, and digital breast tomosynthesis. Clin Radiol 67:976-981.

23. Badr S, Laurent N, Régis C, et al (2013). Dual-energy contrast-enhanced digital mammography in routine clinical practice in 2013. Diagn Interv Imaging, Nov 12, 
24. Lobbes MB, Cleutjens JP, Lima Passos V, et al (2012). Density is in the eye of the beholder: Visual versus semi-automated assessment of breast density on standard mammograms. Insights Imaging 3:91-99. 



\section{6}

Contrast-enhanced spectral mammography in recalls from the dutch breast cancer screening program: validation of results in a large multireader, multicase study

Ulrich C. Lalji

Ivo P. L. Houben

Renate Prevos

Suzanne Gommers

Mireille van Goethem

Sigrid Vanwetswinkel

Ruud M. Pijnappel

Rachelle Steeman

Caroline Frotscher

Wai-Lan Mok,

Patricia J. Nelemans

Marjolein L. Smidt

Regina G. H. Beets-Tan

Joachim E. Wildberger

Marc B. I. Lobbes

Accepted march 2016 European Radiology: In press 


\section{Abstract}

Objective:- Contrast-enhanced spectral mammography (CESM) is a promising problem-solving tool in women referred from the breast cancer screening program. We aimed to study the validity of preliminary results of CESM using a larger panel of radiologists with different levels of CESM experience.

Material and methods - All women referred from the Dutch breast cancer screening program were eligible for CESM. 199 consecutive cases were viewed by ten radiologists. Four had extensive CESM experience, three had no CESM experience but were experienced breast radiologists, and three were residents. All readers provided a BI-RADS score for the low-energy CESM images first, after which the score could be adjusted when viewing the entire CESM exam . BI-RADS 1-3 were considered benign and BI-RADS 4-5 malignant. With this cut off, we calculated sensitivity, specificity and area under the ROC curve.

Results -CESM increased diagnostic accuracy in all readers. The performance for all readers using CESM was: sensitivity $96.9 \%$ (+3.9\%), specificity $69.7 \%$ (+33.8\%) and area under the ROC curve $0.833(+0.188)$.

Conclusion - CESM is superior to conventional mammography with excellent problem-solving capabilities in women referred from the breast cancer screening program. Previous results were confirmed even in a larger panel of readers with varying CESM experience. 


\section{Introduction}

In breast imaging, mammography plays a pivotal role in breast cancer detection and evaluation. Although the diagnostic accuracy of conventional mammography has improved significantly during the last decade due to the introduction of fullfield digital mammography (FFDM), its accuracy remains dependent on the density of the fibroglandular tissue [1].

Several new mammographic techniques have been introduced to improve FFDM's diagnostic accuracy, the most recent one being contrast-enhanced spectral mammography (CESM). Previous studies have shown that CESM is superior to FFDM for breast cancer detection, even equaling the performance of breast MRI [2-4]. It was also demonstrated that CESM was clinically feasible even in a study population with a low disease prevalence, i.e. recalls from a breast cancer screening program [5]. Although all diagnostic performance parameters in this study improved when using CESM, the most important changes were observed regarding specificity (increasing from $42 \%$ to $87.7 \%$ ) and positive predictive value (PPV, increasing from $39.7 \%$ to $76.2 \%$ ). These results showed that in this population CESM has great potential as a problem-solving tool. However, they were based on an interim analysis of the institution's first 113 patients and readings were performed by only two radiologists that were experienced in reading CESM exams.

Reproducibility of initial findings is an important step in the evaluation of every new diagnostic technique. Therefore, we aimed to compare the diagnostic performance of FFDM and CESM using a larger panel of radiologists with different levels of CESM experience[5].

\section{Materials and methods}

\section{Patient selection}

For this retrospective study, the requirement for obtaining informed consent was waived by the local ethics committee. All women recalled from the breast cancer screening program who were referred to our institution for assessment in the period November 2012 until October 2013 were eligible to undergo CESM. Women with a known allergy for iodinated contrast agents and those who had an increased risk for developing contrast induced nephropathy were excluded. The latter was established using the ESUR guidelines on Contrast Media as stated by the European Society of Urogenital Radiology (ESUR) [6].

\section{Imaging protocol and analysis}

The principle of the CESM technique was described elsewhere [7]. In short, a lowenergy (LE) and a high-energy image (HE) is obtained of both breasts in the standard mediolateral oblique (MLO) and craniocaudal (CC) views. The LE images pro- 
vide maximal soft tissue contrast and are similar to a conventional mammogram [8-10]. The HE image is not of diagnostic quality and is used for post-processing purposes only. Both images are used to create a recombined image which shows enhancement of lesions [7].

All CESM exams were performed on a single CESM unit (Senographe* Essential with Senobright* upgrade, GE Healthcare, Chalfont St Giles, United Kingdom) using a non-ionic monomeric low-osmolar contrast agent at a dose of $1.5 \mathrm{~mL} / \mathrm{kg}$ body weight (iopromide, Ultravist ${ }^{\circledR}$ 300, Bayer Healthcare, Berlin, Germany). Iodinated contrast was intravenously administered with a flow rate of $3 \mathrm{~mL} / \mathrm{s}$ two minutes prior to image acquisition. Both breasts were imaged in MLO and CC views with additional views to be requested by the radiologist if deemed necessary at the time of the exam. Patients were monitored for a minimal period of 30 minutes afterwards to rule out late adverse contrast reactions.

The panel of readers consisted of seven dedicated breast radiologists and three residents. Of the dedicated breast radiologists, four had two years' experience with CESM (the experienced CESM users). Their range of experience with conventional mammography was 2-6 years. The remaining three dedicated breast radiologists had between 3-25 years of experience with mammography but no previous experience whatsoever regarding CESM. The residents had limited CESM and mammography experience (eight weeks full time as part of their residency) and were trained in that period by the experienced CESM radiologists.

The panel of readers were allowed to learn the reason of referral from the screening program (similar to everyday clinical practice) and started by evaluating the LE image first.An initial BI-RADS score of 1 to 5 had to be provided before evaluating the entire CESM exam, including both the LE and recombined images, during the same reading session. The reader was then allowed to upgrade or downgrade their BI-RADS score if deemed necessary. All radiologists were blinded for each other's scores, previous or follow up examinations and final diagnosis. Readers were divided into 3 subgroups: experienced CESM readers, nonexperienced CESM readers and resident readers.

\section{Standard reference procedures used to assess true disease status}

To assess the true disease status of a recalled patient, one of the strategies below was followed [11].

In case of suspicious calcifications or masses a biopsy was performed under ultrasound guidance or stereotactic guidance with histology serving as gold standard. In all cases of cysts, a targeted ultrasound examination was performed in combination with aspiration of the cyst to prove its non-solid nature. In cases where superposition of normal fibroglandular tissue was suspected, at least one additional view of the breast containing the suspicious lesion followed by targeted ultrasound was performed. If no abnormality was found on additional imaging, women were discharged according to the NHS Breast Screening Program 
(NHSBSP) Clinical Guidelines for Breast Cancer Screening Assessment and our national guidelines[12,13].

\section{Statistical analysis}

BI-RADS scores 1-3 were considered benign and BI-RADS 4-5 malignant. Using these cut-off values sensitivity, specificity, positive predictive value (PPV) and negative predictive value (NPV) of the readers were calculated. Interpretation of images from the same set of patients by multiple readers are likely to be correlated. Moreover, because FFDM and CESM were performed in the same study population, the results of both tests are also correlated. Ignoring correlation can lead to misleadingly small estimates of the standard error and consequently too small 95\% confidence intervals which are too small. To adjust for the correlated data structure, variance of the sensitivity and specificity for all readers and for subgroups of readers (experienced in CESM reading, non-experienced in CESM reading and residents) were adjusted with the variance inflation factor (VIF). The variance of the difference in sensitivity and specificity between FFDM and CESM was estimated based on these adjusted variances and the covariance between FFDM and CESM results. Adjusted 95\% confidence intervals (CI) were derived using an excel spreadsheet provided by Genders et al. [14].

Receiver operating characteristic (ROC) curves were constructed for both imaging modalities and areas under the curve (AUC) with corresponding 95\% CI were calculated using bootstrap analyses (2.000 repetitions). The DeLong test was used for paired comparison of the AUC of FFDM and CESM[15]. Fleiss' generalized kappa coefficient was used to determine the interrater reliability for the image analysis of both FFDM and CESM.

In a separate analysis, false negative and false positive findings were further evaluated. All false negatives were analyzed to identify potential CESM pitfalls. For false positives findings, cases were included for this sub analysis if five or more readers (i.e. more than half of the reading panel) scored this case as being false positive on CESM. All statistical analyses were performed using SPSS (IBM Corp. Released 2011. IBM SPSS Statistics for Windows, Version 20.0. Armonk, NY: IBM Corp.) and the , pROC package in R (Version: 1.7.2 released on April 6th, 2014)[15]. P-values $<0.05$ were considered statistically significant.

\section{Results}

A total of 199 consecutive patients who were referred to our institution from the breast cancer screening program underwent CESM as part of their workup (mean age 58.4 years, range $49-75$ years). Most recalls were caused by masses $(76.4 \%)$, followed by calcifications (15.1\%), asymmetry (5.0\%) and architectural distortions 


\section{4 | Chapter 6}

(3.5\%). At final diagnosis, $29.6 \%$ of the cases proved to be malignant. Detailed patient characteristics were summarized in Table 1.

Table 2 presents detailed information of sensitivity and specificity for all readers. For the entire reading panel, diagnostic performance parameters improved when using CESM. Mean sensitivity increased from $93.0 \%$ to $96.9 \%$ and mean specificity from $35.9 \%$ to $69.7 \%$. Mean PPV and NPV increased from $38.7 \%$ and $92.6 \%$ to $58.2 \%$ and $98.2 \%$, respectively. The ROC curves showed an improvement in diagnostic performance for all readers when using CESM (Figure 1). For all readers combined, the AUC value increased from 0.645 to 0.833 ( $p<0.0001)$. Detailed results for the comparison of CESM and FFDM are presented in Table 3. Sensitivity increased for all reader panels but was only significantly increased for resident readers $(p=0.011)$ and for all readers together $(p=0.0002)$. Looking at the difference in sensitivity and specificity for CESM and FFDM both increased for all readers using CESM. For all subgroups of reader panels specificity was significantly increased. The interrater variability was considered to be excellent with a kappavalue of 0.89 . 
Table 1:Patient characteristics: age, final diagnosis for malignant and benign lesions and subtypes of invasive cancers given. Abbreviations: ER: oestrogen receptor, PR: progesterone receptor, HER2/neu: Human Epidermal growth factor Receptor 2.

\begin{tabular}{ll}
\hline Age & Years \\
\hline Mean & 58.4 \\
Standard deviation & 8.1 \\
Range & $49-75$
\end{tabular}

Final Diagnosis Percentage (n=199)

Malignant

Invasive ductal carcinoma

Invasive lobular carcinoma

Ductal carcinoma in situ

2.5

Invasive mucinous carcinoma

Invasive micropapillary carcinoma

Benign

Fibroadenoma

Simple cyst

Reactive changes / benign

Apocrine changes / metaplasia

Papilloma

Superposition

Cylindrical cell changes

Old hematoma

0.5

Inflammation

Intramammary lymph node

2.0

Sclerosing adenosis

Atypical lobular hyperplasia

0.5

Ductectasia

Fibrosis

Ductal hyperplasia

Lobular carcinoma in situ

0.5

Flat epithelial atypia

0.5

Total

100.0

\begin{tabular}{ll}
\hline Invasive breast cancer subtypes & Percentage of all invasive cancers \\
\hline ER positive & 92.3 \\
PR positive & 80.8 \\
HER2 $/$ neu positive & 12.2 \\
Grade 1 & 27.3 \\
Grade 2 & 47.3 \\
Grade 3 & 25.5 \\
\hline
\end{tabular}


Ten cases (5\%) with false negative CESM findings were observed. An overview of the final diagnosis, tumor characteristics and the number of readers that scored the individual case as false negative is presented in Table 4 . Three readers had no false negative scores with CESM. All other readers had at least one false-negative finding on CESM (median 2 cases, range 1-4).

Table 2. Diagnostic performance of FFDM and CESM for all ten readers. Diagnostic performance parameters were presented as percentages with 95\% confidence intervals in parentheses.

\begin{tabular}{|c|c|c|c|}
\hline Reader & Exam & Sensitivity & Specificity \\
\hline \multirow[t]{2}{*}{ Experienced CESM reader 1} & FFDM & $86.4 \%(75.0-93.9 \%)$ & $67.1 \%(58.7-74.8 \%)$ \\
\hline & CESM & $93.2 \%(83.5-98.1 \%)$ & $86.4 \%(79.6-91.6 \%)$ \\
\hline \multirow[t]{2}{*}{ Experienced CESM reader 2} & FFDM & $96.6 \%(88.2-99.4 \%)$ & $26.4 \%(19.3-34.5 \%)$ \\
\hline & CESM & $98.3 \%(90.8-99.7 \%)$ & $70.7 \%(62.4-78.1 \%)$ \\
\hline \multirow[t]{2}{*}{ Experienced CESM reader 3} & FFDM & $94.9 \%(85.8-98.9 \%)$ & $49.3 \%(40.7-57.8 \%)$ \\
\hline & CESM & $100.0 \%(93.8-100.0 \%)$ & $75.7 \%(67.7-82.6 \%)$ \\
\hline \multirow[t]{4}{*}{ Experienced CESM reader 4} & FFDM & $98.3 \%(90.8-99.7 \%)$ & $15.0 \%(9.5-22.0 \%)$ \\
\hline & CESM & $100.0 \%(93.8 \%-100.0 \%)$ & $75.7 \%(67.7-82.5 \%)$ \\
\hline & Mean FFDM & $94.1 \%(89.6-98.5 \%)$ & $39.5 \%(19.7-59.2 \%)$ \\
\hline & Mean CESM & $97.6 \%(95.1-100 \%)$ & $77.1 \%(71.5-82.7 \%)$ \\
\hline \multirow[t]{2}{*}{ Non-experienced CESM reader 1} & FFDM & $98.3 \%(90.8-99.7 \%)$ & $37.8 \%(29.8-56.4 \%)$ \\
\hline & CESM & $100.0 \%(93.8-100.0 \%)$ & $67.1 \%(58.7-74.8 \%)$ \\
\hline \multirow[t]{2}{*}{ Non-experienced CESM reader 2} & FFDM & $96.6 \%(88.2-99.5 \%)$ & $21.4 \%(14.9-29.1 \%)$ \\
\hline & CESM & $94.9 \%(85.8-98.8 \%)$ & $64.3 \%(55.7-72.2 \%)$ \\
\hline \multirow[t]{4}{*}{ Non-experienced CESM reader 3} & FFDM & $89.9 \%(79.1-96.1 \%)$ & $40.7 \%(32.5-49.3 \%)$ \\
\hline & CESM & $93.2 \%(83.5-98.1 \%)$ & $72.8 \%(64.7-80.0 \%)$ \\
\hline & Mean FFDM & $94.9 \%(90.8-99.0 \%)$ & $33.3 \%(23.7-42.9 \%)$ \\
\hline & Mean CESM & $95.9 \%(92.9-98.9 \%)$ & $68.0 \%(64.1-72.1 \%)$ \\
\hline \multirow{2}{*}{$\begin{array}{l}\text { Resident } \\
\text { Reader } 1\end{array}$} & FFDM & $89.8 \%(79.1-96.1 \%)$ & $32.8 \%(25.1-41.3 \%)$ \\
\hline & CESM & $96.6 \%(88.2-99.4 \%)$ & $58.5 \%$ (49.9-66.8\% \\
\hline \multirow{2}{*}{$\begin{array}{l}\text { Resident } \\
\text { Reader } 2\end{array}$} & FFDM & $93.2 \%(83.5-98.0 \%)$ & $36.4 \%(36.4-44.9 \%)$ \\
\hline & CESM & $96.6 \%(88.2-99.4 \%)$ & $64.2 \%(55.7-72.2 \%)$ \\
\hline \multirow{4}{*}{$\begin{array}{l}\text { Resident } \\
\text { Reader } 3\end{array}$} & FFDM & $86.4 \%(75.0-93.9 \%)$ & $32.1 \%(24.5-40.5 \%)$ \\
\hline & CESM & $96.6 \%(88.2-99.5 \%)$ & $61.4 \%(52.8-69.5 \%)$ \\
\hline & Mean FFDM & $89.8 \%(79.2-96 \%)$ & $33.7 \%(28.6-42.2 \%)$ \\
\hline & Mean CESM & $96.6 \%(95.4-98.2 \%)$ & $61.3 \%(52.8-69.5 \%)$ \\
\hline \multirow[t]{2}{*}{ All readers mean } & FFDM & $93.0 \%(90.3-95.8 \%)$ & $35.9 \%(27.3-44.5 \%)$ \\
\hline & CESM & $96.9 \%(93.2-100.0 \%)$ & $69.7 \%(64.8-74.6 \%)$ \\
\hline
\end{tabular}


False positive readings by 5 or more readers

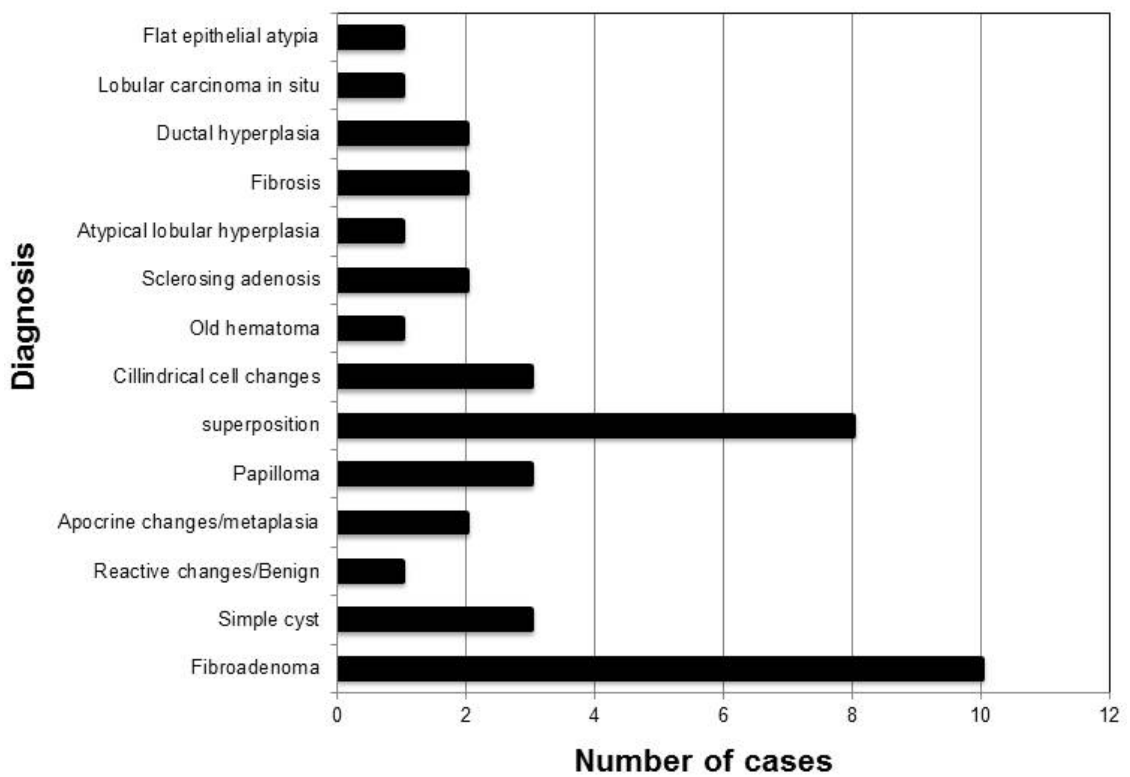

Figure 2: Overview of number of cases and diagnosis of false-positive findings. These cases were scored as false positive cases by five or more readers.

The mean number of false positive cases was 42 (21.1\%, range 19-58), with an average for the experienced CESM readers of 31 cases $(15.6 \%)$. The experienced FFDM readers showed an average of 37 false positive cases (18.6\%), whereas the residents showed 54 false positive findings (27.1\%). A total of 40 cases $(20.1 \%)$ were scored as false positive by five or more readers using CESM. In this sub group, the most common causes were fibroadenomas $(n=10)$, followed by superposition densities $(n=8)$, and cysts $(n=3)$. A detailed summary of this sub analysis is presented in Figure 2.

Table 3: Difference $(\Delta)$ in sensitivity and specificity of CESM and FFDM with 95\% confidence Intervals (CI) in parenthesis. P-Values (p) $<0.05$ are considered significant.

\begin{tabular}{|c|c|c|c|c|}
\hline & $\begin{array}{l}\Delta \text { Sensitivity } \\
\text { CESM-FFDM }(95 \% \mathrm{CI})\end{array}$ & $\begin{array}{l}\text { p- value } \\
(<0.05 \text { significant })\end{array}$ & $\begin{array}{l}\Delta \text { Specificity } \\
\text { CESM-FFDM }(95 \% \mathrm{CI})\end{array}$ & $\begin{array}{l}p \text {-value } \\
(<0.05 \text { significant })\end{array}$ \\
\hline $\begin{array}{l}\text { Experienced CESM } \\
\text { readers }\end{array}$ & $0.035(-0.009-0.079)$ & $\mathrm{p}=0.114$ & $0.376(0.193-0.559)$ & $p=0.000056$ \\
\hline $\begin{array}{l}\text { Non-experienced } \\
\text { CESM readers }\end{array}$ & $0.010(0.036-0.056)$ & $p=0.667$ & $0.348(0.254-0.442)$ & $\mathrm{p}<0.00001$ \\
\hline $\begin{array}{l}\text { Resident } \\
\text { Readers }\end{array}$ & $0.068(0.016-0.120)$ & $p=0.011$ & $0.276(0.223-0.329)$ & $\mathrm{p}<0.00001$ \\
\hline All readers & $0.038(0.018-0.058)$ & $\mathrm{p}=0.0002$ & $0.338(0.267-0.409)$ & $\mathrm{p}<0.00001$ \\
\hline
\end{tabular}


ALL reviewers

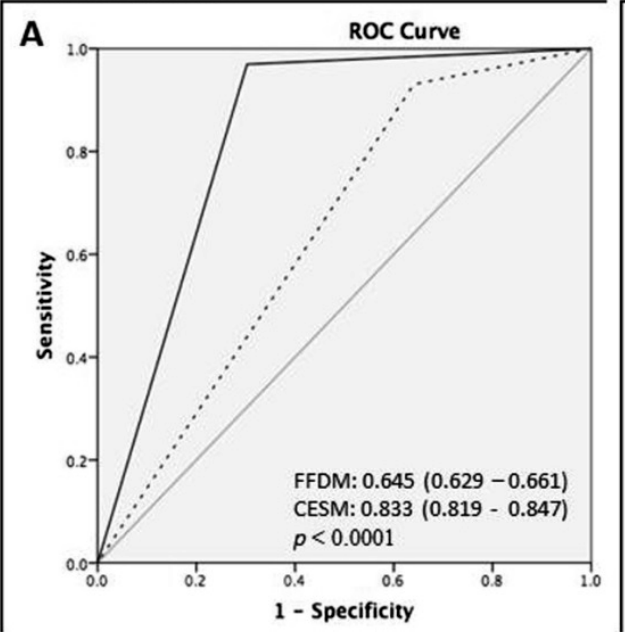

Non - Experienced reviewers

C

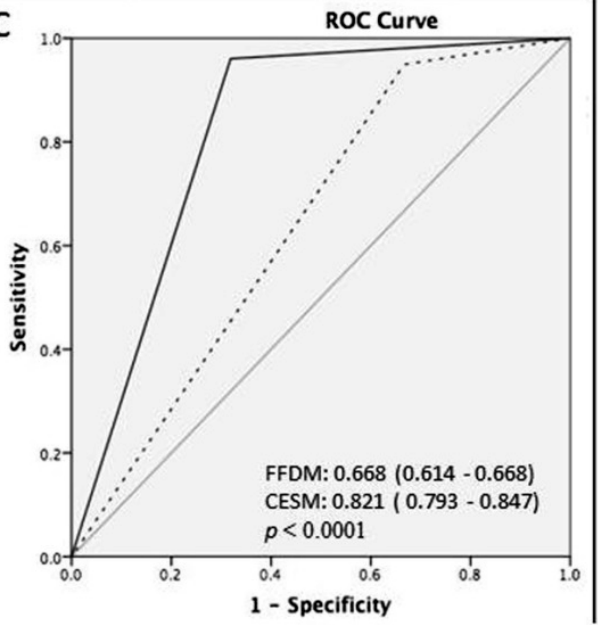

Experienced reviewers

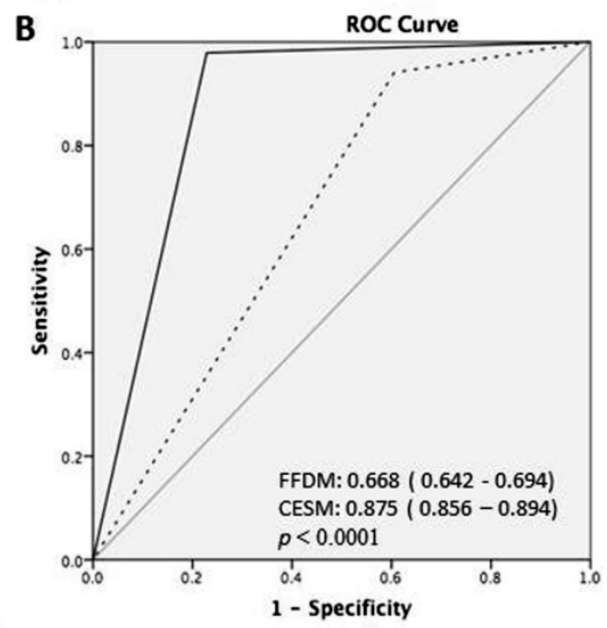

Resident reviewers

D
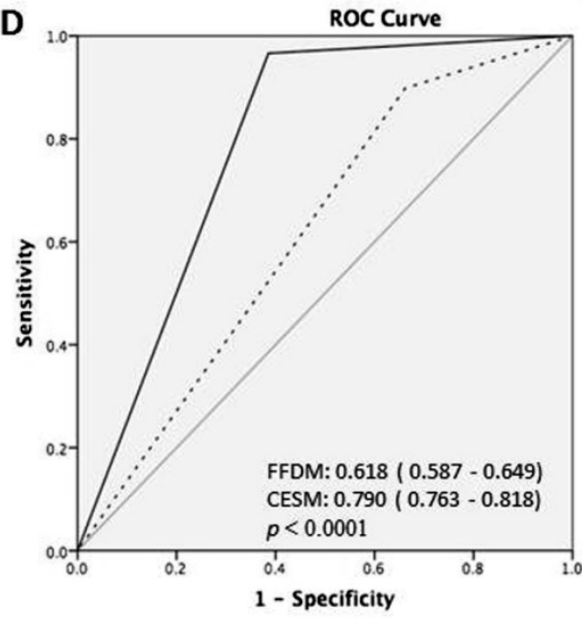

\section{-- FFDM \\ - CESM \\ - Reference line}

Figure 1. Average ROC curves for all readers (A), experienced CESM readers (B), experienced FFDM readers (C) and resident readers (D). AUC values for FFDM and CESM given with confidence intervals in parenthesis. Differences in AUC between FFDM and CESM were significantly increased for all subgroup of reader panels, $\mathrm{p}$-values given per subgroup of reader panel. 
Table 4. Diagnosis of false negative cases and the number of readers that scored them as false negative. Experience level is indicated of the number of readers that missed the lesion on CESM. In addition lesion characteristics such as diameter (given in millimetres), histologic grade, DCIS grade and hormonal receptor status (ER, PR, HER2NEU) are given. Hormone receptor status in case of pure DCIS is not evaluated and therefore not available (n/a) for these cases.

\begin{tabular}{|c|c|c|c|c|c|c|c|c|c|}
\hline \multirow{2}{*}{$\begin{array}{l}\text { Final } \\
\text { diagnosis } \\
\text { Histology }\end{array}$} & \multicolumn{4}{|c|}{ Number of readers scoring false negative } & \multicolumn{5}{|c|}{ Tumour Characteristics } \\
\hline & $\begin{array}{l}\text { Experienced } \\
\text { CESM }\end{array}$ & $\begin{array}{c}\text { Non- } \\
\text { experienced } \\
\text { CESM }\end{array}$ & Resident & Total & $\begin{array}{l}\text { diameter } \\
\text { in } \mathrm{mm}\end{array}$ & Grade & ER & PR & HER2/neu \\
\hline $\begin{array}{l}\text { Invasive } \\
\text { ductal } \\
\text { carcinoma }\end{array}$ & 1 & 2 & 2 & 5 & 14 & 2 & + & + & - \\
\hline $\begin{array}{l}\text { Invasive } \\
\text { ductal } \\
\text { carcinoma }\end{array}$ & 1 & 1 & 1 & 3 & 22 & 2 & + & + & + \\
\hline $\begin{array}{l}\text { Invasive } \\
\text { mucinous } \\
\text { carcinoma }\end{array}$ & - & 2 & - & 2 & 18 & 1 & + & + & - \\
\hline $\begin{array}{l}\text { Invasive } \\
\text { ductal } \\
\text { carcinoma }\end{array}$ & - & 1 & - & 1 & 20 & 2 & + & - & - \\
\hline $\begin{array}{l}\text { Invasive } \\
\text { ductal } \\
\text { carcinoma }\end{array}$ & 1 & - & - & 1 & 7 & 1 & + & + & - \\
\hline $\begin{array}{l}\text { Invasive } \\
\text { ductal } \\
\text { carcinoma }\end{array}$ & 1 & - & - & 1 & 4 & 1 & + & - & - \\
\hline $\begin{array}{l}\text { Invasive } \\
\text { ductal } \\
\text { carcinoma }\end{array}$ & - & - & 1 & 1 & 16 & 2 & + & + & - \\
\hline $\begin{array}{l}\text { Ductal } \\
\text { carcinoma in } \\
\text { situ }\end{array}$ & - & 1 & - & 1 & 10 & $2-3$ & $\mathrm{n} / \mathrm{a}$ & $\mathrm{n} / \mathrm{a}$ & $\mathrm{n} / \mathrm{a}$ \\
\hline $\begin{array}{l}\text { Invasive } \\
\text { lobular } \\
\text { carcinoma }\end{array}$ & - & - & 1 & 1 & 5 & 2 & + & + & - \\
\hline $\begin{array}{l}\text { Ductal } \\
\text { carcinoma in } \\
\text { situ }\end{array}$ & - & - & 1 & 1 & 26 & 3 & $\mathrm{n} / \mathrm{a}$ & $\mathrm{n} / \mathrm{a}$ & $\mathrm{n} / \mathrm{a}$ \\
\hline
\end{tabular}

\section{Discussion}

CESM is a promising new breast imaging modality. In CESM, an iodine-based contrast agent is intravenously administered, after which dual-energy mammography is performed. As a result, the radiologist can view a low-energy image (which is similar to a conventional FFDM) and a recombined image, showing areas of enhancement $[7,16]$. In a previous study it was shown that CESM is an excellent 
problem solving tool for women recalled from the breast cancer screening program[5]. However, these results were based on an interim analysis of the institution's first 113 cases read by only two radiologists experienced in CESM. Our current study shows that these results were reproducible, even in a large number of cases read by a panel of ten different radiologists with varying experience in reading CESM exams. Mean sensitivity increased from $93.0 \%$ to $96.9 \%$ and mean specificity from $35.9 \%$ to $69.7 \%$. Mean PPV and NPV increased from $38.7 \%$ and $92.6 \%$ to $58.2 \%$ and $98.2 \%$, respectively.

Several publications have studied the diagnostic performance of CESM (Table 5) $[2-5,8,17-22]$. In these studies, the mean sensitivity of CESM varied from 77.8 $100.0 \%$, whereas mean specificity (if available) varied from 41-87.7\%. In some studies specificity could not be calculated since all included subjects were diagnosed with breast cancer $[3,8,19]$. One study did not provide specificity but accuracy instead [22].The disease prevalence in the other studies (except the study by Lobbes et al.) were higher than our population (range 36-100\%). The reported prevalence of $36 \%$ concerned a study population where all subjects included had microcalcifications without an associated mass[21]. However, breast cancer prevalence in clinical practice is low. It is interesting to study the diagnostic performance of CESM in populations with low breast cancer prevalence since it should not result in a large number of false-positive findings.

Table 5. Studies comparing CESM and Mammography: number of patients included, sensitivity and specificity given for CESM. also disease prevalence based on number of lesions analysed in the included population is given, calculated from data given in study.

\begin{tabular}{lcclc}
\hline Study & $\begin{array}{l}\text { Number of patients } \\
(\mathrm{n}=)\end{array}$ & Sensitivity (\%) & Specificity (\%) & $\begin{array}{l}\text { Disease } \\
\text { prevalence (\%) }\end{array}$ \\
\hline Lewin et al.(2003) & 26 & 100 & 86.7 & 50 \\
Dromain et al. (2011) & 144 & 93 & 63 & 56.3 \\
Dromain et al. (2012) & 110 & 91.9 & 46 & 56.7 \\
Fallenberg et al. (2013) & 80 & 100 & - & 100 \\
Jochelson et al. (2013) & 52 & 96 & - & 100 \\
Fallenberg et al. (2014)1 & 118 & $94.7^{*} / 95^{* *}$ & - & 100 \\
Lobbes et al. (2014) & 113 & 100 & 87.7 & 28 \\
Luczyńska et al. (2014) & 152 & 100 & 41 & 76 \\
Cheung et al (2014) & 89 & 92.7 & 67.9 & 72 \\
Luczyńska et al. (2015) & 118 & 100 & Not provided & 68.6 \\
Cheung et al (2015)2 & 52 & 90.9 & 83.78 & 37.7 \\
\hline
\end{tabular}

Annotations: 1sensitivity for CESM alone(*) and for CESM in combination with mammography(**). 2 Patients with microcalcifications only.

For this reason, Lobbes et al. studied CESM's diagnostic performance in women recalled from breast cancer screening, who had a breast cancer prevalence of 28.3\%[5]. They found that (when compared to FFDM) sensitivity increased from $96.9 \%$ to $100.0 \%$ and NPV increased from $97.1 \%$ to $100.0 \%$. Interestingly, the 
largest improvements were observed for specificity and PPV, increasing from $42.0 \%$ and $39.7 \%$ to $87.7 \%$ and $76.2 \%$, respectively. It was concluded that CESM was an excellent problem solving imaging modality for recalls from the breast cancer screening program, able to detect breast cancer accurately, while establishing false-positive recalls confidently.

An important limitation of the study by Lobbes et al. was that two readers experienced with CESM read the cases. However, in order to become clinically implemented, the reproducibility of test results of every new diagnostic imaging modality should be validated, preferably in larger study populations using multiple readers. Therefore, we used a panel of ten different readers with different experience in FFDM and CESM to evaluate 199 consecutive CESM exams of women recalled from the breast cancer screening program. Our current results confirmed prior observations, with an increase of all diagnostic performance parameters when using CESM, especially with respect to specificity and PPV. These improvements were observed for all readers, independent of their level of CESM experience. Current results are in line with another previously published study with relatively lower disease prevalence. Luczyńska et al. studied 157 breast lesions (breast cancer prevalence 38.3\%) using both FFDM and CESM [4]. Sensitivity of CESM was $100 \%$, with a PPV and NPV of $77 \%$ and $100 \%$, respectively with an AUC of 0.86 . However, in their study only a single reader was used to view the exams.

CESM has potential pitfalls, resulting in both false negative or false positive findings. In a study by Thibault et al. six false negative findings were observed: two invasive ductal carcinomas outside the field of view and four invasive lobular cancers [23]. Fallenberg et al. (using three readers) found that when CESM was solely used one cancer was missed by all readers, four cancers were missed by two, and three by one reader [8]. In our study a total of 10 false negative cases were observed, scored incorrectly by one or more readers.

The cancers that were overlooked by more than one reader were analyzed case-by-case. These were: one invasive grade 2 ductal carcinoma missed by five readers, one invasive grade 2 ductal carcinoma missed by three readers, and one grade 1 invasive mucinous carcinoma missed by two readers. These cases are illustrated in Figure 3. Two cases consisted of a focal asymmetry with ill-defined margins, only partly visible on the MLO view only. These lesions showed only subtle or no enhancement on the recombined images (Figure 3A and 3B). Among the readers that missed these lesions were experienced CESM users as well as none experienced CESM users and residents. Future developments such as computer aided detection systems for CESM or contrast-enhanced digital breast tomosynthesis, could potentially reduce the risk of missing these types of lesions.

The third case (missed by two readers) consisted of an ill-defined mass visible on both CC and MLO views with a central coarse calcification without any enhancement on the recombined images. Despite the lack of enhancement it does not represent a typical 'eclipse' sign which is the CESM appearance of a cyst, consisting of a dark 'void' on the recombined images combined with a subtle rim enhancement, resembling a solar eclipse [5]. This atypical appearance of the eclipse sign 
together with its irregular margin warranted additional targeted ultrasound. Final pathology showed a grade 1 mucinous carcinoma (Figure 3C). Mucinous carcinomas can be a CESM pitfall due to lack of enhancement. This case demonstrates that readers should not only focus on the recombined images. They are an adjunct to the mammographic images, not a replacement.
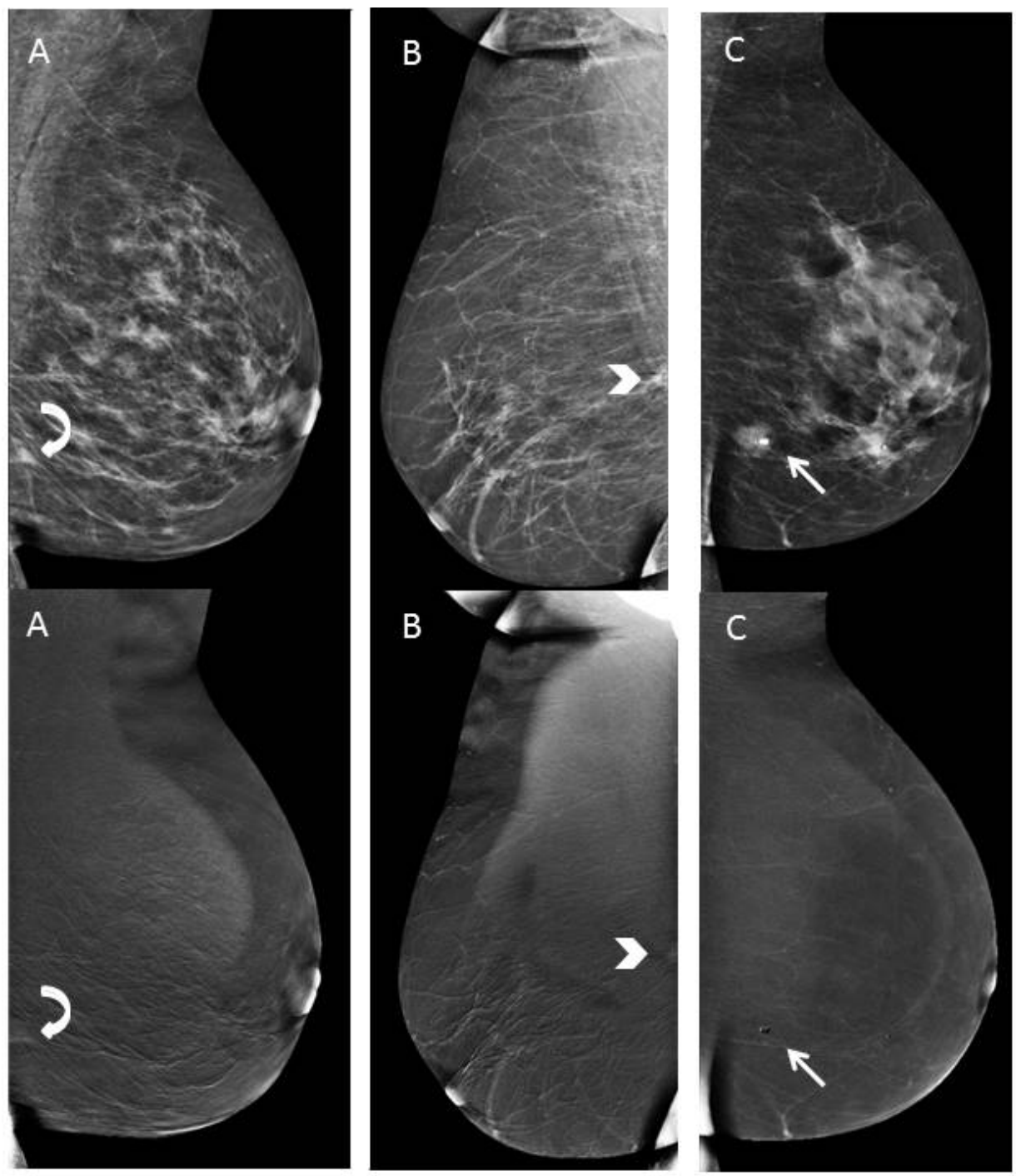

Figure 3. Example of false negative cases. Low-energy images at the top with correspnding recombined images underneath. A: infiltrating grade 2 ductal carcinoma with grade 3 ductal carcinoma in situ (curved arrow). B: invasive grade 2 ductal carcinoma (arrow head). C: grade 1 mucinous carcinoma (straight arrow).

CESM also generates false positive findings. In the study by Badr et al. enhancement was observed in $33 \%$ of 27 benign lesions [24]. Jochelson et al. detected two false positive results in 52 patients (4\%) using CESM [3]. Lobbes et al. detected five 
false positive findings in a population of 113 women [[5]]. Luczyńska et al. found 35 (20\%) false positive lesions with CESM compared to 50 (29\%) with conventional mammography [4]. Similar to our observations, most of these lesions were caused by fibroadenomas $(n=26)$ or some other benign solid breast lesion. Although these findings resulted in tissue sampling which could have been avoided, its prevalence is low and do not outweigh the improved cancer detection rates caused by CESM when compared to FFDM.

This study showed that CESM remains an excellent problem-solving tool for patients recalled from breast cancer screening, even when radiologists less experienced in CESM are reviewing the images. This implies that reading CESM exams hardly has any learning curve. Introduction into everyday clinical practice is safe and feasible [5]. Using CESM in recalled patients increases specificity and PPV, thus providing the radiologist with a confident final diagnosis in cases of false positive recalls. For example, if recalled patients have a negative CESM exam the high NPV rules out the presence of breast cancer, preventing people from undergoing additional exams (such as breast MRI) or follow-up exams. Nevertheless, prospective randomized controlled trials are necessary comparing the standard work-up using conventional breast imaging with CESM-based work-up in order to accept CESM as a primary imaging tool in the work-up of recalled patients.

\section{Study limitations}

Our study had some limitations. Earlier results were previously published and consisted of 113 cases read by two experienced CESM viewers [5]. In the current study, these two readers were again participating in the scoring of the exams, thus re-scoring these exams. However, the data used was anonymized and the time period between the two scoring rounds was more than one year, minimizing the chances of introducing recall bias in these 113 cases. The remaining 86 cases were also new to these two readers.

To prove that no recall bias was introduced, we performed additional analyses. In the previous publication, the AUC of ROC curve was 0.779 for mammography, increasing to 0.976 using CESM [5]. These were the results of 113 cases. After more than one year, two readers reviewed these 113 cases again as part of the current study, achieving a diagnostic performance as expressed by the AUC value of 0.831 for mammography and 0.971 using CESM. The AUC value of the final 86 (completely new) cases was 0.881 for mammography and 0.977 for CESM. This shows that no recall bias is introduced during the re-reading of the first 113 cases by these two readers. For the remaining eight readers all 199 cases are completely new.

For reasons of comparison, we decided to include the complete data of the two experienced CESM reviewers to provide an overview of the performance of each reader for the entire case collection. However, in order to further assess the reproducibility of these results, it would be valuable to conduct a study consisting of an 
entirely different population, preferably in different institutes, using units of different vendors, that are now becoming commercially available.

Second, there was no follow-up of cases with superposition of fibroglandular tissue as final diagnosis. However, our current imaging strategy of these cases complies with the NHSBSP's Clinical Guidelines for Breast Cancer Screening Assessment [12]. This strategy is safe, with the chances of overlooking breast cancer being minimal, as was additionally proven by an institutional quality control covering almost 600 recalls from screening (personal communication).

Third, all cases were recalls from the national breast cancer screening program. This introduced some selection bias since all patients were pre-selected by two screening radiologists. In addition, readers were not blinded for the reason of referral. However, the latter two limitations reflect everyday clinical practice of the work-up of recalled women.

Finally, the additional value of (targeted) ultrasound next to FFDM was not taken into account since we wished to focus on the additional value of adding contrast and the recombined images to conventional mammography. Indeed, additional ultrasound could also clarify some findings that proved to be benign (such as a cyst). Since Dromain et al. showed that CESM is also superior to mammography and ultrasound combined [2], it would still be recommendable to use CESM as a primary imaging tool for recalled patients.

\section{Conclusion}

The diagnostic performance of CESM is superior to FFDM in women recalled from the breast cancer screening program, confirming previously published results. Even when used by less experienced CESM readers, CESM increases all diagnostic accuracy parameters, especially specificity and positive predictive value. 


\section{References}

1. Carney PA, Miglioretti DL, Yankaskas BC, et al. (2003) Individual and combined effects of age, breast density, and hormone replacement therapy use on the accuracy of screening mammography. Ann Intern Med 138:168-75.

2. Dromain C, Thibault F, Muller S, et al. (2011) Dual-energy contrast-enhanced digital mammography: initial clinical results. Eur Radiol 21:565-74. doi: 10.1007/s00330-010-1944-y

3. Jochelson MS, Dershaw DD, Sung JS, et al. (2013) Bilateral contrast-enhanced dual-energy digital mammography: feasibility and comparison with conventional digital mammography and MR imaging in women with known breast carcinoma. Radiology 266:743-51. doi: 10.1148/radiol.12121084

4. Luczyńska E, Heinze-Paluchowska S, Dyczek S, et al. (2014) Contrast-enhanced spectral mammography: comparison with conventional mammography and histopathology in 152 women. Korean J Radiol 15:689-96. doi: 10.3348/kjr.2014.15.6.689

5. Lobbes MBI, Lalji U, Houwers J, et al. (2014) Contrast-enhanced spectral mammography in patients referred from the breast cancer screening programme. Eur Radiol 24:1668-76. doi: 10.1007/ s00330-014-3154-5

6. Stacul F, van der Molen AJ, Reimer P, et al. (2011) Contrast induced nephropathy: updated ESUR Contrast Media Safety Committee guidelines. Eur Radiol 21:2527-41. doi: 10.1007/s00330-0112225-0

7. Lobbes MBI, Smidt ML, Houwers J, et al. (2013) Contrast enhanced mammography: techniques, current results, and potential indications. Clin Radiol 68:935-44. doi: 10.1016/j.crad.2013.04.009

8. Fallenberg EM, Dromain C, Diekmann F, et al. (2014) Contrast-enhanced spectral mammography: Does mammography provide additional clinical benefits or can some radiation exposure be avoided? Breast Cancer Res Treat 146:371-81. doi: 10.1007/s10549-014-3023-6

9. Francescone MA, Jochelson MS, Dershaw DD, et al. (2014) Low energy mammogram obtained in contrast-enhanced digital mammography (CEDM) is comparable to routine full-field digital mammography (FFDM). Eur J Radiol 83:1350-5. doi: 10.1016/j.ejrad.2014.05.015

10. Lalji UC, Jeukens CRLPN, Houben I, et al. (2015) Evaluation of low-energy contrast-enhanced spectral mammography images by comparing them to full-field digital mammography using EUREF image quality criteria. Eur Radiol. doi: 10.1007/s00330-015-3695-2

11. Obuchowski NA (2003) Receiver operating characteristic curves and their use in radiology. Radiology 229:3-8.

12. Liston J, Wilson R (2010) NHSBSP clinical guidelines for breast cancer screening assessment, 3rd edn. NHS Cancer Screening Programmes, Sheffield. http://www.cancerscreening.nhs.uk/breastscreen/publications/nhsbsp49.pdf. Accessed 1 Mar 2015

13. Nationaal Borstkanker Overleg Nederland (NABON) National guideline breast cancer 2012, Amsterdam, NABON 2012 (2012) Mammacarcinoom.

14. Genders TSS, Spronk S, Stijnen T, et al. (2012) Methods for calculating sensitivity and specificity of clustered data: a tutorial. Radiology 265:910-6. doi: 10.1148/radiol.12120509

15. Robin X, Turck N, Hainard A, et al. (2011) pROC: an open-source package for R and S+ to analyze and compare ROC curves. BMC Bioinformatics 12:77. doi: 10.1186/1471-2105-12-77

16. Lalji U, Lobbes M (2014) Contrast-enhanced dual-energy mammography: a promising new imaging tool in breast cancer detection. Womens Health (Lond Engl) 10:289-98. doi: 10.2217/whe.14.18

17. Lewin JM, Isaacs PK, Vance V, Larke FJ (2003) Dual-energy contrast-enhanced digital subtraction mammography: feasibility. Radiology 229:261-8. doi: 10.1148/radiol.2291021276

18. Dromain C, Thibault F, Diekmann F, et al. (2012) Dual-energy contrast-enhanced digital mammography: initial clinical results of a multireader, multicase study. Breast Cancer Res 14:R94. doi: $10.1186 / \mathrm{bcr} 3210$

19. Fallenberg EM, Dromain C, Diekmann F, et al. (2013) Contrast-enhanced spectral mammography versus MRI: Initial results in the detection of breast cancer and assessment of tumour size. Eur Radiol. doi: 10.1007/s00330-013-3007-7

20. Cheung Y-C, Lin Y-C, Wan Y-L, et al. (2014) Diagnostic performance of dual-energy contrastenhanced subtracted mammography in dense breasts compared to mammography alone: interobserver blind-reading analysis. Eur Radiol. doi: 10.1007/s00330-014-3271-1 


\section{Chapter 6}

21. Cheung Y-C, Tsai H-P, Lo Y-F, et al. (2015) Clinical utility of dual-energy contrast-enhanced spectral mammography for breast microcalcifications without associated mass: a preliminary analysis. Eur Radiol. doi: 10.1007/s00330-015-3904-z

22. Łuczyńska E, Heinze-Paluchowska S, Hendrick E, et al. (2015) Comparison between breast MRI and contrast-enhanced spectral mammography. Med Sci Monit 21:1358-67. doi: 10.12659/MSM.893018

23. Thibault F, Balleyguier C, Tardivon A, Dromain C (2012) Contrast enhanced spectral mammography: better than MRI? Eur J Radiol 81 Suppl 1:S162-4. doi: 10.1016/S0720-048X(12)70068-2

24. Badr S, Laurent N, Régis C, et al. (2014) Dual-energy contrast-enhanced digital mammography in routine clinical practice in 2013. Diagn Interv Imaging 95:245-58. doi: 10.1016/j.diii.2013.10.002 


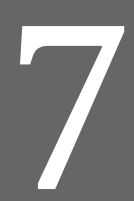

\section{The quality of tumour size assessment by contrast-enhanced spectral mammography and the benefit of additional breast MRI}

Marc B. I. Lobbes

Ulrich C. Lalji

Patty J. Nelemans

Ivo P. L. Houben

Marjolein L. Smidt

Esther Heuts

Bart de Vries

Joachim E. Wildberger

Regina G. H. Beets-Tan

Published in: Journal of Cancer 2015 


\section{Abstract}

Background: Contrast-enhanced spectral mammography (CESM) is a promising new breast imaging modality that is superior to conventional mammography for breast cancer detection. We aimed to evaluate correlation and agreement of tumour size measurements using CESM. As additional analysis, we evaluated whether measurements using an additional breast MRI exam would yield more accurate results.

Methods: Between January 1st 2013 and April 1st 2014, 87 consecutive breast cancer cases that underwent CESM were collected and data on maximum tumour size measurements were gathered. In 57 cases, tumour size measurements were also available for breast MRI. Histopathological results of the surgical specimen served as gold standard in all cases.

Results: The Pearson's correlation coefficients (PCC) of CESM versus histopathology and breast MRI versus histopathology were all $>0.9$, $p<0.0001$. For the agreement between measurements, the mean difference between CESM and histopathology was $0.03 \mathrm{~mm}$. The mean difference between breast MRI and histopathology was $2.12 \mathrm{~mm}$. Using a $2 \times 2$ contingency Table to assess the frequency distribution of a relevant size discrepancy of $>1 \mathrm{~cm}$ between the two imaging modalities and histopathological results, we did not observe any advantage of performing an additional breast MRI after CESM in any of the cases.

Conclusion: Quality of tumour size measurement using CESM is good and matches the quality of these measurement assessed by breast MRI. Additional measurements using breast MRI did not improve the quality of tumour size measurements. 


\section{Introduction}

Contrast-enhanced spectral mammography (CESM, or contrast-enhanced dualenergy mammography: CEDM) is a promising new breast imaging modality. In CESM, an iodine-based contrast agent is intravenously administered two minutes prior to the acquisition of the mammographic images. These consist of a dualenergy technique which results in the acquisition of both a low- and high-energy image [1]. The low-energy image is similar to full-field digital mammography (FFDM) [2], whereas the high-energy image is used to generate a so-called 'recombined image', in which information on enhancing structures can be appreciated. A summary review of the most recent publications showed that the diagnostic accuracy of CESM in breast cancer detection was consistently superior to FFDM [3].

Breast MRI is currently regarded as the most accurate breast imaging modality available. Breast MRI is superior to all other imaging modalities for preoperative evaluation of breast cancer extent, since it assesses tumour diameter most accurately [4-6]. However, since breast MRI and CESM are based on similar principles, CESM might show a similar performance. Previously, only one study reported on the quality of tumour diameter measurements using both CESM and breast MRI [7]. In this paper the correlation of both measurements (with histopathological analysis as the gold standard) was presented. Studying the reproducibility of these results and more importantly the agreement between these two measurements provides additional information on the quality of these measurements [8].

In this study, our aims were two-fold: (1) to evaluate correlation and agreement of tumour size measurements using CESM, and (2) to evaluate whether measurements using an additional breast MRI exam would yield more accurate results.

\section{Materials and methods}

In our hospital, all women recalled from the breast cancer screening program who have no contra-indications for the intravenous administration of an iodine-based contrast agent are eligible for CESM (SenoBright*, GE Healthcare, United Kingdom). The need for obtaining written informed consent for this study was waived by Maastricht University Medical Center ethics committee.

The CESM imaging protocol has been described elsewhere in detail [9]. In short, a non-ionic monomeric low-osmolar contrast agent (iopromide; Ultravist $\AA 300$, Bayer Healthcare, Germany) is administered intravenously, after which CESM acquires a set of low and high energy images in quick succession. As a result, an image similar to a conventional mammogram (i.e. the low-energy CESM image) [2] and an image containing information on enhancement of structures can be reviewed (i.e. the recombined CESM image) (Figure 1).

All CESM exams performed between January 1st 2013 and April 1st 2014 were included. Out of this database, all consecutive breast cancer cases were collected, 
including ductal carcinoma in situ (DCIS). Since both low energy and recombined images are an integral part of the exam, maximum tumour diameter measurement is performed on the entire CESM exam (i.e. viewing both the low and recombined images together in both projection views) using callipers and a dedicated mammography workstation (IDI MammoWorkstation, GE Healthcare, United Kingdom). Core biopsies were performed immediately after the CESM exam.

In some cases, breast MRI was performed for preoperative staging, according to our national guidelines [10]. These indications for preoperative breast MRI were: women considering breast conserving therapy with either invasive lobular carcinoma, high grade ductal carcinoma in situ or size discrepancies between mammography and ultrasound $>1 \mathrm{~cm}$, and young women with dense breast tissue. All breast MRI exams were performed on a single 1.5 Tesla scanner (Intera, Philips Healthcare, the Netherlands) using a dedicated 16-channel breast coil. The protocol consisted of a transverse T2w sequence and diffusion weighted imaging (DWI), combined with a dynamic, contrast-enhanced T1w sequence in the transverse plane (consisting of $1.0 \mathrm{~mm}$ isotropic voxels and a temporal resolution of $93 \mathrm{sec}-$ onds). Blinded for CESM measurements, tumour diameter measurements were performed on the first dynamic scan after contrast administration, i.e. at peak enhancement of the tumour, using callipers and a dedicated workstation (Dynacad, Invivo International, the Netherlands). We recorded the presence of hematomas or other post-biopsy effects seen on breast MRI which might influence the accuracy of tumour size measurements.

After surgery, specimens were received fresh, routinely handled the pathology department, and tumour size was macroscopically measured in three dimensions. Histopathological examination was performed after formalin fixation and paraffin embedding. Hematoxillin and eosin stained sections were examined to assess tumour size. Microscopic carcinoma size was correlated with tumour size on gross examination. Tumour diameter (in $\mathrm{mm}$ ) was defined as largest tumour size based on macroscopic and histopathologic examination. For the comparison of maximum lesion diameter found using CESM and pathology, surrounding DCIS was included in the final maximum diameter measurement. In case of multifocal breast cancer, the maximum diameter of the largest invasive tumour (i.e. the primary index tumour) was assessed. 

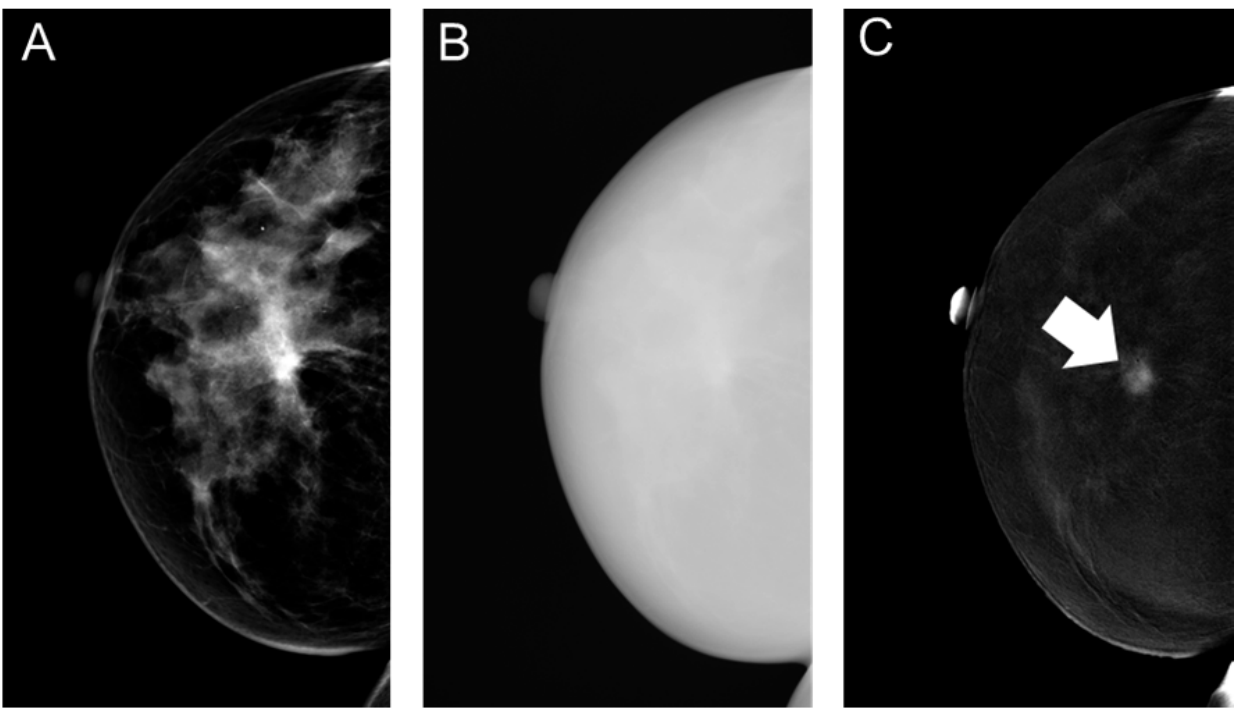

Figure 1. Typical example of the different contrast-enhanced spectral mammography images. First, a low-energy image is acquired (A), immediately followed by the high-energy image (B), which is used in post-processing to create the recombined image $(\mathrm{C})$, in which the invasive breast cancer is clearly visible (arrow).

\section{Statistical analysis}

The correlation between maximum tumour diameter based on CESM and histopathology, and breast MRI and histopathology was expressed using scatterplots and Pearson's correlation coefficient (PCC). In addition, the agreement between these measurements was expressed using Bland-Altman plots and calculating the mean difference in diameter between these measurements, including their $95 \%$ limits of agreement (LOA) [11].

A relevant size discrepancy was defined as a size difference of $>1 \mathrm{~cm}$ between CESM measurements and histopathological results, in line with previous publications $[12,13]$ and concordant with margins used by our surgeons. A $2 \times 2$ contingency Table was created for both imaging modalities and their corresponding size estimations. All statistical analyses were performed using SPSS Statistics (version 20.0, IBM, Armonk, NY, USA). All p-values $<0.05$ were considered statistically significant.

\section{Results}

In the study period, a total of 325 patients were referred to our hospital because of a suspicious abnormality detected in the breast cancer screening program. Of these, 87 (26.8\%) women were diagnosed with either an invasive breast cancer or 
DCIS, which is concordant with disease prevalence known from previous publications for this population [14]. Mean age (of all patients) was 62.4 years, range 4974 years. One patient had bilateral breast cancer, resulting in a total of 88 breast cancer lesions for the final analysis. For the additional analyses using breast MRI related data, 57 cases (58 lesions) were available (i.e. having both CESM and breast MRI exams performed within two weeks' time frame). Invasive ductal carcinoma was the most frequently observed cancer type (70.5\%), followed by DCIS (14.8\%) and invasive lobular carcinoma (10.2\%). Mean tumour diameter was $23.6 \mathrm{~mm}$, range 3 to $110 \mathrm{~mm}$. We did not observe any post-biopsy effects on breast MR images that interfered with the accuracy of tumour size measurements. Table 1 presents detailed patient characteristics

The correlations of maximum tumour diameter measurements between CESM and histopathology, and breast MRI and histopathology are presented in Figure 2. The PCC of CESM and histopathology was 0.905, p<0.0001. The PCC for the comparison between breast MRI and histopathology was slightly higher than the one for CESM: 0.915 , $\mathrm{p}<0.0001$.

The agreement of maximum tumour diameter measurement between CESM and histopathology, and breast MRI and histopathology is presented in Figure 3. The mean difference between CESM and histopathology was only $0.03 \mathrm{~mm}$, with 95\% LOA of -18.44 and $18.40 \mathrm{~mm}$, respectively. The mean difference between breast MRI and histopathology was $2.12 \mathrm{~mm}$, with 95\% LOA of -11.46 and 15.71 $\mathrm{mm}$. In short, MRI shows a slight systematic overestimation of the tumour diameter measured, whereas CESM does not. The 95\% LOA of breast MRI tumour measurements are smaller than those of CESM.

Table 1. Patient characteristics for the total population and the cases that underwent both CESM and breast MRI.

\begin{tabular}{lll}
\hline & Total & Additional MRI \\
\hline Age in years (range) & $62.4(49-74)$ & $62.2(49-74)$ \\
Number of breast cancers & 88 & 58 \\
Mean tumour diameter in mm (range) & $23.6(3-110)$ & $20.6(4-83)$ \\
Invasive ductal carcinoma & $70.5 \%$ & $75.9 \%$ \\
Ductal carcinoma in situ & $14.8 \%$ & $3.4 \%$ \\
Invasive lobular carcinoma & $10.2 \%$ & $13.8 \%$ \\
Invasive mucinous carcinoma & $2.3 \%$ & $1.7 \%$ \\
Invasive micropapillary carcinoma & $1.1 \%$ & $1.7 \%$ \\
Encapsulated papillary carcinoma & $1.1 \%$ & $1.7 \%$ \\
\hline
\end{tabular}

Table 2 shows a $2 \times 2$ contingency Table displaying the frequency distribution of the studied variables for all 58 lesions that were measured with CESM and breast MRI. In most cases (84.5\%), no size discrepancies were observed for both modalities. As is explained on a case-by-case basis below, the addition of breast MRI did not result in more accurate size estimations when compared to CESM. 

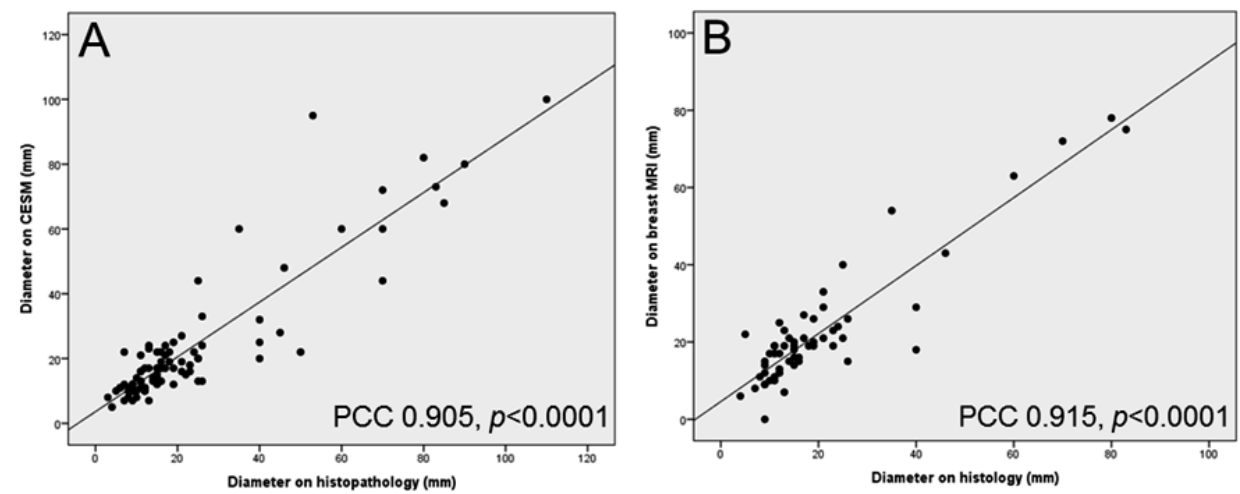

Figure 2. Scatterplots and Pearson's correlation coefficients (PCC) of maximum tumour diameter measurements between CESM and histopathology (A) and (B) breast MRI and histopathology.
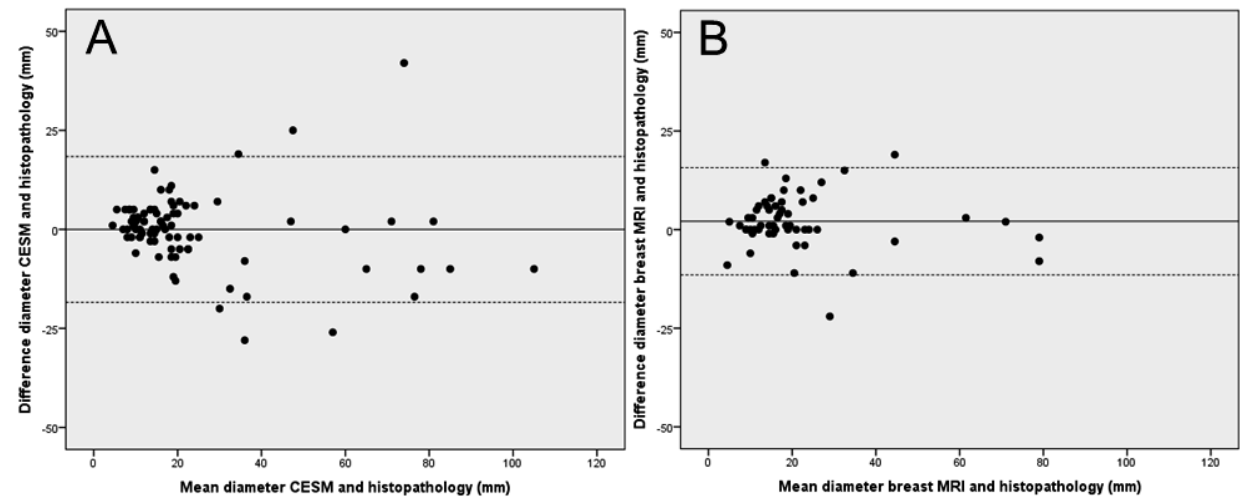

Figure 3. Bland-Altman plots for the comparison between (A) CESM and histopathology and (B) breast MRI and histopathology. Continuous lines represent the mean of the differences between measurements, the dotted lines represent upper and lower limits of 1.96 times the standard deviations of differences

Table 2. 2x2 contingency Table of tumour diameter measurement assessed by CESM or breast MRI

\begin{tabular}{llll}
\hline & $\begin{array}{l}\text { CESM diameter } \\
>1 \mathrm{~cm}\end{array}$ & $\begin{array}{l}\text { CESM diameter } \\
<1 \mathrm{~cm}\end{array}$ & Total \\
\hline $\begin{array}{l}\text { MRI diameter } \\
>1 \mathrm{~cm}\end{array}$ & $5(8.6 \%)$ & $3(5.2 \%)$ & $8(13.8 \%)$ \\
$\begin{array}{l}\text { MRI diameter } \\
<1 \mathrm{~cm}\end{array}$ & $1(1.7 \%)$ & $49(84.5 \%)$ & $50(86.2 \%)$ \\
Total & $6(10.3 \%)$ & $52(89.7 \%)$ & $58(100.0 \%)$ \\
\hline
\end{tabular}

A relevant size discrepancy was observed for both techniques simultaneously in 5 cases (all invasive ductal carcinomas: four grade II and one grade III carcinoma). In two cases, tumour size was overestimated by both modalities. A $25 \mathrm{~mm}$ carcinoma was estimated to be $44 \mathrm{~mm}$ on CESM and $40 \mathrm{~mm}$ on breast MRI. A $35 \mathrm{~mm}$ carcinoma was estimated to be $60 \mathrm{~mm}$ on CESM and $54 \mathrm{~mm}$ on breast MRI. In three cases, 
tumour size was underestimated by both modalities. In these cases, the subsequent diameters were 25, 20 and $13 \mathrm{~mm}$ for CESM and 29, 18 and $15 \mathrm{~mm}$ for breast MRI. Final histopathological analysis showed these tumours to be 40,40 and $26 \mathrm{~mm}$. Although underestimation of tumour size carries the risk of positive surgical margins, these were not observed in these three cases.

In 3 cases, no relevant size discrepancy was observed for CESM, whereas the addition of breast MRI resulted in an inaccurate size estimation. All cases were invasive ductal carcinomas (two grade I, one grade III carcinomas). All tumour sizes were overestimated by MRI $>1 \mathrm{~cm}$. For CESM, tumour diameters were 27, 17 and $10 \mathrm{~mm}$, respectively (versus 21,12 and $5 \mathrm{~mm}$ at histopathological examination). For breast MRI, these measurements were 33, 25 and $22 \mathrm{~mm}$, respectively. In these cases, CESM would already have estimated tumour size accurately and the addition of a breast MRI exam would increase the risk of unnecessary wider tumour excision.
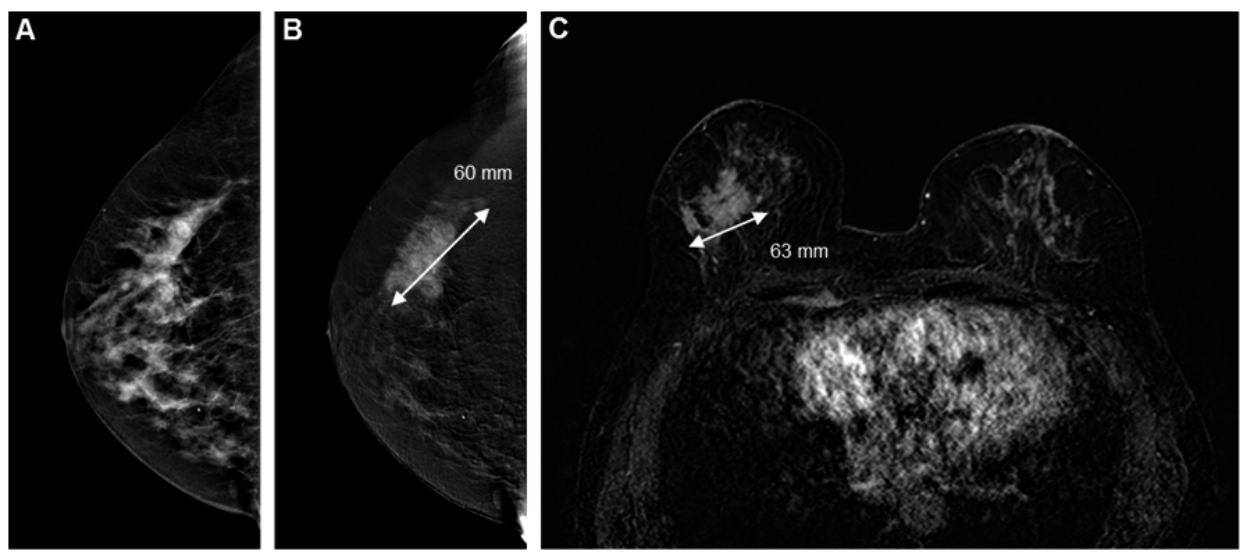

Figure 4. Image example of good agreement between tumour diameter measurements using CESM and breast MRI. The cancer is ill-defined on the low-energy CESM image (A) and can be measured more confidently on the recombined image (B, $60 \mathrm{~mm}$ ). Subtracted contrast-enhanced $\mathrm{T} 1 \mathrm{w}$ images (C) showed a similar irregular mass $(63 \mathrm{~mm})$. Final pathological results showed a $60 \mathrm{~mm}$ invasive ductal carcinoma

Only one case showed a potential benefit of the addition of breast MRI. This invasive lobular carcinoma's maximum diameter was $13 \mathrm{~mm}$. The diameters measured by CESM and breast MRI were 24 and $23 \mathrm{~mm}$, respectively. Per definition, this resulted in a relevant size discrepancy for CESM and not for breast MRI, but it is obvious that the addition of breast MRI did not have any clinical impact. In Figure 4 and 5, examples of good and poor agreement between tumour diameter measurement between CESM and breast MRI can be appreciated. 

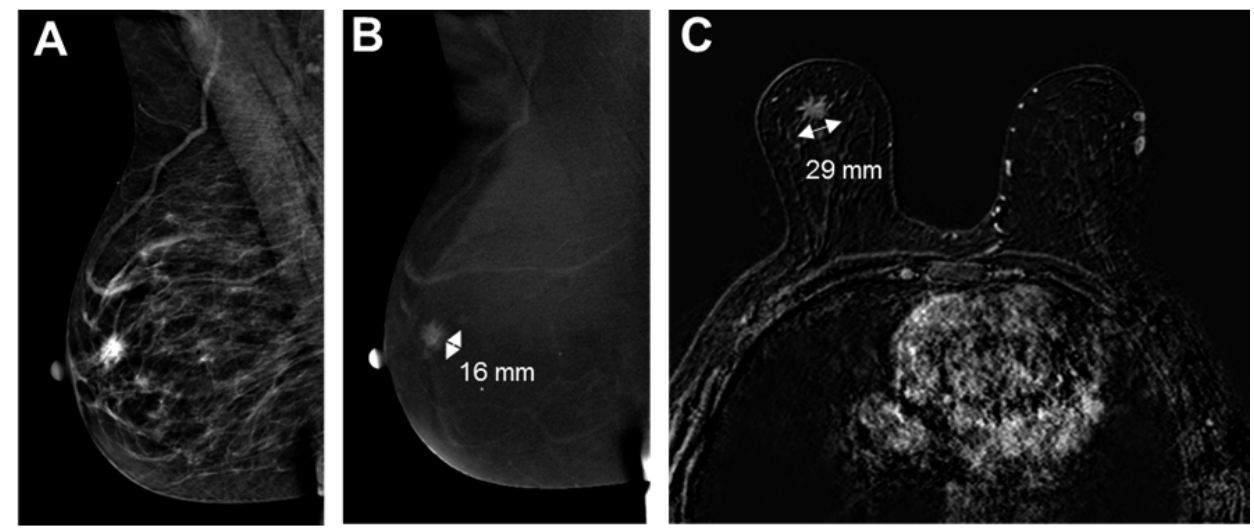

Figure 5. Example of poor agreement between CESM and breast MRI. The low-energy CESM image (A) shows an ill-defined mass behind the nipple, enhancing on the recombined images (B, $16 \mathrm{~mm}$ ). Breast MRI showed a speculated mass (C, $29 \mathrm{~mm}$ ). Histopathological results showed a $21 \mathrm{~mm}$ invasive lobular carcinoma.

\section{Discussion}

In this study, we evaluated the quality of maximum tumour diameter measurements of CESM with histopathologic results as gold standard. Where available, the quality of tumour diameter measurements as assessed by breast MRI was also studied. We demonstrated a good correlation and agreement between these measurements. A $2 \times 2$ contingency Table showed that the addition of a breast MRI exam after CESM did not yield more accurate tumour diameter measurements in any of the cases.

CESM is a new breast imaging tool that recently became commercially available. The CESM exam provides the radiologist with a so-called low-energy image (which is comparable to a regular mammogram) and a 'recombined' image, in which enhancing structures are visualized [15]. In a recent summary review it was shown that CESM was consistently superior to conventional full-field digital mammography [2]. However, these studies mainly focused on the ability of CESM to detect breast cancer, not on the quality of maximum tumour diameter measurements (which are essential for surgical planning).

There is only one prior publication that calculated PCC for CESM, breast MRI and histopathology [7]. In this study by Fallenberg et al., the PCC for CESM and histopathology was $0.733(\mathrm{p}<0.0001)$, and $0.654(\mathrm{p}<0.0001)$ for breast MRI and histopathology. In our study, PCC's were $0.905(\mathrm{p}<0.0001)$ and $0.915(\mathrm{p}<0.0001)$ respectively. However, merely calculating the PCC can be misleading, since good correlation does not automatically imply good agreement between measurements of two different modalities $[8,11]$. If there is a systematic over- or underestimation 
in the two measurements, PCC can be high, while agreement can be poor. For these comparisons, a Bland-Altman plot might be a more suitable method of presenting the results, since it allows us to evaluate the range and magnitude of measurement errors and shows whether these errors are acceptable in terms of clinical consequences [11]. As our Bland-Altman plots show, breast MRI shows a small tendency to overestimate tumour size (mean difference $+2 \mathrm{~mm}$ ), whereas CESM does not. However, the 95\% LOA of breast MRI are smaller than CESM. These plots also show that the observed mean differences are in our opinion clinically neglible.

In a $2 \times 2$ contingency Table, we studied the frequency distribution of relevant size discrepancies between measurements performed using CESM or breast MRI. A relevant size discrepancy was defined as $>1 \mathrm{~cm}$ between CESM tumour size measurements and the final histopathological tumour size measurement. This approach allowed us to study in the 58 lesions that were measured by both CESM and breast MRI if an additional breast MRI after a CESM exam would results in more accurate tumour size estimations. In the majority of cases, no relevant size discrepancies were observed in both modalities. In three cases, CESM already assessed tumour size accurately, whereas an additional breast MRI exam would result in a larger overestimation of tumour size, resulting in unnecessary excision of healthy breast tissue. In three cases, both CESM and breast MRI underestimated breast cancer size, but this did not result in tumour-positive surgical margins. Only one case of a $13 \mathrm{~mm}$ tumour showed a potential benefit of the addition of breast MRI, but this was mainly caused by our predefined cut off value for size discrepancy of $1 \mathrm{~cm}$. In short, this $2 x 2$ contingency Table did not show any benefit of additionally performing a breast MRI exam after CESM in any of the cases.

This study had several limitations. First, the sample size of the population is small, which refrained us from studying the ability of CESM to detect multifocal breast cancer in comparison to breast MRI. In a paper by Jochelson et al., CESM was compared to breast MRI with respect to cancer detection rates and false-positive findings, but also for the evaluation of multifocal breast cancer. In this study of 52 cancer cases, $88 \%$ of the additional ipsilateral breast cancers were detected by breast MRI, versus 56\% detected by CESM [16]. Consequently, breast MRI might be more accurate than CESM in detecting multifocal breast cancers. We could not perform any additional analysis on the ability of CESM to detect multifocal breast cancer ourselves due to the limited number of cases $(n=4)$. As a result, there is currently insufficient evidence that CESM can also be reliably used in multifocal breast cancers, and as such additional breast MRI should be considered if multifocal breast cancer is suspected.

Second, studying the agreement between maximum tumour diameter using imaging methods and comparing them to histopathological results has some inherent limitations. For example, tumour diameter may be distorted during the process of removal and fixation of the surgical specimen [17-20]. Due to the pliable nature of the breast, tumour diameter may vary depending on patient positioning 
during imaging exams [19]. In addition, orientation of intact specimens so that tumour diameters are measured in the similar planes in histologic analysis as in imaging might be challenging [17]. These potential errors in measurements are particularly important in retrospective analyses like our current study, since histopathological tumour size measurements were not performed for the purpose of this study.

Nonetheless, these promising results warrant larger studies which should be performed in multiple centres in order to include sufficient cases of especially multifocal breast cancers. In addition, these studies should also focus on the planned surgical treatment based on the different modalities and study how often the surgical management would be altered for the good or the worse. The results of this study show the potential of CESM as an alternative for tumour size measurements in hospitals with less access the MRI facilities.

In conclusion, the quality of tumour size measurement using CESM is good and the addition of a breast MRI exam for this purpose does not seem beneficial, unless multifocal breast cancer is suspected. CESM might be a suitable alternative for preoperative evaluation of tumour extent. Potential advantages of CESM over breast MRI are the short examination times, easy accessibility and less costs. Disadvantages are the use of an iodine based contrast agent instead of a gadolinium based agent and an increase in radiation dose as compared to FFDM [21]. However, if multifocal breast cancer is suspected, breast MRI should remain the preferred imaging modality, as there is still insufficient evidence that CESM is equally accurate as breast MRI to assess the extent of multifocal breast cancers. 


\section{References:}

1. Dromain C, Balleyguier C, Adler G, et al. Contrast-enhanced digital mammography. Eur J Radiol. 2009; 69: 34-42.

2. Francescone MA, Jochelson MS, Dershaw DD, et al. Low energy mammogram obtained in contrastenhanced digital mammography (CEDM) is comparable to routine full-field digital mammography (FFDM). Eur J Radiol 2014; 83: 1350-1355.

3. Lobbes MB, Smidt ML, Houwers J, et al. Contrast-enhanced mammography: techniques, current results, and potential indications. Clin Radiol. 2013; 68: 935-944.

4. Van Goethem M, Tjalma W, Schelfout K, et al. Magnetic resonance imaging in breast cancer. Eur J Surg Oncol. 2006; 32: 901-910.

5. Sardanelli F, Boetes C, Borisch B, et al. Magnetic resonance imaging of the breast: recommendations from the EUSOMA working group. Eur J Cancer 2010; 46: 1296-1316.

6. Ramirez SI, Scholle M, Buckmaster J, et al. Breast cancer tumour size assessment with mammography, ultrasonography, and magnetic resonance imaging at a community based multidisciplinary breast center. American Surgeon. 2012; 78: 440-446.

7. Fallenberg EM, Dromain C, Diekmann F, et al. Contrast-enhanced spectral mammography versus MRI: initial results in the detection of breast cancer and assessment of tumour size. Eur Radiol. 2014; 24: 256-264.

8. Lobbes MB, Nelemans PJ. Good correlation does not automatically imply good agreement: the trouble with comparing tumour size by breast MRI versus histopathology. Eur J Radiol. 2013; 82: e906-907.

9. Lobbes MB, Lalji U, Houwers J, et al. Contrast-enhanced spectral mammography in patients referred from the breast cancer screening programme. Eur Radiol. 2014; 24: 1668-1676.

10. Nationaal Borstkanker Overleg Nederland (NABON). National guideline breast cancer 2012. Amsterdam, the Netherlands: NABON; 2012.

11. Bland JM, Altman DG. Statistical methods for assessing agreement between two methods of clinical measurement. Lancet. 1986; 1: 307-310.

12. Schouten van der Velden AP, Boetes C, Bult P, Wobbes T. Magnetic resonance imaging in size assessment of invasive breast carcinoma with an extensive intraductal component. BMC Med Imaging 2009; 9: 5 .

13. Mann RM, Bult P, Van Laarhoven HW, et al. Breast cancer size estimation with MRI in BRCA mutation carriers and other high risk patients. Eur J Radiol 2013; 82: 1416-1422.

14. Timmers JM, Van Doorne-Nagtegaal HJ, Zonderland HM, et al. The Breast Imaging Reporting and Data System (BI-RADS) in the Dutch breast cancer screening programme: its role as an assessment and stratification tool. Eur Radiol. 2012; 22: 1717-1723.

15. Jochelson M. Contrast-enhanced digital mammography. Radiol Clin N Am. 2014; 52: 609-616.

16. Jochelson MS, Dershaw DD, Sung JS, et al. Bilateral contrast-enhanced dual-energy digital mammography: feasibility and comparison with conventional digital mammography and MR imaging in women with known breast cancer. Radiology. 2013; 266: 743-751.

17. Provencher L, Dioro C, Hogue JC, et al. Does breast cancer tumour size really matter that much? Breast. 2012; 21: 682-685.

18. Behjatnia B, Sim J, Bassett LW, et al. Does size matter? Comparison study between MRI, gross, and microscopic tumour sizes in breast cancer in lumpectomy specimens. Int J Clin Exp Pathol. 2010; 22: 303-309.

19. Pritt B, Tessitore JJ, Weaver DL, et al. The effect of tissue fixation and processing on breast cancer size. Hum Pathol. 2005; 36: 756-760.

20. Tucker FL. Imaging-assisted large-format breast pathology: program rationale and development in a non-profit health system in the United States. Int J Breast Cancer. 2012; 171792.

21. Jeukens CR, Lalji UC, Meijer E, et al. Radiation exposure of contrast-enhanced spectral mammography compared to full field digital mammography. Invest Radiol 2014; 49: 659-665. 


\section{Discussion}





\section{Discussion}

With breast cancer being one of the most important cancer related causes of death in women worldwide, mammography continues to play a central role in detection and evaluation [1].

An important limitation of mammography is its limited diagnostic performance in dense breasts as Carney et al. demonstrated in their study based on 329,495 women of which 2,223 were diagnosed with breast cancer) [2]. They found that in breasts consisting almost entirely of fat sensitivity was $88.2 \%$ and specificity $96.5 \%$ and in extremely dense breasts, sensitivity dropped to $62.2 \%$ and specificity to $89.9 \%$ [2].

In the last years novel techniques were developed to overcome this limitation. Contrast-enhanced spectral mammography (CESM) is one of the latest developments in advanced mammography using intravenously administered iodine-based contrast agents.

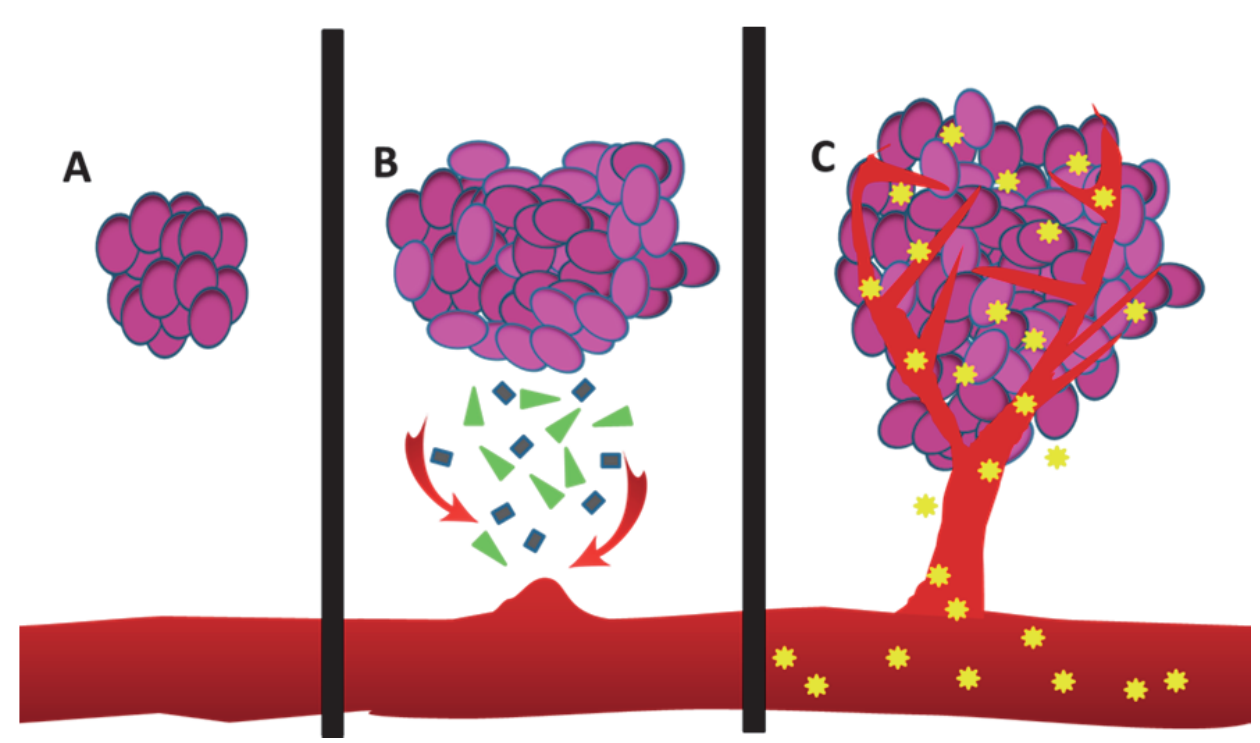

Figure 1: Neoangiogenesis. When a cluster of tumour cells (A) reaches a certain size it needs more nutrients to grow. By releasing proangiogenic factors new vessels are induced (B). These vessels have an abnormal endoepithelial layer which enables contrast agents (yellow stars) to leak into the tumour interstitium causing enhancement $(\mathrm{C})$.

There is a reason why contrast can be used in cancer imaging. As tumours develop the demand for nutrients outgrows the levels supplied by regular diffusion mechanisms. By releasing proangiogenic factors new vessels are formed in a process called neoangiogenesis in order to fulfil the increased demand of nutrients and oxygen. However, the endothelial cells of these new vessels are 'leaky', enabling particles such as contrast media to extravasate into the tumour interstitium (Figure 1) [3]. This principle is well known from dynamic contrast-enhanced (DCE) 
Magnetic Resonance Imaging (MRI) and DCE-computed tomography (CT) [4]. The enhancement of breast abnormalities in breast MRI after the administration of a Gadolinium-based contrast agent accounts for its high sensitivity [5, 6]. CESM (using the same Iodine based contrast agents as DCE-CT) is based on similar principles.

The first studies using CESM were published from 2003 onwards, mostly consisting of pilot studies using prototype units in study populations predominantly consisting of breast cancers and a relatively low number of benign entities (if any) [7$11]$.

In short, these studies showed that CESM was feasible and showed promising initial results for cancer detection. In an attempt to reproduce the signal intensity time curves as used in breast MRI [8], a temporal approach was studied in which CESM was performed a certain time points after contrast administration. Probably due to differences in contrast pharmacokinetics and the volume of contrast injected, these did not prove to be reproducible for CESM and were abandoned for the time being.

In 2011, Dromain et al. were the first to publish the results of CESM in a clinical population [12]. In this study, the CESM protocol that is still in use today was presented, in which both breasts can be imaged in both standard mammographic views, while elevating breast compression between image acquisitions, thus reducing the number of motion artefacts.

CESM became commercially available as a breast imaging tool in 2010 and was approved by the Food and Drug Administration (FDA) in 2011 as an adjunct to mammography and ultrasound [13]. However, in order to become fully accepted as a breast imaging tool, several important factors needed to be studied, such as radiation dose, image quality, and preliminary diagnostic performance.

\section{Radiation dose}

For clinical use radiation dose must meet the demands as set by internationally acceptable standards. Previously, only a few studies reported on the radiation dose of CESM in relation to FFDM examinations. However, the reported radiation doses varied, with one study observing an almost equal radiation dose between mammography and CESM [14], whereas other studies reported a dose increase of $20 \%$ [13] and even a $54 \%$ increase [14]. None of these studies performed phantom measurements to validate the radiation dose that was calculated by the unit itself.

In chapter 3 we validated the dose values provided by the CESM unit by performing phantom measurements. After assuring that there were no relevant differences in the values provided by the machine and the phantom measurements, we were able to use clinical exams to further assess the different dose levels used in the different exams in different breasts. For CESM, we found a mean ESAK of 
10.5 mGy and a mean AGD of 2.80 mGy, representing an increase of 3.07 mGy (41\%) and $1.25 \mathrm{mGy}(81 \%)$ respectively when compared to our institutional FFDM dose. Although the medical benefit must outweigh the risk associated with an increased radiation dose, we showed that this added dose is extremely small and well within the limits set by the European Reference Organisation for Quality Assured Breast Screening and Diagnostic Services (EUREF) [15].

\section{Image quality}

Until now, CESM was considered as an adjunct imaging modality next to FFDM. A CESM exam consists of a high energy image used for the generation of a recombined image (showing areas of enhancement) and a low energy image (LE) which resembles a normal mammogram. If LE images are equal to FFDM, CESM could also be considered as a primary imaging tool in patients with a direct CESM indication, such as a suspicious palpable mass, when screening for an unknown primary tumour, as part of the workup of a detected abnormality in the breast on other imaging modalities (such as a chest CT) or in patients who undergo screening prior to preventive mastectomy. The settings for $\mathrm{kV}$ and mAs used in both FFDM and LE CESM images are comparable, suggesting that image quality should also be comparable.

Only two prior studies were published studying this topic. Fallenberg et al. focused on cancer detection and size estimation of bilateral CESM, digital mammography, and the combination of CESM and mammography in 107 patients [16]. Even though cancer detection and size estimation are valid diagnostic imaging parameters, they do not reflect image quality criteria. A second study published by Francescone et al. focused on technical parameters including posterior nipple line distance, compression thickness, and compression force on the MLO projection [17]. Although both studies did not observe a significant difference between LE images in CESM and FFDM, well-validated and standardized image quality criteria such as suggested by EUREF were not used [15].

EUREF has defined a set of twenty image quality criteria that ensure a high quality of care concerning screening in detection of breast cancer using mammography. In chapter 4 we showed that the majority of EUREF image quality criteria showed neither clinically relevant nor statistically significant differences between low-energy CESM and FFDM exams. Interestingly, we found that visualization of micro calcifications was slightly superior in LE images. This might imply an improved detection of ductal carcinoma in situ (DCIS) with LE images, which should be studied more extensively in the future.

Reviewing the currently available scientific evidence and the results published in this thesis we can safely conclude that LE images obtained as part of a CESM exam are equal to FFDM images. Consequently, FFDM can be omitted if CESM is indicated. 


\section{Preliminary diagnostic performance}

When (after an initial pilot study) new techniques are introduced into everyday clinical practice, their preliminary results should be checked by comparing them to the present standard of care to guarantee the previously provided level of health care and to reveal any potential pitfalls. Furthermore, reproducibility of results is important for any diagnostic test, since widespread implementation in clinical practices can only be performed if results are reproducible between radiologists of various experience and various centres.

Several initial clinical studies showed that the diagnostic performance of CESM was consistently superior to that of mammography, thus showing its the potential of CESM as an accurate breast imaging modality [12, 18-20]. Nonetheless, the number of patients in these studies were limited (mean 87.3, range 72 to 110 patients). There was a high prevalence of malignancy in these populations ranging from $57 \%$ to $100 \%$ malignant lesions and performance in populations with lower disease prevalence was not yet determined. If CESM would generate a large number of false positives in populations with low breast cancer prevalence its added clinical value would be diminished.

For this reason we decided to perform CESM in women recalled from the breast cancer screening, whom have a breast cancer prevalence of approximately $30 \%$, allowing us to study the performance of CESM in a study population with a much lower disease prevalence. In the first six months, we performed CESM next to our regular work-up (which is according to the current Dutch national guidelines on breast cancer) of these women. In chapter 5 we performed an interim analysis after these six months. Eligible patients were screened using a questionnaire based on the guidelines of the European society of Urogenital Radiology (ESUR) to exclude those at risk of developing CIN [21]. Using this approach, 113 out of 116 (98\%) referred patients were eligible to undergo CESM. We observed a higher diagnostic accuracy of CESM compared to conventional mammography in this population. Sensitivity for CESM reached 100\% (FFDM 96.9\%), specificity reached 87.7\% (FFDM 42\%), negative predictive value (NPV) was 100\% (FFDM 97.1\%) and positive predictive value (PPV) was $76.2 \%$ for CESM (FFDM 39.7\%), respectively. We showed that CESM is not only clinically feasible but is also an excellent problem solving tool in women recalled from screening. CESM can confidently detect recalls based on superposition of fibroglandular tissue or cysts. It has the potential to replace all conventional imaging techniques for the work-up of recalls from screening (such as mammography and ultrasound).

As a next step, we validated the use of CESM in this population in chapter 6 by studying the reproducibility of the initial results in chapter 5. Mean sensitivity of all 10 readers for CESM was 96.9\% (FFDM 93.0\%) and mean specificity was $69.7 \%$ (FFDM 35.9\%). In short, even when more radiologists with varying CESM- 
experiences were assessing the images, CESM again showed its robustness as a problem-solving tool in recalls from the breast cancer screening program.

In addition, breast MRI is currently considered to be the most accurate imaging modality available in detecting breast cancers with a specificity of $67 \%$ and a sensitivity of 95\% [22]. Regarding tumour size estimation breast MRI is considered to be superior to ultrasound and mammography [23]. In a study in preoperative patients by Wendle et al., MRI was found to be superior to mammography and ultrasound, not only showing a higher sensitivity but also detecting additional tumours that were previously [24].

Since the principle of breast MRI and CESM is based on visualising neoangiogenesis, comparing CESM and MRI in detection of lesions and measurement of tumour extent would be a logical step in determining the value of CESM. In a study by Fallenberg et al. mammography, CESM and MRI were compared regarding the detection and size estimation of proven breast malignancies [20]. Besides proving to be superior over mammography, CESM also had a higher sensitivity than breast MRI . Compared to histopathology, no significant difference in tumour size measured on CESM and MRI was observed. In chapter 7 we compared maximum tumour size measurements of 58 lesions using CESM and MRI as compared to histopathology. We observed a good correlation and agreement between both techniques with respect to disease extent assessment. Compared to CESM MRI showed a slight overestimation of disease extent. In a sub analysis, we also showed that in patients who underwent both CESM and MRI that concerning disease extent the latter did not result in relevant additional preoperative information.

All studies published so far on this topic showed that CESM might have an at least similar performance compared to breast MRI, with equal sensitivity and potentially improved specificity. These first studies also suggested that preoperative evaluation using CESM might be equally accurate. However, only limited information is available on the detection of multifocal cancer using CESM with one publication showing more accurate disease evaluation in multifocal breast cancers using breast MRI [19]. Consequently, if multifocal breast cancers are suggested on CESM or other conventional imaging methods breast MRI should still be performed.

\section{Future perspectives}

Currently, studies demonstrate that CESM is consistently superior to FFDM, even when the latter is combined with breast ultrasound. The diagnostic performance of CESM even seems to match the performance of breast MRI. Even so, these results are based on studies using relatively small study populations with relatively high disease prevalence. The next step in studying CESM needs to be the evaluation of proper CESM indications in prospective trials. In addition, the potential of CESM to replace breast MRI or conventional mammography for certain indications needs to be studied in large, multicentre randomized controlled trials. Future studies com- 
paring CESM and breast MRI should also focus on the cost-effectiveness of using CESM instead of breast MRI in various study populations.

At present, there is no standardized terminology to describe the abnormalities found at CESM exams. Although CESM consists of a 'mammography' component, the enhancement characteristics observed on the recombined images differ from those observed in breast MRI exams. As a result, the terminology for reporting mammography and MRI exams (as dictated in the BI-RADS lexicon) cannot be simply copied to CESM. The development of a specific CESM lexicon seems mandatory to ensure structured reporting, enabling comparison of multiple studies in meta-analyses and systematic reviews. CESM-specific descriptors for degrees of enhancement, artefacts and lesions with specific CESM appearance (such as the 'eclipse sign' suggesting an underlying cyst) should be defined. These descriptors should also be further evaluated in large, prospective studies between different centres, preferably using multiple readers and multiple vendors. The final step would be to incorporate the CESM-specific descriptors in the BI-RADS lexicon.

Furthermore, CESM's radiation dose is higher compared to FFDM for which the LE images are responsible. Since the construction of the recombined images are dependent on the LE and high energy images, lowering the dose of the LE images could potentially influence the appearance of the recombined images. In an attempt to optimize CESM, Dromain et al. identified 4 clinical indications that might justify the use of a different AGD: establishing disease extent with CESM, as problem solver after inconclusive findings, screening of high risk women and finally CESM guided biopsy [25]. The rationale behind different AGD is that for different indications different degrees of image quality could suffice therefore resulting in a dose reduction. These kinds of developments might decrease the dose needed for these exams. For further dose reduction one could also argue that perhaps one view might be sufficient, for certain indications, such as screening high risk women, thus reducing radiation dose even more.

An important limitation of a CESM exam is that CESM guided interventions are not (yet) available. Hypothetically, the recombined images of CESM could show enhancing lesions for which there is no mammographic or sonographic substrate. In current clinical practice a work-around exists: the enhancing lesion on CESM is most likely visible on breast MRI as well, enabling MRI guided biopsy. However, this delays the diagnostic process, since a diagnostic breast MRI has to be performed first in order to plan a breast MRI guided intervention. If CESM-guided biopsy would be available it would prevent this labour-intensive work-around and enable institutions without access to breast MRI-guided biopsies to evaluate these lesions with CESM.

An interesting recent development is spectral contrast-enhanced digital breast tomosynthesis (CE-DBT). This method combines CESM with breast tomosynthesis. In breast tomosynthesis the compressed breast is imaged in multiple planes, resulting in three dimensional imaging volumes, usually in $1 \mathrm{~mm}$ slices [26]. Several large recent studies showed that especially in screening settings breast tomosynthesis increases cancer detection rate while lowering the number of false-positive 
recalls compared to FFDM [27-29]. Several CE-DBT prototype machines are currently being investigated. This approach combining two advanced mammography techniques would appear as the 'best of both worlds'. Recently, Froeling et al. demonstrated that with CE-DBT, signal intensity time curves might indeed be evaluated [30]. In their pilot study they included 21 patients with a total of 17 malignancies, CE-DBT was acquired in MLO view consisting of 19-24 3-mm slices before contrast administration, at 2 and 8 minutes after contrast administration. Despite significant concordant agreement of contrast enhancement curves between spectral CE-DBT and CE-MRI (Spearman's rho $=0.49$ ) there was moderate agreement (Cohen's kappa $=0.438$ ). Since these results are based on a single pilot study, future studies should evaluate the added value of tomosynthesis to CESM.

\section{Conclusion}

CESM is a new modality in the detection, characterization and follow up of breast lesions with promising results that justifies it use in daily clinical practice. It has been shown to be consistently superior to mammography with or without targeted ultrasound and matches the diagnostic accuracy of breast MRI. Full-field digital mammography can be omitted if a direct indication for CESM exists. Although radiation dose of CESM is increased by approximately $80 \%$, this increased dose is still within internationally accepted standards. Further studies should not only further explore the diagnostic potentials of CESM but also focus on cost-effectiveness as well as further optimization of image quality and dose reduction. 


\section{References}

1. Ferlay J, Soerjomataram I, Ervik M, Dikshit R, Eser S, Mathers C, Rebelo M, Parkin DM, Forman D, Bray F GLOBOCAN 2012 v1.0, Cancer Incidence and Mortality Worldwide: IARC CancerBase No. 11 [Internet]. Lyon, France: International Agency for Research on Cancer; 2013. Available from: http://globocan.iarc.fr. http://globocan.iarc.fr/Pages/fact_sheets_cancer.aspx. Accessed 7 Mar 2015

2. Carney PA, Miglioretti DL, Yankaskas BC, et al. (2003) Individual and combined effects of age, breast density, and hormone replacement therapy use on the accuracy of screening mammography. Ann Intern Med 138:168-75.

3. Hashizume H, Baluk P, Morikawa S, et al. (2000) Openings between defective endothelial cells explain tumor vessel leakiness. Am J Pathol 156:1363-80. doi: 10.1016/S0002-9440(10)65006-7

4. Cuenod CA, Balvay D (2013) Perfusion and vascular permeability: basic concepts and measurement in DCE-CT and DCE-MRI. Diagn Interv Imaging 94:1187-204. doi: 10.1016/j.diii.2013.10.010

5. Leach MO (2001) Application of magnetic resonance imaging to angiogenesis in breast cancer. Breast Cancer Res 3:22-7.

6. Heywang SH, Wolf A, Pruss E, et al. (1989) MR imaging of the breast with Gd-DTPA: use and limitations. Radiology 171:95-103. doi: 10.1148/radiology.171.1.2648479

7. Lewin JM, Isaacs PK, Vance V, Larke FJ (2003) Dual-energy contrast-enhanced digital subtraction mammography: feasibility. Radiology 229:261-8. doi: 10.1148/radiol.2291021276

8. Kuhl CK, Mielcareck P, Klaschik S, et al. (1999) Dynamic Breast MR Imaging: Are Signal Intensity Time Course Data Useful for Differential Diagnosis of Enhancing Lesions? Radiology 211:101-110.

9. Diekmann F, Bick U (2007) Tomosynthesis and contrast-enhanced digital mammography: recent advances in digital mammography. Eur Radiol 17:3086-92. doi: 10.1007/s00330-007-0715-X

10. Diekmann F, Diekmann S, Jeunehomme F, et al. (2005) Digital mammography using iodine-based contrast media: initial clinical experience with dynamic contrast medium enhancement. Invest Radiol 40:397-404.

11. Dromain C, Balleyguier C, Muller S, et al. (2006) Evaluation of tumor angiogenesis of breast carcinoma using contrast-enhanced digital mammography. AJR Am J Roentgenol 187:W528-37. doi: 10.2214/AJR.05.1944

12. Dromain C, Thibault F, Muller S, et al. (2011) Dual-energy contrast-enhanced digital mammography: initial clinical results. Eur Radiol 21:565-74. doi: 10.1007/s00330-010-1944-y

13. http://www.accessdata.fda.gov/cdrh_docs/pdf10/k103485.pdf. http://www.accessdata.fda.gov/cdrh_docs/pdf10/k103485.pdf. Accessed 10 0ct 2015

14. Badr S, Laurent N, Régis C, et al. (2014) Dual-energy contrast-enhanced digital mammography in routine clinical practice in 2013. Diagn Interv Imaging 95:245-58. doi: 10.1016/j.diii.2013.10.002

15. EUREF European Guidelines - EUREF | European Reference Organisation for Quality Assured Breast Screening and Diagnostic Services.

16. Fallenberg EM, Dromain C, Diekmann F, et al. (2014) Contrast-enhanced spectral mammography: Does mammography provide additional clinical benefits or can some radiation exposure be avoided? Breast Cancer Res Treat 146:371-81. doi: 10.1007/s10549-014-3023-6

17. Francescone MA, Jochelson MS, Dershaw DD, et al. (2014) Low energy mammogram obtained in contrast-enhanced digital mammography (CEDM) is comparable to routine full-field digital mammography (FFDM). Eur J Radiol 83:1350-5. doi: 10.1016/j.ejrad.2014.05.015

18. Dromain C, Thibault F, Diekmann F, et al. (2012) Dual-energy contrast-enhanced digital mammography: initial clinical results of a multireader, multicase study. Breast Cancer Res 14:R94. doi: 10.1186/bcr3210

19. Jochelson MS, Dershaw DD, Sung JS, et al. (2013) Bilateral contrast-enhanced dual-energy digital mammography: feasibility and comparison with conventional digital mammography and MR imaging in women with known breast carcinoma. Radiology 266:743-51. doi: 10.1148/radiol.12121084

20. Fallenberg EM, Dromain C, Diekmann F, et al. (2013) Contrast-enhanced spectral mammography versus MRI: Initial results in the detection of breast cancer and assessment of tumour size. Eur Radiol. doi: 10.1007/s00330-013-3007-7 
21. Stacul F, van der Molen AJ, Reimer P, et al. (2011) Contrast induced nephropathy: updated ESUR Contrast Media Safety Committee guidelines. Eur Radiol 21:2527-41. doi: 10.1007/s00330-0112225-0

22. Hrung JM, Sonnad SS, Schwartz JS, Langlotz CP (1999) Accuracy of MR imaging in the work-up of suspicious breast lesions: a diagnostic meta-analysis. Acad Radiol 6:387-97.

23. Gruber I V, Rueckert M, Kagan KO, et al. (2013) Measurement of tumour size with mammography, sonography and magnetic resonance imaging as compared to histological tumour size in primary breast cancer. BMC Cancer 13:328. doi: 10.1186/1471-2407-13-328

24. Berg WA, Gutierrez L, NessAiver MS, et al. (2004) Diagnostic accuracy of mammography, clinical examination, US, and MR imaging in preoperative assessment of breast cancer. Radiology 233:83049. doi: $10.1148 /$ radiol.2333031484

25. Dromain C, Canale S, Saab-Puong S, et al. (2014) Optimization of contrast-enhanced spectral mammography depending on clinical indication. J Med imaging (Bellingham, Wash) 1:033506. doi: 10.1117/1.JMI.1.3.033506

26. Houssami N, Skaane P (2013) Overview of the evidence on digital breast tomosynthesis in breast cancer detection. Breast 22:101-8. doi: 10.1016/j.breast.2013.01.017

27. Houssami N, Skaane P (2013) Overview of the evidence on digital breast tomosynthesis in breast cancer detection. Breast 22:101-8. doi: 10.1016/j.breast.2013.01.017

28. Skaane P, Bandos AI, Gullien R, et al. (2013) Prospective trial comparing full-field digital mammography (FFDM) versus combined FFDM and tomosynthesis in a population-based screening programme using independent double reading with arbitration. Eur Radiol 23:2061-71. doi: 10.1007/s00330-013-2820-3

29. Gilbert FJ, Tucker L, Gillan MGC, et al. (2015) Accuracy of Digital Breast Tomosynthesis for Depicting Breast Cancer Subgroups in a UK Retrospective Reading Study (TOMMY Trial). Radiology 142566. doi: 10.1148/radiol.2015142566

30. Froeling V, Diekmann F, Renz DM, et al. (2013) Correlation of contrast agent kinetics between iodinated contrast-enhanced spectral tomosynthesis and gadolinium-enhanced MRI of breast lesions. Eur Radiol 23:1528-36. doi: 10.1007/s00330-012-2742-5 

Summary 
Breast cancer remains one of the leading causes cancer related deaths in women worldwide. In breast cancer detection, management and follow-up mammography continues to play a central role, but its diagnostic accuracy is largely dependent on breast density. Several techniques have been developed in the past to overcome this limitation. Contrast enhanced spectral mammography (CESM) is one of the latest technical achievements in this respect. The aim of this thesis is to explore the feasibility of clinical implementation of CESM and its accuracy in clinical practice, with focus on women recalled from a national breast cancer screening program.

In chapter 2 an introduction to CESM is presented, explaining its technical aspects and the consecutive imaging protocol as well as an overview of preliminary results performed with a prototype unit. These were very promising and consistently superior to full-field digital mammography (FFDM). However, these studies were performed in populations with a relatively high prevalence of malignancy. If CESM would result in a high number of false positive findings, the technique would still be regarded as unpractical in daily clinical practice. Therefore, we studied the accuracy of CESM in populations with a lower prevalence of breast cancer, such as recalled women from breast cancer screening. The next step was to evaluate if CESM would be clinically feasible, without loss of image quality or adding unacceptably high radiation dose.

We studied the radiation dose used in CESM in chapter 3. Previously, CESM radiation dose varied from being equal to FFDM to being increased by more than $50 \%$ compared to FFDM. We systematically validated the dose value provided by the unit by using phantom measurements and found they were correspondent. Subsequently, we showed that radiation dose was increased by $81 \%$ compared to FFDM for clinical images, using the commercially available unit. We therefore recommended the use of absolute dose values (in mGy) for the comparison of CESM and FFDM, rather than presenting relative numbers in \%. Although radiation dose used in CESM remains within internationally accepted limits, dose should always be outweighed to clinical relevance, especially in young women where MRI is an alternative.

In chapter 4 we studied image quality criteria of the low energy images produced by CESM compared to full field digital mammography. Low energy images were obtained from women that were recalled from the breast cancer screening program and matched one by one to their full field digital mammography images obtained during breast cancer screening just a few weeks earlier. Using image quality criteria defined by the European reference organisation for quality assured breast screening and diagnostic services (EUREF) we showed that not only are lowenergy images equal to FFDM but calcifications were better visualised on low energy images. The delineation of the pectoral muscle was less accurate in low energy CESM images, but presumably does not result in clinical dilemmas. Additional phantom measurement were performed to understand the underlying cause for 
these observations. Differences were most likely caused by differences in the postprocessing image algorithms, as no significant differences were subsequently detected by thorough analysis. As a result, we concluded that FFDM can be omitted if there is a direct indication for CESM.

In chapter 5 we performed an interim analysis of the diagnostic performance of CESM in 113 patients recalled from the breast cancer screening program in the first six months after CESM installation. Only three of 116 potentially eligible women did not undergo CESM for various reasons. For this population, sensitivity and negative predictive value using CESM were 100\% (FFDM 96.9\% and 97.1\%, respectively). The most significant increases were observed for specificity and positive predictive value, where CESM showed a specificity of $87.7 \%$ (FFDM $42.0 \%$ ) and a positive predictive value of $76.2 \%$ (FFDM 39.7\%). In conclusion, CESM is not only feasible in the clinical setting, it also a promising problem solving tool' for women recalled from breast cancer screening.

Evaluating the reproducibility of initial results in a larger group of radiologist is an important step in the introduction of every new imaging modality. In chapter 6, we therefore evaluated the diagnostic accuracy of CESM in a 199 cases, now read by a total of 10 readers. Four readers were experienced CESM users, three readers were experienced mammography readers without previous CESM experience and three readers were residents with limited training in CESM and mammography. Sensitivity, specificity, positive predictive value and area under the ROC curve increased in a trend similar to the results of the interim analysis in chapter 5 , regardless of reader experience with CESM. Also, inter-observer agreement turned out to be excellent. We further performed a sub analysis of the number and causes of both false positives and false findings. Of the 199 cases, only three cases were scored false negative by more than one reader. Further analysis revealed that the location of two of these lesions were on the edges of the image. The third lesion was a mucinous carcinoma which can also show little to no enhancement on MRI. These tumour types are a potential pitfall of CESM. The most frequent cause of CESM induced false positive findings were fibroadenomas.

In chapter 7 we assessed the ability of CESM to assess true breast cancer extent (with histopathological results as the gold standard) and additionally compared the findings to the extent assessed on breast MRI (if available). We determined both correlation and agreement between CESM, histopathology and MRI as good. In the future, breast MRI might be omitted for preoperative evaluation after performing CESM in breast cancer patients.

In chapter 8, the results of this thesis are discussed and an outlook on future developments are presented. 

Samenvatting 
Wereldwijd blijft borstkanker bij vrouwen een van de belangrijkste oorzaken van kanker gerelateerde sterfte. Mammografie blijft bij de detectie, behandeling en follow-up een centrale rol spelen, maar de diagnostische nauwkeurigheid is grotendeels afhankelijk van de dichtheid van de borstklierweefsel. Verscheidene technieken zijn in het verleden ontwikkeld om deze beperking te overwinnen. Contrast-versterkte spectrale mammografie (CESM) is een van deze nieuwste technieken. Het doel van dit proefschrift is om de klinische implementatie en de nauwkeurigheid in de klinische praktijk van CESM te verkennen, met de nadruk op vrouwen die zijn teruggeroepen uit het landelijk bevolkingsonderzoek naar borstkanker.

Hoofdstuk 2 is een inleiding tot CESM. Zowel de technische aspecten en het onderzoeksprotocol, alsmede een overzicht van de voorlopige resultaten (uitgevoerd met een prototype toestel) passeren de revue. Deze resultaten waren veelbelovend en de uitkomsten toonden consequent aan dat CESM superieur is aan conventionele- oftewel full-field digital mammography (FFDM). Deze studies werden echter verricht bij populaties met een relatief hoge borstkanker prevalentie. Als het gebruik van CESM een groot aantal vals positieve bevindingen zou genereren, moet de techniek toch worden beschouwd als onpraktisch in de dagelijkse klinische praktijk. Daarom bestudeerden we de accuratesse van CESM in een populatie met een lagere borstkanker prevalentie, namelijk vrouwen verwezen vanuit het landelijke bevolkingsonderzoek naar borstkanker. Ook wilden we evalueren of CESM in de klinisch setting bruikbaar is, zonder dat er sprake is van verlies van beeldkwaliteit of onaanvaardbaar hoge stralingsdosis.

We bestudeerden de stralingsdosis van CESM in hoofdstuk 3. Eerdere in de literatuur vermelde metingen aangaande de stralingsdosis van CESM lieten waarden zien die varieerden tussen gelijk aan, tot meer dan 50\% hoger ten opzichte van FFDM. We hebben de dosis die door het apparaat werd aangegeven systematisch gevalideerd met behulp van fantoom metingen en vonden dat zowel onze metingen als de metingen die door de unit werden opgegeven overeen kwamen. Vervolgens hebben we aangetoond dat de stralingsdosis van de commercieel verkrijgbare CESM eenheid $81 \%$ hoger was ten opzichte van de in ons instituut gemaakte FFDM. Daarom hebben we aanbevolen de absolute dosiswaarden (in mGy) te gebruiken voor het vergelijken van CESM en FFDM in plaats van relatieve waarden in percentages. Hoewel de stralingsdosis van CESM binnen internationaal aanvaarde limieten blijft, moet stralingsdosis altijd worden gerelateerd aan de klinische vraagstelling en relevantie, vooral bij jonge vrouwen waar MRI een alternatief is.

In hoofdstuk 4 bestudeerden we de beeldkwaliteit van de Low Energy (LE) beelden (die onderdeel zijn van een CESM onderzoek) met FFDM. De LE beelden, afkomstig van verwezen vrouwen uit het bevolkingsonderzoek naar borstkanker, werden vergeleken met hun enkele weken eerder vervaardigde FFDM beelden van het borstkankerscreening onderzoek. Met behulp van de beeldkwaliteit criteria die 
zijn vastgesteld door de 'European Reference Organisation for Quality Assured Breast Screening and Diagnostic Services' (EUREF) hebben we laten zien dat LE beelden niet alleen zijn lage-energie-beelden die gelijk zijn aan FFDM beelden, maar ook dat calcificaties beter werden gevisualiseerd op LE beelden. De afgrenzing van de musculus pectoralis was echter minder accuraat zichtbaar op de LE beelden, maar vermoedelijk zal dit niet tot klinische dilemma's leiden. Aanvullende fantoom metingen werden uitgevoerd om de onderliggende oorzaak van deze observaties te begrijpen. Verschillen werden waarschijnlijk veroorzaakt door verschillen in de post-processing algoritmen omdat er ondanks grondige analyse geen significante verschillen konden worden gedetecteerd. Daarom concludeerden wij dat indien er een indicatie is voor het vervaardigen van een CESM onderzoek, de conventionele mammografie achterwege gelaten kan worden.

In hoofdstuk 5 hebben we een tussentijdse analyse uitgevoerd van de diagnostische prestaties van CESM, in de eerste zes maanden na installatie van de CESM unit, bij 113 patiënten die waren verwezen vanuit het bevolkingsonderzoek voor borstkanker. Slechts drie van de 116 verwezen vrouwen hadden om verschillende redenen geen CESM onderzoek ondergaan. Voor deze populatie waren zowel de sensitiviteit alsook de negatieve voorspellende waarde met gebruik van CESM 100\% (FFDM 96,9\% en 97,1\%, respectievelijk). De belangrijkste stijging werd echter waargenomen voor de specificiteit en de positief voorspellende waarde, waarbij CESM een specificiteit van 87,7\% toonde (FFDM 42,0\%) en een positief voorspellende waarde van 76,2\% (FFDM 39,7\%). Concluderend kunnen we stellen dat CESM niet alleen uitvoerbaar is in een klinische setting, maar ook een veelbelovend 'probleemoplossend hulpmiddel' voor vrouwen die worden verwezen vanuit de borstkankerscreening.

Het evalueren van de reproduceerbaarheid van resultaten in een grotere groep beoordelaars is een belangrijke stap bij de introductie van elke nieuw beeldvormende modaliteit. In hoofdstuk 6 onderzochten we daarom in 199 patiënten de diagnostische accuratesse van CESM, nu bekeken door 10 beoordelaars. Vier beoordelaars waren ervaren CESM gebruikers, drie waren ervaren mamma radiologen zonder voorafgaande CESM ervaring en drie beoordelaars waren radiologen in opleiding met beperkte CESM en mammografie ervaring. De waarden van sensitiviteit, specificiteit, positief voorspellende waarde en de oppervlakte onder de ROCcurve stegen in een trend vergelijkbaar met de resultaten van de tussentijdse analyse van hoofdstuk 5, ongeacht de ervaring die de beoordelaar had met CESM.

We keken verder ook naar het aantal en ook de oorzaken van zowel fout positieve en fout negatieve bevindingen. Van de 199 gevallen werden slechts drie gevallen als fout negatief geïnterpreteerd door meer dan één beoordelaar. Verdere analyse toonde aan dat twee van deze laesies op de rand van het beeld waren afgebeeld. De derde laesie was een mucineus carcinoom, waarvan bekend is dat dit type carcinoom ook op MRI weinig tot geen aankleuring kan laten zien. Deze afwijkingen zijn een potentiële valkuil in de interpretatie van CESM beelden. Daarnaast 
waren fibroadenomen de meest voorkomende fout positieve bevinding op CESM, afwijkingen waarvan we weten dat deze op MRI ook kunnen aankleuring kunnen laten zien.

In hoofdstuk 7 onderzochten we de accuratesse van CESM om de uitgebreidheid van borstkanker te bepalen (met histopathologische resultaten als gouden standaard). Ook vergeleken we de geschatte uitgebreidheid met MRI (indien beschikbaar bij de betreffende patiënt). We vonden dat zowel de correlatie en overeenkomst tussen CESM, histopathologie en MRI goed waren. In de toekomst kan, bij de preoperatieve evaluatie van borstkankerpatiënten, een MRI van de borsten na het uitvoeren van CESM mogelijk achterwege blijven.

In hoofdstuk 8 worden de resultaten van dit proefschrift besproken. Ook wordt een vooruitblik op toekomstige ontwikkelingen gepresenteerd. 


\section{Valorisation}




\section{Social impact}

Breast cancer is one of the leading causes of cancer death among women worldwide. The Dutch breast cancer screening program, established to detect (early stage) breast cancer, has seen a recall rate of $2-3 \%$. Approximately $70 \%$ of these recalls prove to be false positives.

Radiological abnormalities that later prove erroneous engender unnecessary anxiety and needless follow-up testing, which in turn generates unnecessary health care costs. Rapid clarification to establish a final diagnosis is especially important in the work-up of these patients.

\section{Relevance of this thesis}

In this thesis, our aim was to demonstrate that Contrast enhanced spectral mammography (CESM) is feasible and safe in clinical practice. We also assessed image quality and evaluated if this new technique did not come at the expense of unacceptable radiation exposure. Our research therefore focused on women recalled from the breast screening program who underwent CESM as part of their clinical work-up. CESM was found to be superior to conventional digital mammography despite the relatively low disease prevalence in this population. However, breast cancer CESM is not an option because the radiation dose still exceeds that of mammography, and CESM requires intravenous administration of iodine-based contrast agents.

Currently, Dutch women recalled from the screening program are informed by their general practitioner and referred to a breast cancer clinic of their choice for further analysis. Due to existing waiting lists a delay of several days is common before the patient is seen by a nurse practitioner or surgeon, who then refers the patient to the Radiology department for further imaging and/or biopsy. Since recall is based on the detection of a radiological abnormality one might consider changing the approach in order to shorten the diagnostic pathway. This could be achieved through direct referral to an (expert breast-) radiologist who could rapidly discriminate between benign lesions (such as superposition or cyst) and lesions that need further work-up.

As discussed in this thesis, the use of CESM not only allows for more accurate breast cancer detection than conventional mammography, but also for more confident detection of false positive recalls. This could abolish the need for mammographic follow-ups after six, twelve or even eighteen months. In addition, CESM technique has the potential to replace breast MRI in preoperative assessment.

Rapid access and implementation of an 'intermediate stage' between screening and referral to a breast clinic. The results of this thesis advocate such an approach in 
which CESM plays a significant role. This could have several potential benefits: (1) less interval cancers due to the higher diagnostic accuracy of CESM as compared to conventional mammography; (2) reduced patient anxiety due to rapid access and confident diagnosis of false positive recalls; and (3) reduction in health care costs due to the absence of unnecessary downstream testing.

\section{Target groups}

The results of this thesis may be of interest to radiologists, radiology departments, breast care clinics and last but not least, the patients themselves.

When using CESM, diagnostic accuracy will increase regardless of radiologist experience. CESM can therefore be introduced without any significant learning curves. In addition, CESM provides potential downstream benefits such as the avoidance of more time-consuming, invasive or costly tests (e.g. ultrasound, MRI or biopsies). From a technical perspective, most (modern digital) mammography units can be upgraded to include CESM technology; no additional equipment is required (although an automated injector is recommended for standardized intravenous contrast delivery).'

In addition to its use as problem-solving modality for women recalled from the breast screening program, CESM has numerous other potential indications such as inconclusive full-field digital mammography findings, neoadjuvant chemotherapy response monitoring, high risk patient screening, breast-conserving therapy evaluation, and unknown primary tumour evaluation, which are currently all breast MRI indications. Since breast MRI is not widely available in most countries even in Europe, upgrading existing mammography units with CESM could improve the diagnostic imaging chain. Furthermore, CESM might serve as an excellent alternative where contraindications for breast MRI exist (obesity, claustrophobia, metal implants, etc.).

Primary use of CESM in breast care clinics can reduce the number of false positives referred from cancer screening. This would lead to an increased cancer prevalence in the recall population visiting the breast cancer clinics, enabling a focus shift from ruling out suspected malignancy to (true) breast cancers. A CESM-based work-up would also reduce the number of short-term follow-up lesions (so called BI-RADS 3), and accelerate the diagnostic pathway, frequently rendering breast MRI superfluous.

An advantage for patients is the increased specificity of CESM which will reduce the number of recalls, and since CESM is a straightforward imaging method, early access would reduce recall-patient anxiety. CESM was originally developed as an aid in the detection of breast cancer in women with dense breast tissue, and therefore, it could be of great interest for patients with dense breasts. 
Even though CESM is a rather novel modality in the field of breast imaging, studies showing its strong potential and clinical relevance are being published in rapid succession, resulting in an increased number of units being installed worldwide. Over the next few years CESM is expected to become accepted as one of the standard breast imaging modalities, offering immediate solutions for a relevant clinical and social problem. 


\section{Dankwoord}


Wat is een dissertatie zonder dankwoord. Niet alleen zou het niet compleet zijn, voor velen zijn dit waarschijnlijk de eerste pagina's uit deze thesis die daadwerkelijk gelezen worden. Ik heb lang nagedacht wat ik hier op papier moet zetten. Immers, wetenschap komt uit het hoofd en ontwikkeld zich constant verder. Alles wat nu actueel is kan over enkele maanden alweer zijn ingehaald door nieuwe ontwikkelingen. Een dankwoord is echter iets wat uit het hart geschreven wordt en heeft daarom een intrinsieke waarde die onaangetast blijft door het verstrijken van de tijd. Ik vind het daarom ook het moeilijkste deel van dit boekje om op papier te zetten. Een simpel: 'iedereen bedankt' zou zeker niet volstaan. In mijn research naar het schrijven van een dankwoord (jazeker, ook hier kun je research naar doen) blijkt het gebruikelijk in het dankwoord iedereen te bedanken die op enigerlei wijze een bijdrage heeft geleverd aan de totstandkoming van het proefschrift. Dit gelezen hebbend sta ik dan ook voor een onmogelijke opgave. Aangezien het leven van toevalligheden aan elkaar hangt (waarbij het ene gelukkige toeval vaak leidt tot het volgende) heeft alles wat is gebeurd in de jaren voorafgaand aan dit promotietraject geleid tot deze thesis. Ik wil daarom niet alleen diegenen bedanken die direct met dit proefschrift maken hebben maar ook zij die op enigerlei wijze hebben bijgedragen aan de toevalligheden die hebben geleidt tot dit resultaat.

Vaak is geluk een kwestie van op de juiste plaats op de juiste tijd aanwezig te zijn. Dit gelukkig toeval overkwam mij toen ik in 2004 begon aan de opleiding tot radioloog in Maastricht. Mijn toenmalige maar ook huidige collega's zorgen dat ik elke dag weer met plezier aan het werk ga. Daarom wil ik alle collega's danken voor de samenwerking in de afgelopen jaren waardoor de voorwaarden zijn gecreëerd om dit promotietraject tot een goed einde te brengen. Daarnaast ben ik een aantal mensen in het bijzonder dank verschuldigd.

Om te beginnen dank ik professor Boetes, beste Carla. Jouw komst naar Maastricht heeft toentertijd voor een kettingreactie gezorgd in de ontwikkeling van de mammadiagnostiek. In korte tijd was jij erin geslaagd om de mammografie naar een hoger niveau te tillen. Jouw inzichten en jouw kennis waren samen met jouw tomeloze inzet de basis voor de hoge kwaliteit die de mammadiagnostiek op de afdeling nu heeft en daarmee was je ook de initiële aanstichter van dit promotietraject. In korte tijd heb je ook een onuitwisbare indruk achtergelaten op ons allen. Groot was dan ook de verslagenheid op de afdeling toen wij kennis moesten nemen van jouw heengaan. Maar waar je nu ook zou mogen zijn wees gerust dat jouw nalatenschap goede handen is.

Dank aan mijn promotores: professor Wildberger en professor Beets-Tan.

Beste Joachim. Sinds je komst naar Maastricht als hoofd van de afdeling is er veel veranderd. Niet alleen op onze afdeling maar des te meer in de wereld om ons heen. Ik bewonder daarom ook de manier waarop je telkens weer de afdeling door 
deze veranderende tijden weet te navigeren. Het is ook tekenend voor jouw wijze van leidinggeven dat een promotietraject zoals dit mogelijk is. Dank je voor deze kans die je me hebt geboden om te kunnen promoveren. Je inzicht en oog voor detail heeft menig artikel in dit proefschrift verbeterd. In onze gesprekken weet je altijd dingen in het juiste perspectief te plaatsen. Het is daarom ook een voorrecht en genoegen om op een afdeling te werken met jou aan het roer.

Beste Regina. Tijdens mijn fellowship abdominale radiologie heb ik van je geleerd dat de kliniek veeleisend kan zijn maar dat openstaan voor de vragen en eisen van de kliniek op den duur zijn eigen beloningen meebrengt. Jouw mening dat research niet ver van de kliniek af moet staan komt tot uiting in dit proefschrift. Je commentaren hebben ervoor gezorgd dat het ook 'to the point' bleef. Helaas voor ons was het voor jou tijd voor de volgende stap in je carrière. Ik wens je dan ook veel succes als hoofd van de afdeling in het AVL.

Dank aan mijn copromotor dr. Lobbes. Beste Marc. Halverwege de opleiding tot mammaradioloog door Carla wist jij na haar overlijden in sneltreinvaart haar verantwoordelijkheden over te nemen. Door jouw gedrevenheid en ook het enthousiasme waarmee jij nieuwe kansen en nieuwe mogelijkheden aangreep en door de visie die jij deelde met Carla heb jij in de afgelopen jaren de mammadiagnostiek in Maastricht verder geleid naar het huidige niveau. Jouw hulp bij de totstandkoming van dit proefschrift is niet alleen onmisbaar, het hele project was waarschijnlijk onmogelijk geweest. Meer dan eens heb je mij een duwtje in de goede richting gegeven. Ik dank je voor wijze waarop je mij gemotiveerd hebt gehouden in dit traject dat we in 2014 zijn begonnen. Door het introduceren van contrast mammografie op de afdeling ontstond de mogelijkheid om onderzoek te doen waarbij de trein begon te rijden. We hebben allebei denk ik niet beseft dat deze rijdende trein meer als een Japanse hogesnelheidstrein doordendert. Op naar de volgende passagier.

Dank aan de leden van de beoordelingscommissie, bestaande uit Prof. dr. M.F. von Meyenfeldt, Prof. dr. V.C.G. Tjan-Heijnen, Prof. dr. C. Van Ongeval, Prof. dr. W.H. Backes en dr. J. Veltman. Ik wil u danken voor de tijd en aandacht die u aan dit proefschrift heeft geschonken.

Dank aan Ivo Houben. Dank voor je snelle antwoorden en je hulp bij de statistiek. Zelden zo'n rustige jongeman gezien die zo gemotiveerd is, alleen omdat het je leuk lijkt om iets aan research te doen. Ik voorzie een mooie carrière, niet alleen als wetenschapper, maar ook als dokter. Ik hoop in de nabije toekomst nog veel met je te mogen samenwerken.

Dank aan Cécile Jeukens en Leonie Paulis. Jullie wil ik danken, niet alleen voor de bijdragen aan dit proefschrift, maar ook voor een kijkje in de keuken van de klinische fysica. Ik ben erg blij dat jullie complexe zaken zo kunnen uitleggen dat zelfs 
ik het ook kan begrijpen. Dankzij jullie enthousiasme voor dit project hebben we niet alleen de Jan Hendriks prijs in de wacht gesleept, maar ook de basis kunnen leggen voor verdere mooie publicaties. Op een mooie verdere samenwerking en hopelijk nog meer mooie prestaties in de toekomst

Dank aan Patty Nelemans voor je praktische en klinische benadering van statistische hobbels. De sessies die we met jou hebben gehad waarbij we naar methodologie en analyses keken zijn altijd zeer waardevol gebleken. Jouw aanvullende commentaren zorgden ervoor dat we veel gras voor de voeten van reviewers konden wegmaaien en heeft er zeker voor gezorgd dat we papers met hoge kwaliteit konden afleveren.

Verder wil ik, naast de overige medeauteurs van de artikelen in dit proefschrift, in het bijzonder professor Mireille van Goethem, professor Ruud Pijnappel, Susanne Gommers , Caroline Frotscher, Janneke Houwers, Renate Prevos en Wai-Lan Mok danken voor hun bijdrage. Jullie hebben de tijd genomen om in jullie vrije tijd bijna 200 cases te scoren, waarbij een aantal van jullie bereid zijn geweest om naar het verre Maastricht af te reizen.

Dank aan de lieve dames van de mammo: Afsaneh, Betina, Charlotte, Emmely, Eveline, Fia, Hilde, Jenny, Marion, Marlene, Mechtild, Monique, Nancy, Nicky, Odette en Rosan. Wat zou dit proefschrift zijn zonder jullie enthousiaste inzet om elke keer weer de contrast mammografieën te maken. Het is elke keer weer een plezier om met jullie te zorgen voor patiënten want dat is wat jullie zeker op de mammografie leveren, patiëntenzorg. De manier waarop jullie, zonder uitzondering, elke keer weer jullie best doen om elke patiënt gerust te stellen getuigt van inzet en compassie.

Mijn lieve collega's en tevens paranimfen Christianne Hoeberigs en Linda Jacobi wil ik danken voor hun luisterend oor en steun in de voorbereiding voor mijn verdediging. Ik vind het bijzonder fijn dat jullie mij op deze dag willen bijstaan. Wat jullie na de verdediging op het feest voor mij in petto hebben weet ik ten tijde van het schrijven van dit dankwoord niet. Ik zal ongetwijfeld niet gespaard worden. Maar zoals jullie weten, ik zou het ook niet anders willen.

Dank aan mijn overige collega's van de staf radiologie en nucleaire geneeskunde: Frans, Jan, Marco, Susanne, Susanne, Rick, Michiel, Paul, Karin, Janneke, Marinus, Christiaan, Ed, Daan, Felix, Miguel, Simon, Annika, Maud, Sigrid, Stefan, René en Wim. Ik ben voor een deel met en deels door jullie opgeleid. Ik heb geluk dat ik in Maastricht zulke fijne collega's heb.

Ook wil ik de arts-assistenten niet vergeten te danken: Tarik, Camille, Vincent, Rutger, Wouter, Tim, Pauline, Anna, Kemal, Rachelle, Doenja, Ione, Leonie, Monique, Bibi, Karlijn, Casper, Esther, Martijn, Adri, Jochem, Anni, Sanne, Thomas, Vic- 
tor, Nathalie, Bart, Tineke, Andrew en ook alle andere oud assistenten. Sommige van jullie ken ik langer, andere nog maar kort. Ben er nog steeds van overtuigd dat ik net zoveel van jullie leer en heb geleerd als jullie van mij.

Dank aan de dames van het secretariaat van de radiologie: Peggy, Monique, Monique, Elfie en Christianne. Dank voor al het geregel, advies en voor de dropjes die menig staflid op de been houden. Geloof me, het zijn niet alleen de dropjes die ervoor zorgen dat iedereen even bij jullie op het secretariaat blijft hangen, al maken ze het nog gezelliger.

Zoals ik al eerder schreef is het praktisch onmogelijk voor mij om iedereen te bedanken. Een aantal mensen zijn echter de rode draad die door mijn leven loopt. Daarom wil ik hen als laatste noemen in dit dankwoord.

Lieve mama. Jij kwam samen met mij in 1973 naar Nederland om je bij pa te voegen. Jullie zijn uit Suriname vertrokken niet vanwege economische redenen maar omdat volgens de dokters in Suriname het koelere klimaat van de koude Nederlanden beter zou zijn voor mijn gezondheid. Pa is er niet meer maar ik ben jullie eeuwig dankbaar voor de offers die jullie hebben gebracht om jullie kinderen de kans te geven op een goede toekomst.

Lieve Jasmijn, Lynn en Samuel. Mijn prachtige, lieve kinderen. Ook al ging er wel meer dan eens een weekend of uitje verloren omdat ik aan dit proefschrift moest werken, jullie vonden het nooit erg. Ik geniet van de momenten dat ik jullie zie spelen, huiswerk zie maken of gewoon naast jullie op de bank zit. Ik ben blij dat ik voor jullie die 'gekke papa' kan zijn. Jullie zijn het waar ik het allemaal voor doe.

Tot slot mijn lieve, lieve Wendy. Als ik ooit op de juiste plaats op de juiste tijd aanwezig ben geweest is het wel de dag dat wij elkaar voor het eerst zagen. Vanaf het moment dat we elkaar leerden kennen was een half woord van mij voor jou genoeg. Je kent me beter dan ik mezelf ken. Je liefde, steun en gezelschap zijn onmisbaar. Zoals de Beach boys ooit al zongen: 'God only knows what I'd be without you'. Laten we samen genieten van de mooie dingen die verder op ons pad gaan komen. 



\section{Biography}




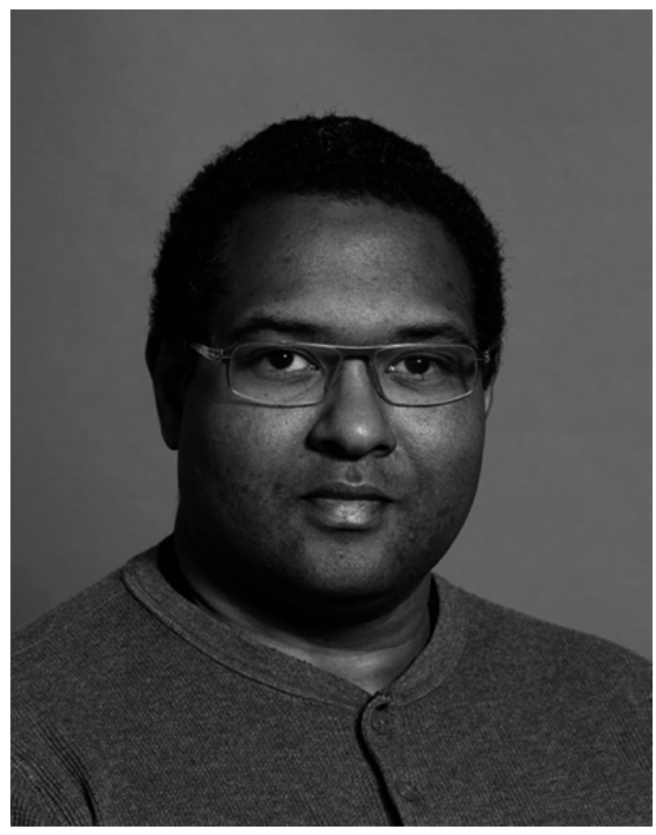

Ulrich Carlos Lalji was born on September 18, 1972, in Paramaribo, Suriname. About a year after his birth he moved with his family to the Netherlands. After graduating from high school (Strabrecht College, Geldrop) he moved to Nijmegen, where he studied medicine at the Radboud University. In 1999 he finished his internships. He started as an emergency physician at the small hospital in the city of Velp, alternating shifts in psychiatry, pulmonology, neurology and internal medicine. In 2001 he started working as a physician in the surgical department of hospital 'de Gelderse Vallei' in the city of Ede until 2004. It was in that same year that he began his residency in radiology at the Maastricht University Medical Centre (MUMC+). In 2009, after completing his residency, he started with his fellowship in abdominal radiology in Maastricht. Nowadays he is working in the MUMC+ as a radiologist specializing in thoracic radiology, mammography, abdominal radiology and non-vascular-oncological interventions. He started his PhD in 2014 under the guidance of his supervisors Prof. Dr. Joachim Wildberger, Prof. Dr. Regina Beets-Tan and his co-promoter Dr. Marc Lobbes.

Ulrich Carlos Lalji werd geboren op 18 september 1972, in Paramaribo, Suriname. Ongeveer een jaar na zijn geboorte verhuisde hij met zijn familie naar Nederland. $\mathrm{Na}$ zijn afstuderen aan de middelbare school (Strabrecht College, Geldrop) verhuisde hij naar Nijmegen, waar hij geneeskunde studeerde aan de Radboud Universiteit. In 1999 behaalde hij het arts examen. Hij begon als een spoedeisende hulp arts in het kleine ziekenhuis van de stad Velp, wisselend diensten draaiend voor psychiatrie, longziekten, neurologie en interne geneeskunde. In 2001 begon hij als arts-assistent te werken op de afdeling chirurgie in ziekenhuis 'de Gelderse Vallei' in Ede tot 2004. In datzelfde jaar begon hij aan zijn radiologie opleiding in Maastricht aan het Maastricht Universitair Medisch Centrum (MUMC+). In 2009, na het afronden van zijn opleiding tot radioloog, doorliep hij een fellowship in de abdominale radiologie in Maastricht. Tegenwoordig is hij werkzaam in het MUMC+ als radioloog gespecialiseerd in thoracale radiologie, mammografie, abdominale radiologie en niet-vasculaire-oncologische interventies. Hij begon zijn promotietraject in 2014 onder leiding van zijn promotores prof. Dr. Joachim Wildberger, prof. Dr. Regina Beets-Tan en zijn copromotor Dr. Marc Lobbes. 



\section{List of publications}




\section{List of publications}

Radiation exposure of digital breast tomosynthesis using an antiscatter grid compared with full-field digital mammography.

Paulis LE, Lobbes MB, Lalji UC, Gelissen N, Bouwman RW, Wildberger JE, Jeukens CR. Invest Radiol. 2015 Oct;50(10):679-85.

CT-Guided Percutaneous Transthoracic Needle Biopsies Using 10G LargeCore Needles: Initial Experience.

Lalji UC, Wildberger JE, Zur Hausen A, Bendek M, Dingemans AM, Hochstenbag M, Das M. Cardiovasc Intervent Radiol. 2015 Dec;38(6):1603-10.

Evaluation of low-energy contrast-enhanced spectral mammography images by comparing them to full-field digital mammography using EUREF image quality criteria.

Lalji UC, Jeukens CR, Houben I, Nelemans PJ, van Engen RE, van Wylick E, Beets-Tan RG, Wildberger JE, Paulis LE, Lobbes MB. Eur Radiol. 2015 Oct;25(10):2813-20.

The quality of tumor size assessment by contrast-enhanced spectral mammography and the benefit of additional breast MRI.

Lobbes MB, Lalji UC, Nelemans PJ, Houben I, Smidt ML, Heuts E, de Vries B, Wildberger JE, Beets-Tan RG. J Cancer. 2015 Jan 5;6(2):144-50.

Contrast-enhanced dual-energy mammography: a promising new imaging tool in breast cancer detection.

Lalji U, Lobbes M. Womens Health (Lond Engl). 2014 May;10(3):289-98.

Value of gadofosveset-enhanced MRI and multiplanar reformatting for selecting good responders after chemoradiation for rectal cancer.

Heijnen LA, Maas M, Lahaye MJ, Lalji U, Lambregts DM, Martens MH, Riedl RG, Beets GL, Beets-Tan RG.Eur Radiol. 2014 Aug;24(8):1845-52.

Radiation exposure of contrast-enhanced spectral mammography compared with full-field digital mammography.

Jeukens CR, Lalji UC, Meijer E, Bakija B, Theunissen R, Wildberger JE, Lobbes MB. Invest Radiol. 2014 Oct;49(10):659-65.

Contrast-enhanced spectral mammography in patients referred from the breast cancer screening programme.

Lobbes MB, Lalji U, Houwers J, Nijssen EC, Nelemans PJ, van Roozendaal L, Smidt ML, Heuts E, Wildberger JE. Eur Radiol. 2014 Jul;24(7):1668-76.

A pulmonary abscess, beware of lung cancer!

Hendriks LEL, Hochstenbag MMH, Lalji UC, Dingemans AMC. Respiratory Medicine CME (2011) vol. 4 (4) p. 157-159. 


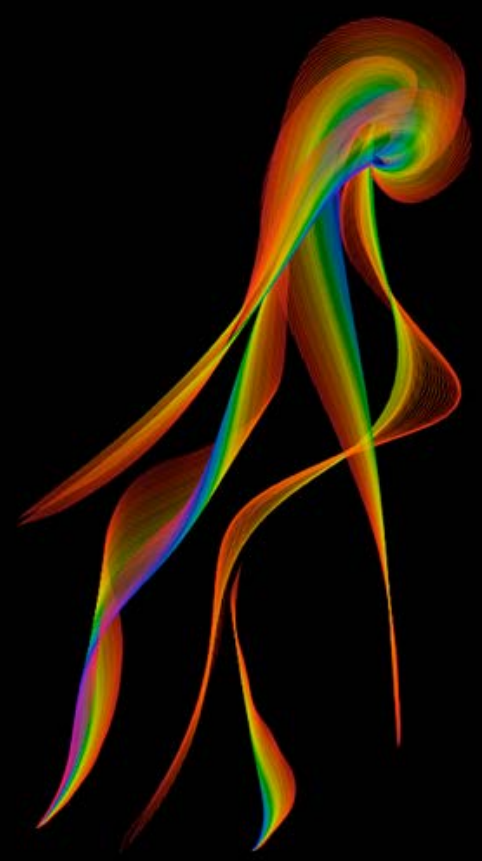

